\title{
WestVirginiaUniversity
}

THE RESEARCH REPOSITORY @ WVU

Graduate Theses, Dissertations, and Problem Reports

2014

\section{A Multi-Agent Design for Power Distribution Systems Automation}

M. Jawad Ghorbani

West Virginia University

Follow this and additional works at: https://researchrepository.wvu.edu/etd

\section{Recommended Citation}

Ghorbani, M. Jawad, "A Multi-Agent Design for Power Distribution Systems Automation" (2014). Graduate Theses, Dissertations, and Problem Reports. 120.

https://researchrepository.wvu.edu/etd/120

This Dissertation is protected by copyright and/or related rights. It has been brought to you by the The Research Repository @ WVU with permission from the rights-holder(s). You are free to use this Dissertation in any way that is permitted by the copyright and related rights legislation that applies to your use. For other uses you must obtain permission from the rights-holder(s) directly, unless additional rights are indicated by a Creative Commons license in the record and/ or on the work itself. This Dissertation has been accepted for inclusion in WVU Graduate Theses, Dissertations, and Problem Reports collection by an authorized administrator of The Research Repository @ WVU.

For more information, please contact researchrepository@mail.wvu.edu. 


\title{
A Multi-Agent Design for Power Distribution Systems Automation
}

\author{
M. Jawad Ghorbani
}

Dissertation submitted to the

Benjamin M. Statler College of Engineering and Mineral Resources at West Virginia University

in partial fulfillment of the requirements for the degree of

Doctor of Philosophy in Electrical Engineering

\author{
Ali Feliachi, Ph.D. \\ Powsiri Klinkhachorn, Ph.D. \\ Yaser P. Fallah, Ph.D. \\ Hong-Jian Lai, Ph.D. \\ M. A. Choudhry, Ph.D., Chair
}

Lane Department of Computer Science and Electrical Engineering

Morgantown, West Virginia

2014

Keywords: [Multi-Agent System, Fault Location and Isolation, Restoration, Reinforcement Learning, Load Management and Forecast, Hardware Implementation] 


\section{ABSTRACT}

\section{A Multi-Agent Design for Power Distribution Systems Automation}

\section{Jawad Ghorbani}

A new Multi Agent System (MAS) design for fault location, isolation and restoration in power distribution systems is presented. In proposed approach, when there is a fault in the Power Distribution System (PDS), MAS quickly isolates the fault and restores the service to fault-free zones. Hierarchical coordination strategy is introduced to manage the agents which integrate the advantages of both centralized and decentralized coordination strategies. In this framework, Zone Agent (ZA) locate and isolate the fault based on the locally available information and assist the Feeder Agent (FA) for reconfiguration and restoration. FA can solve the restoration problem using the existing algorithms for the $0-1$ Knapsack problem.

A novel Q-learning mechanism is also introduced to support the FAs in decision making for restoration. Also a distributed MAS-Based Load Shedding (LS) technique has been used to supply as many of higher priority customers as possible, in case there is more demand than generation. The design is illustrated by the use of simulation case studies for fault location, isolation and restoration on West Virginia Super Circuit (WVSC) and hardware implementation for fault location and isolation in a laboratory platform. The results from the case studies indicate the performance of proposed MAS designs. 
"I've missed more than 9000 shots in my career. I've lost almost 300 games. 26 times I've been trusted to take the game winning shot and missed. I've failed over and over and over again in my life. And that is why I succeed."

Michael Jordan 


\section{Acknowledgements}

Firstly, I would like to thank my PhD committee chair and my supervisor Dr. Muhammad A. Choudhry. It was a blessing to be one of his $\mathrm{PhD}$ students and be able to conduct research with him. I would also like to thank Dr. Feliachi for his constructive comments.

I would like to thank Dr. Powsiri Klinkhachorn, Dr. Yaser P. Fallah and Dr. HongJian Lai for their invaluable input to my research and for being in my committee. Their guidance helped me immensely to shape and improve my research as well as its presentation in this document. I would also like to present my sincere thanks to West Virginia University and its staff for providing all the wonderful facilities and services, which helped me on a day to day basis during my research as well as during my daily life in the beautiful city of Morgantown.

Finally, I would like to thank my mother, my father, who supported me all throughout my life. Whenever I needed their support, they were always there for me with their unconditional love. 


\section{Contents}

Abstract

$\begin{array}{ll}\text { Acknowledgements } & \text { iii }\end{array}$

Contents

List of Figures $\quad$ v

List of Tables vi vi vis

Abbreviations vii

Symbols viii

1 Introduction $\quad 1$

1.1 Problem Statement . . . . . . . . . . . . . . . . . . 2

1.1.1 Reliability Issues . . . . . . . . . . . . . . . . . . . . . . . . . . . . . . . . .

1.2 Statement of This Dissertation . . . . . . . . . . . . . . . 4

1.2 .1 Contributions . . . . . . . . . . . . . . . 6

1.3 Structure of This Dissertation . . . . . . . . . . . . . . 7

1.3.1 Dissertation Outline . . . . . . . . . . . . . . 8

1.4 Publications . . . . . . . . . . . . . . . . . 9

2 Literature Review 10

2.1 Multi-Agent Systems . . . . . . . . . . . . . . . . . . . 10

2.2 Fault Location \& Isolation Methods . . . . . . . . . . . . . . . . . . 12

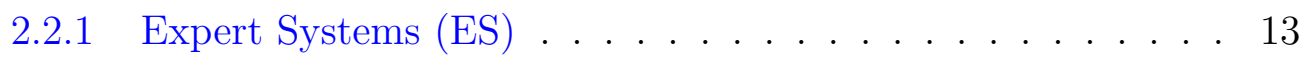

2.2.2 Artificial Neural Networks (ANNs) . . . . . . . . . . 13

2.2 .3 Petri-Nets $(\mathrm{PN}) \ldots \ldots \ldots \ldots . \ldots \ldots$

2.3 Service Restoration . . . . . . . . . . . . . . . . . . . . 14

2.3.1 Heuristic Techniques \& Knowledge Based Systems . . . . . . 15

2.3.2 Mathematical Programming . . . . . . . . . . . 16

2.4 MAS Communication . . . . . . . . . . . . . . . . 17 
2.5 Learning Algorithms . . . . . . . . . . . . . . . . . . . 18

2.6 Load Shedding . . . . . . . . . . . . . . . . . . . . . . 19

2.7 Available FLIR Systems . . . . . . . . . . . . . . . . . . . 21

2.7.1 Intelliteam SG $[1] \ldots \ldots \ldots . \ldots . \ldots . \ldots 22$

2.7.1.1 IntelliTEAM Theory of Operation . . . . . . . 22

2.7.1.2 FLIR \& IntelliTEAM . . . . . . . . . . . 24

3 Multi Agent Systems $\quad 26$

3.1 MAS Concept . . . . . . . . . . . . . . . . 26

3.1 .1 Agent Definition . . . . . . . . . . . . . 26

3.1 .2 Intelligent Agent . . . . . . . . . . . . . . . . . 27

3.1.3 Definition of a Multi-Agent System . . . . . . . . . . 27

3.2 MAS Architecture . . . . . . . . . . . . . . . . . . . 28

3.2 .1 Centralized . . . . . . . . . . . . . . . . 28

3.2 .2 Decentralized . . . . . . . . . . . . . 30

3.2 .3 Hierarchical . . . . . . . . . . . . . . . . . . 31

3.2 .4 Hybrid . . . . . . . . . . . . . . . . . 31

3.3 Proposed Architecture . . . . . . . . . . . . . . . . . . . 32

3.3 .1 Agents ......................... 34

3.3.1.1 Zone Agent $(\mathrm{ZA}) \ldots \ldots . \ldots . \ldots 34$

3.3.1.2 Feeder Agent $(\mathrm{FA}) \ldots \ldots . \ldots . . \ldots 34$

3.3.1.3 Substation Agent $(\mathrm{SA}) \ldots \ldots . \ldots . \ldots 35$

3.4 MAS Communications .................... 35

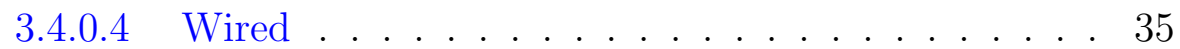

3.4.0.5 Wireless . . . . . . . . . . . . 35

3.4.0.6 Communication Languages . . . . . . . . . 36

4 Fault Location \& Isolation $\quad 38$

4.1 Fault Location Equations . . . . . . . . . . . . . . . . . . . . . 39

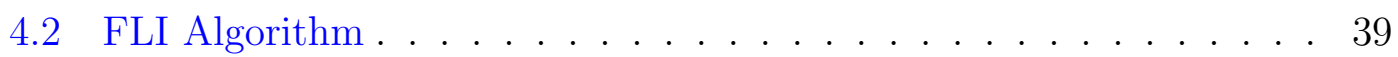

4.3 Fault Classification . . . . . . . . . . . . . . . . . . . . . . . . . . . . . . . 41

4.4 Fault Location Results . . . . . . . . . . . . . . . . . . . . . 42

4.4 .1 Impedance Analysis . . . . . . . . . . . . . . . . . . . 42

4.4.1.1 Case I: phase to ground fault between switch 2 and 342

4.4.1.2 Case II: phase to phase fault between switch 2 and 343

4.4 .2 Proposed Algorithm . . . . . . . . . . . . . . 44

5 Service Restoration $\quad 48$

5.1 Restoration Formulation . . . . . . . . . . . . . . . . . . 49

5.2 Illustration . . . . . . . . . . . . . . . . . . 50

5.3 FLIR Flowchart . . . . . . . . . . . . . . . . . . 51

6 Learning Methodology $\quad 54$

6.1 Motivation . . . . . . . . . . . . . . . . 54

6.2 Reinforcement Learning (Q-Learning) . . . . . . . . . . . . . . 54 
6.2 .1 Learning . . . . . . . . . . . . . . . . . 56

6.2 .2 Exploration ................... 56

6.3 Restoration Learning Approach . . . . . . . . . . . . . . . . 57

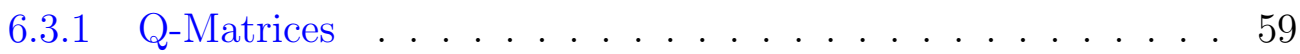

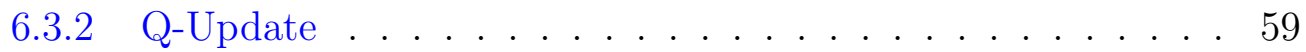

6.3.3 Action Selection Strategy . . . . . . . . . . . 60

7 Restoration Case Studies and Simulations $\quad \mathbf{6 1}$

7.1 West Virginia Super Circuit . . . . . . . . . . . . . . . . . . 61

7.1 .1 Recloser Setting . . . . . . . . . . . . . . . . 62

7.2 Simulation Models . . . . . . . . . . . . . . . . 63

7.2 .1 Power System Model . . . . . . . . . . . . . . . . . . . 63

7.2 .2 MAS Model . . . . . . . . . . . . . . . . . 63

7.3 Restoration Results . . . . . . . . . . . . . . . . . 65

7.3 .1 Case I . . . . . . . . . . . . . . . . . . . 65

7.3 .2 Case II . . . . . . . . . . . . . . . . . . 67

7.4 Performance Analysis . . . . . . . . . . . . . . . . . . . . . 68

8 Communication Performance Analysis $\quad \mathbf{7 0}$

8.1 Latency and Reliability Formulations . . . . . . . . . . . . . . . . . 70

8.1 .1 Latency . . . . . . . . . . . . . . . . . . 71

8.1 .2 Bandwidth......................... 73

8.1 .3 Reliablility . . . . . . . . . . . . . . 73

8.2 Co-simulation of Power System and Communication Network . . . . 74

9 Load Shedding $\quad 78$

9.1 Multi-Agent System ． . . . . . . . . . . . . . . . . . . . 79

9.1.1 Agent Architecture . . . . . . . . . . . . . . . . . 79

9.1.2 Agent Application Design . . . . . . . . . . . . . . . 81

9.2 Active Learning Method . . . . . . . . . . . . . . . . . . . . . 81

9.2 .1 Ink Drop Spread (IDS) . . . . . . . . . . . . . . . 82

9.2.2 Combination of Fuzzy Rules . . . . . . . . . . . . . . . 83

9.3 ALM-Based Load Prediction . . . . . . . . . . . . . . . . . . . . . 84

9.4 Load Shedding Optimization . . . . . . . . . . . . . . . . . . 86

9.4.1 LS algorithm flow . . . . . . . . . . . . . . 86

9.4 LS Formulations . . . . . . . . . . . . . . 86

9.5 Case Studies . . . . . . . . . . . . . . . . . . . . . . 89

9.5.1 Test System . . . . . . . . . . . . . . . . . 90

9.5.2 Case I: Load Shedding Restoration . . . . . . . . . . . . . . 90

9.5 .3 Case II: Including DG's . . . . . . . . . . . . . . . 91

10 Hardware Implementation $\quad 93$

10.1 Multi-Agent Based Fault Location . . . . . . . . . . . . . . . . . 95

10.1.1 MAS Architecture . . . . . . . . . . . . . . 95

10.1.2 Fault Location Equations . . . . . . . . . . . . . 96 
10.1.3 Fault Location and Isolation Algorithm . . . . . . . . . . . 96

10.2 MAS Application Design . . . . . . . . . . . . . . . . . . . 98

10.2.1 Zone Agent Responsibilities . . . . . . . . . . . . . . . . . . . 98

10.2.2 Interaction Between Agents . . . . . . . . . . . . . . . . 99

10.2.2.1 Ontology . . . . . . . . . . . . . . 99

10.2.2.2 Communication . . . . . . . . . . . . 100

10.3 Implementation of Proposed FLI System . . . . . . . . . . . . . . 100

10.3.1 Components . . . . . . . . . . . . . . . . 101

10.3.1.1 SEL . . . . . . . . . . . . . . . . . . 101

10.3.1.2 Processing Units . . . . . . . . . . . . . . . . . . . . . . . . . . . . . . . . .

10.3.2 Communication Media . . . . . . . . . . . . . . 103

10.3.3 Software Program . . . . . . . . . . . . . . . . . . . 103

10.3.4 Results and Discussion . . . . . . . . . . . . . . . . 104

11 Conclusions and Future Work 107

11.1 Conclusions . . . . . . . . . . . . . . . . . . . 107

11.2 Future Work . . . . . . . . . . . . . . . . . . . 110 


\section{List of Figures}

1.1 Comparison of Reliability improvement DUE TO IMPleMENTATION OF FLIR VERSUS CONVENTIONAL RESTORATION [2]

1.2 Finite State machine for MAS . . . . . . . . . . 8

2.1 An example of IntelliteAM used to AUtomate Four OPENLOOP DISTRIBUTION CIRCUITS . . . . . . . . . . . . 23

3.1 Centralized Architecture . . . . . . . . . . . . . . 29

3.2 Hierarchical Architecture . . . . . . . . . . . . . . . . 29

3.3 Distributed and Hybrid Architectures . . . . . . . . . . . . . 30

3.4 West Virginia Super Circuit . . . . . . . . . . . . . . . 33

3.5 MAS Structure . . . . . . . . . . . . . . . . . 33

3.6 ZA And FA Structures . . . . . . . . . . . . . . 34

3.7 Agent interaction Diagram SHOWing the PROTOCOL FOR QUERY-REF COMMUNICATIVE ACT . . . . . . . . . . . 37

4.1 Fault location, isolation algorithm flowChart . . . . . 40

4.2 Recloser operation . . . . . . . . . . . . . . . . . . . 42

4.3 Current Changes For SWitch 1 During AND Before Fault (PER UNIT) . . . . . . . . . . . . . . . . . . . 43

4.4 IMPEDANCE CHANGES FOR SWITCH 1 DURING AND BEFORE FAULT (Per UntT) . . . . . . . . . . . . . . . . . . 43

4.5 Current changes for SWitch 1 During and Before Fault (PER UNIT) . . . . . . . . . . . . . . . . . . . . 44

4.6 IMPEDANCE CHANGES FOR SWITCH 1 DURING AND BEFORE FAULT (PER UNIT) . . . . . . . . . . . . . . . . . . . 44

4.7 Calculated index for DG Penetration of $0 \% \ldots \ldots$. . . . . 46

4.8 Calculated index for DG Penetration of $50 \% \ldots \ldots 7$

4.9 Calculated index for DG penetration of $50 \%$. . . . . 47

5.1 Flowchart of Self-healing FLIR With LEARning of PDS . 53

6.1 LeArning Modules in A FA . . . . . . . . . . . . . 57

6.2 (A) Online And (B) Offline Learning Modes . . . . . . 58

7.1 The WVSC PoWer SYSTEM . . . . . . . . . . . . . 62

7.2 Power distribution system Simulink model . . . . . . . 64 
7.3 Multi agent System model in MATLAB Simulink . . . . . . 64

7.4 Case I and Case II Reconfigurations . . . . . . . . . . . . 68

8.1 Communichtions Between Agents for FliR . . . . . . . . 71

8.2 Series ANd PARALlel COMMUNiCATiOns IN EACH ARCHiteCture 76

8.3 Measurement Data $(V \& I)$ and Transmitted Data to Control Center for Distributed Architecture of MAS 77

9.1 MAS Architecture ....................... 80

9.2 IDS Plane: (A) Five DATA POints SPREAd on A Plane (B) INK SPREADS AND NARROW PATH . . . . . . . . . . . 82

9.3 EACH DATA POINT HAS A PYRAMIDAl FUZZY MEMBERSHIP . . . . 83

9.4 ALM SPlits A MiSO SYSTEM INTO SEVERAL SISO SYSTEMS, EACH SISO SYSTEM IS MODELED BY AN IDS PLANE . . . . . 85

9.5 IDS PLANE FOR SEASONAL EFFECT ON POWER CONSUMPTION [3] 85

9.6 Restoration Flowchart . . . . . . . . . . . . . . . . . . . . . 87

9.7 West Virginia Super Circuit . . . . . . . . . . . . . . . . . . 91

9.8 Voltage Simulation Result for Case I . . . . . . . . . . . . . . 91

9.9 Voltage Simulation Result for Case II . . . . . . . . . . . . 92

10.1 MAS Architecture . . . . . . . . . . . . . . . . . . . . 95

10.2 Zone Agent $(\mathrm{ZA}) \ldots \ldots . \ldots . \ldots 96$

10.3 FAult location, isolation Algorithm FlowChaRT . . . . . . 97

10.4 Agents Communichtion for FLI . . . . . . . . . . . . . . 100

10.5 Agent Implementation . . . . . . . . . . . . . . . . . . . . . . . . . . . . . . . . . . . . . . . . . .

10.6 Implemented PDS Network . . . . . . . . . . . . . . . 105

10.7 LABORATORY IMPLEMENTATIONS . . . . . . . . . . . . . . . . . . . . . . . . 106

10.8 Measured Fault Current and Recloser Operation . . . . . 106 


\section{List of Tables}

3.1 FiPA-ACL Message Structure . . . . . . . . . . . . 36

4.1 Fault location \& Isolation Simulation Results . . . . . . 45

7.1 WVSC Feeders Peak Load Data . . . . . . . . . . . . . 62

7.2 Recloser Setting . . . . . . . . . . . . . . . 63

7.3 Proposed MAS FLIR procedure (CASE I) . . . . . . . . . . . 66

7.4 Number of Communication Messages . . . . . . . . . . . . 68

8.1 Time Delays for latency Calculations . . . . . . . . . . . 72

8.2 Expected Delay for Each Communication Link . . . . . . . 75

8.3 Best and Worst Case Latency for MAS Structures With Wired and Wireless Communication Media (MS) . . . . . 75

9.1 Agent's Facts . . . . . . . . . . . . . . . . . . 81

9.2 VARIABles USEd in LOAD PREDiCTION MODELS . . . . . . . . . 84

10.1 Facts in the Implemented MAS . . . . . . . . . . . . . . . 99

10.2 Specification of SEL 735 Power Quality Meters . . . . . 102

10.3 SEL-735 Command Summary . . . . . . . . . . . . . . 102

10.4 Specification of Nuvo-1003S . . . . . . . . . . . . . . 103

10.5 Communication Ports Settings . . . . . . . . . . . 103 


\section{Abbreviations}

$\begin{array}{ll}\text { PDS } & \text { Power Distribution System } \\ \text { DSM } & \text { Demand Side Management } \\ \text { FLIR } & \text { Fault Location, Isolation \& Restoration } \\ \text { FLI } & \text { Fault Location, Isolation } \\ \text { LS } & \text { Load Shedding } \\ \text { MAS } & \text { Multi Agent System } \\ \text { SA } & \text { Substation Agent } \\ \text { FA } & \text { Feeder Agent } \\ \text { ZA } & \text { Zone Agent }\end{array}$

FSM Finite State Machine

SAIDI System Average Interruption Duration Index

SAIFI System Average Interruption Frequency Index

CAIDI Customer Average Interruption Duration Index

ACL Agent Communication Language

DA Distribution Automation

DG Distributed Generation

AI Artificial Intelligence

SPS Shipboard Power Systems

NP Nonlinear Programming

GSM Groupe Special Mobile

GPRS General Packet Radio Service

WiMax Worldwide interoperability for Microwave Access

WLAN Wireless Local Area Network

EPOCHS Electric POwer and Communication syncHronizing Simulator 


\section{Symbols}

$\begin{array}{lll}V & \text { voltage } & \mathrm{V} \text { (Volt) } \\ I & \text { current } & \mathrm{A} \text { (Ampere) } \\ P & \text { active power } & \mathrm{W}\left(\mathrm{Js}^{-1}\right) \\ Q & \text { reactive power } & \mathrm{VAR} \\ t & \text { time } & \mathrm{s} \text { (second) } \\ \rho & \text { load priority } & \\ a & \text { action } & \\ s & \text { state } & \\ \beta & \text { learning rate } & \\ R & \text { reward function } & \\ & & \\ \omega & \text { angular frequency } & \operatorname{rads}^{-1} \\ \psi & \text { phase } & \operatorname{rads}^{2}\end{array}$


In the Name of God... 


\section{Chapter 1}

\section{Introduction}

The term 'smart grids' means many things to many people and there is certainly no agreed definition. It is common to hear people say they are working on smart grids anywhere from designing meters or power electronic converters, WiMAX communications to some new look at system dynamics. There is even the debate over whether we should use the term at all. In this work we take the view that the discipline of 'smart grids' has meaning as an area focused on embedded intelligence from a systems approach somewhat ahead of the current state in industry (so may be 'smarter grids'), but it remains to be more clearly defined what we are talking about and what the research goals are, i.e. to put some serious science into the idea. We take the view that this paradigm can apply anywhere in the whole energy network, but the current emphasis is on distribution systems, because that is where the development of automation has been least advanced. Some ideas towards this aim are presented here. Ultimately, at the core of the smart grid agenda, the goal is to achieve a system which is more adaptive and resilient to changing power supply/demand, failures and attacks. The presentation of a clearer formulation inevitably leads to an agenda of estimation, optimization, learning and control. Thus we can conclude that there should be a very exciting road ahead for collaboration between the power and control areas.

In the recent years, the concept of Smart Grids (SG) was proposed [4]. The National Energy Technology Laboratory (NETL) in the United States has presented seven principle characteristics of a smart grid. A smart grid should be able to heal itself after a power system event; it should enable active customer participation, resist attacks, provide power quality for the 21st century needs, accommodate 
all generation and storage options, enable markets, and optimize asset utilization and operate efficiently. Among them, self-heal is the key characteristic. Some literatures also called a smart grid as a self-healing grid [5].

European Technology Platform SmartGrids defined the word "self-healing" as not only automated network restoration strategies that take into account the impact of high penetration of distributed generation and demand side participation, but also high level decentralized preventive control methodologies that will address options for the management of unplanned outages [6]. "Self-healing " was also interpreted as an engineering design that enables the problematic element of a system to be isolated and, ideally, restored to normal operation with little or no human intervention.

\subsection{Problem Statement}

Based on U.S. Department of Energy (DOE) studies, more than $80 \%$ of outages in power systems are directly related to distribution systems. For most utilities, three main outage causes and their average proportion consist of: tree-related failures (29\%), animal related failures (18\%), lightning failures (11\%)[7]. Even though technologies can be employed to mitigate future possible faults, most system outages and faults are inevitable. When a fault occurs in the Power Distribution System (PDS), corresponding reclosers will lock out and the fault-free areas will also lose power. It is essential for the electric utility companies to restore loads in the out-of-service areas as soon as possible. In present power distribution systems, the majority of the operation for power restoration are performed manually. An automated PDS can perform the restoration in less time while providing the benefits of reduction in the number of required crew members.

A self-healing PDS, when subject to a contingency (fault), is able to automatically perform remedial actions to restore the system to the best possible state. The objective of self- healing PDS is to minimize the duration of outages as well as the customers interruptions, in favor of overall PDS reliability. Customer based distribution reliability indices, specially Customer Average Interruption Duration Index (CAIDI) and system average interruption duration index (SAIDI) will be potentially improved. 


\subsubsection{Reliability Issues}

Several cascading failures during the past 40 years spotlighted our need to understand the complex phenomena associated with power network systems and the development of emergency controls and restoration. Widespread outages and huge price spikes during the past few years raised public concern about grid reliability at the national level. According to data from the North American Electric Reliability Council (NERC) and analyses from the Electric Power Research Institute (EPRI), average outages from 1984 to the present have affected nearly 700,000 customers per event annually [8]. Smaller outages occur much more frequently and affect tens to hundreds of thousands of customers every few weeks or months, while larger outages occur every two to nine years and affect millions. Much larger outages affect seven million or more customers per event each decade. These analyses are based on data collected for the US Department of Energy (DOE), which requires electric utilities to report system emergencies that include electric service interruptions, voltage reductions, acts of sabotage, unusual occurrences that can affect the reliability of bulk power delivery systems, and fuel problems.

Fig.1.1 shows the advantages of implementing Fault Location \& Isolation (FLIR) versus conventional operation for a typical distribution feeder. When conventional operation (without FLIR) is used, there is a need for investigating the specific fault location and conducting manual switching to isolate the faulted area and restore service to customers located on healthy feeder sections. In this case customer trouble calls may play an important role, and human intervention, either for fault location or switching operations to restore service, is vital. FLIR on the other side allows detecting faults and restoring affected customers faster and with limited human intervention. When FLIR is used, power is quickly restored to customers located on healthy sections of a feeder. Moreover, the faulted area is delimited by the FLIR scheme, this reduces the time required for fault investigation and patrolling. Moreover, if FLIR switching and protective devices are monitored in real-time then there is no need to wait for customer trouble calls to dispatch crews. Therefore, besides its obvious reliability benefits FLIR also has a direct impact on reducing operators and crews' workload, which increases efficiency and reduces operation costs. 


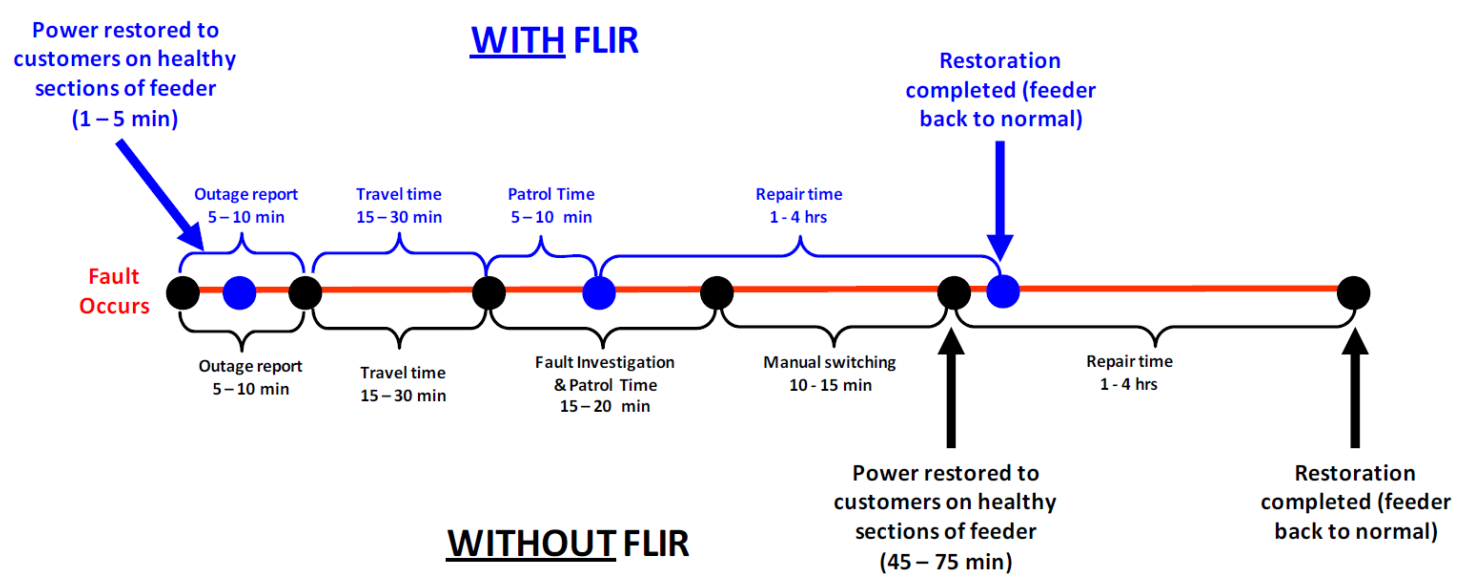

Figure 1.1: Comparison OF REliability improvement duE to implementation of FLIR VERSUS CONVENTIONAL RESTORATION [2]

\subsection{Statement of This Dissertation}

SELF-HEALING of power delivery systems is a concept that enables the identification and isolation of faulted system components and the restoration of service to customers supplied by healthy elements. This activity may be conducted with little or no human intervention and has the objective of minimizing interruptions of service and avoiding further deterioration of system reliability. Self-healing of power distribution systems is conducted via Distribution Automation (DA), specifically through smart protective and switching devices that minimize the number of interrupted customers during contingency conditions by automatically isolating faulted components and transferring customers to an optional source when their normal supply has been lost. Optional sources may include neighbor feeders and Distributed Energy Resources (DER) such as Distributed Generation (DG) and Distributed Energy Storage (DES). For this reason, some authors prefer to use the term "self-restoration" instead of self-healing. It is worth noting that the implementation of self-healing in distribution systems requires schemes that are flexible enough to adapt to changing system loading and configuration conditions (including automatically modify protection settings) and operate distribution system components within their ratings.

Distribution Automation (DA) is a set of technologies that enable an electric utility to remotely monitor, coordinate, and operate distribution components in a realtime mode from remote locations. DA includes substation, feeder and customer automation. DA is a vital component for achieving the selfhealing capabilities, high reliability and power quality of the smart grid, as well as for allowing the 
integration of DER. One of the most popular DA applications is Fault Location, Isolation and Service Restoration (FLIR).

A self-healing restoration method requires a framework for determining the series of control actions during the healing mode of a PDS. Basically, PDS is in the normal state while system parameters are within normal operating range. System enters the abnormal state when an outage happens. After fault location, isolation and restoration, the system goes to temporary restoration state until the fault is cleared. Fast and efficient power restoration in a self-healing PDS entails an automated system design for fault location, isolation, reconfiguration and restoration. In this dissertation various aspects of a self-healing PDS are discussed, including:

- Fault Location: A key aspect of self-restoration when applied to distribution systems is the need to identify fault locations and if possible anticipate fault occurrence.

when a fault occurs in the PDS, the self-healing system is triggered by an automatic re-closer trip in real-time operation, and starts to detect the fault location and identifies the fault type by monitoring data along the feeder.

- Isolation: the first corrective action after locating the fault is to open the relevant switches (in arbitrary order) in order to isolate the fault from the rest of the system.

- Reconfiguration \& Restoration: finding a reconfiguration strategy which determines the switching sequence necessary to reconfigure the system in order to allow the broadest possible power access.

There is a growing trend in the industry for implementing FLIR as well as other DA schemes. This is due to several reasons such as the access to incentives provided through government-funded programs, the maturity of DA technologies and the availability of a variety of communication technologies that facilitate its implementation. FLIR benefits include[2]:

\section{Functional benefits:}

1. Improve SAIDI, SAIFI, and other reliability statistics

2. Reduce "energy not supplied" (kWh) 
3. Provide "premium quality" service

4. Reduce fault investigation time

\section{Monetary benefits:}

1. Increase revenue (sell more energy)

2. Reduce customer cost of outage

3. Additional revenue from "premium quality" customers

4. Labor/vehicle savings

5. Achieve regulatory incentives (when available)

\subsubsection{Contributions}

The main contributions of this work can be summarized as follows.

1. MAS architecture: A hierarchical fully distributed MAS that performs the FLIR using less communicated messages than the existing methods. The framework is introduced in Chapter 3.

2. Real-Time MAS: MAS and power system are simulated to work in realtime and without any interface for synchronizing two different models, which makes the simulation results more accurate and reliable.

3. Fault Location and Isolation Algorithm: Proposed FLI algorithm is fully distributed and ZAs along the feeder can locate and isolate the faulty section by communicating with their immediate neighbors. The FLI algorithm is described in Chapter 4.

4. Restoration Learning algorithm: A reinforcement learning algorithm is developed in conjunction with restoration mathematical programming to take the advantage of restoration experiences and use the learning knowledge for future restorations. Restoration problem formulation and learning approach are presented in Chapters 5 and 6.

5. Distributed Load Shedding: Presented load shedding algorithm in Chapter 9 is a distributed MAS-based method that finds the near-optimal configuration for restoration using the available local data, while shedding least possible number of low priority loads. 
6. Hardware Implementation: Chapter 10 presents the development of a platform compromising of meters, agent components, loads integrated with a power system platform to verify applicability of proposed method in locating and isolating the fault using a real world case study.

\subsection{Structure of This Dissertation}

In this work, authors compare the possible MAS frameworks considering the reliability and communications requirements and proposed a hierarchically decentralized MAS architecture composed of upper and lower level agents [9]. In the bottom-up order, agents are classified as Zone Agents (ZA), Feeder Agents (FA) and Substation Agents (SA). In the proposed framework, ZAs are responsible for locating the fault and isolating it. While reconfiguration and restoration are handled by FAs.

In that framework, ZAs have access to the voltage and current phasor data of themselves and their immediate neighbors to a defined neighborhood through communication. Using these data and by real-time monitoring of indices introduced in this work, ZAs can decide whether the fault is in their neighborhood or not. Following fault location and isolation by ZAs and informing the corresponding FA about the fault information, FA first restores the upstream zones of faulted area and starts negotiating with alternative FAs, that have tie switches to faulty feeder. FAs evaluate the possible reconfiguration and choose the best reconfiguration using deterministic optimization problem solving techniques to supply as many loads as possible.

Fig.1.2 shows the Finite State Machine (FSM) for fault detection isolation and finally restoration processes. According to this FSM after the recloser locks out, agents start their negotiations to locate and isolate the fault. Agents can just communicate with their lower level agents and in normal situations lower level agents should not take any action before consulting with their higher level agents. But in the case of loss of communications with higher level agents, the lower level agents can make their decisions to isolate the fault and restore the power.

Although agents in MAS can solve the optimization techniques to find the near optimal configuration, it is also necessary that they learn new behaviors online, such that the performance of the agents and MAS gradually improves. Therefore, a 


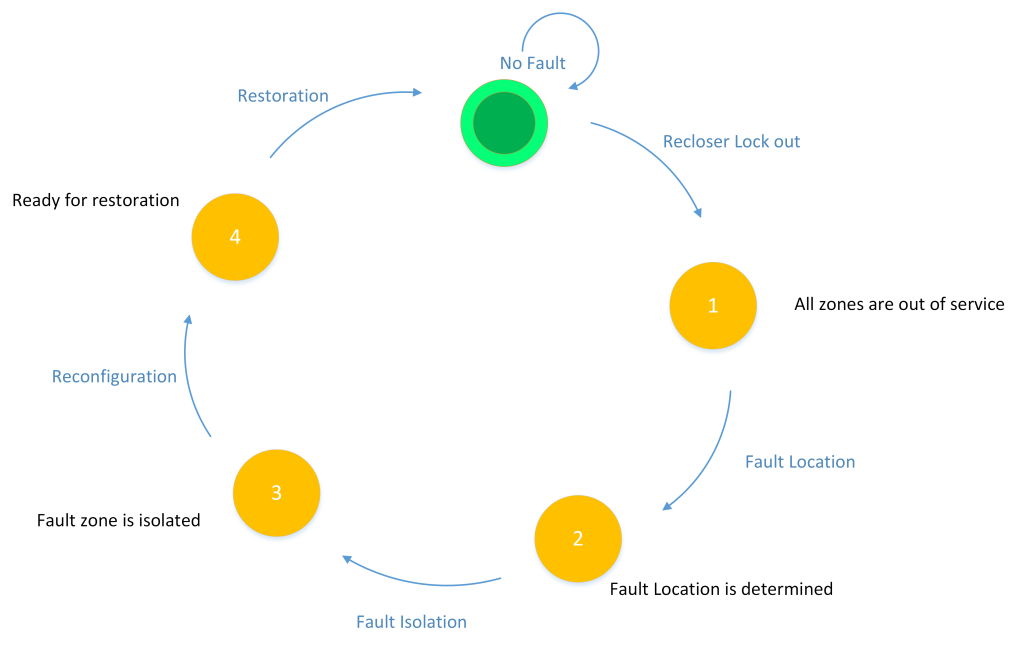

Figure 1.2: Finite State Machine for MAS

learning methodology ( $Q$-Learning) is used to teach agents to use their experiences and prevent them from doing the time consuming optimization process each time a fault happens.

\subsubsection{Dissertation Outline}

The outline of the remaining chapters is given in this chapter.

In Chapter 2, we are giving a literature overview of the most significant research work in the area of FLIR and multi-agents system application in power distribution systems. The possible MAS architectures and their advantage and disadvantages are discussed in Chapter 3 along with the MAS communication requirements in PDS. Chapter 4 introduce the proposed Fault Location and Isolation (FLI) approach along with the FLI formulations. In this chapter also simulation results and relevant discussions are given.

In Chapter 5 the proposed restoration and reconfiguration flowchart are presented and restoration process is illustrated. In Chapter 6 the basic idea of reinforcement learning ( $Q$-Learning) method is explained and the modified $Q$-Learning approach for restoration decision making is described. In Chapter 7 , the simulation models which are used for evaluating the proposed FLIR approach are presented and the performance and feasibility of proposed framework is demonstrated on West Virginia Super Circuit (WVSC) which is composed of 5 distribution feeders. Chapter 8 investigates the communication performance of the proposed FLIR approaches and also compare its performance with some similar recent works. 
If the sum of DG capacity and available power from other feeders is less than the required power for restoration of all loads, load shedding can be done to restore the service to as many loads as possible. Chapter 9 presents a distributed load shedding algorithm that finds the minimum amount of the load to be shed before restoration.

Development of a test system platform, capable of emulating a present day power system and MAS will help in dealing with real-world implementation challenges. Chapter 10 presents the development of a platform comprising of meters, agent components, loads integrated with a power system platform to verify applicability of proposed method in locating and isolating the fault using a real world case study.

Finally, conclusions and future works are detailed in Chapter 11.

\subsection{Publications}

References [9-14] are the works published or submitted from this research. 


\section{Chapter 2}

\section{Literature Review}

\subsection{Multi-Agent Systems}

Multi agent system is one of the popular approaches for decentralized management of power systems and is applied to different areas like fault diagnosis, voltage stability, electricity market pricing, protection coordination, power system reconfiguration and restoration. In most approaches for fault location in the literature; a master agent makes the final decision based on the data received from other agents [15]. In some other works each agent is considered to have its special functionalities e.g. acquiring data, analyzing data, managing situations, etc., and each step of decision making is done by a special agent.

Proposed multi agent system in this work is different in the way that each agent has the decision making ability. The method introduced in this work uses voltage and current rms values and also the impedance calculated by voltage and current to monitor the network and identify the faulty zone and fault type. The advantages of this method are that each agent can work independently in case of loss of connection and it needs less communication capacity because agents just talk to their upper level adjacent agent. There are two main approaches for modeling MAS in power system. MAS and power system model could work together in real time mode or offline. An interface plays a key role in connecting two softwares. Developing interfaces between power simulation models and software agents is a key part in the works described previously. In [16, 17] authors describe the Electric Power and Communication Synchronizing Simulator (EPOCHS); EPOCHS is a simulation system, which links the power simulators with an event-driven 
communication network simulator. Another implementation to integrate software agents with simulation model of electrical system is presented in [18], Common Object Request Broker Architecture (CORBA) is used to communicate MAS with an EPDS model in Virtual Test Bed (VTB) simulation environment.

Softwares used for modeling the MAS and power system can work together in two ways:

\section{Real time:}

In this case power system model and MAS should be able to communicate in real time and the data is accessible to agents without any delay in simulation time. Since mostly previous works $[16,19,20]$ used two different softwares for modeling MAS (JADE, EPOCHS...) and power system (OpenDss, EPDS, ...), there are some problems with interfacing two softwares. In this work both MAS and power distribution system are simulated in MATLAB and there is no need for interfacing two softwares.

\section{Offline:}

Some authors use offline data for power system simulation as inputs for MAS.

In this case the model cannot consider communications in exact time like the real world.

In recent years, much research interest has focused on each aspect of a self-healing PDS i.e. Fault Location, Isolation, Reconfiguration and Restoration (FLIR) and some techniques are developed to solve each problem. In next sections of this dissertation several available FLIR algorithms are discussed. However, a key disadvantage is the fact that most of these approaches are centralized. For example, Nagata et al. proposed a centralized solution for power restoration [21, 22]. Due to complexity and expansion of present-day distribution systems, conventional centralized and regulated control systems tend to be inadequate because of dependency on powerful central computing facilities to handle huge amount of data with high communication capabilities, which can lead to a single point of failure.

To this end, distributed methods such as Multi-Agent Systems (MAS) have received significantly increased attention recently in the community to handle the complex FLIR research and development [21-23]. Decentralized MAS can overcome the disadvantages of centralized approaches because of their decentralized 
data processing, which leads to efficient task distribution and eventually causing faster operation and decision making process. Thus, avoiding a single point of failure, while utilizing peer to peer communication for collaborating to achieve near global goals. Therefore, MAS technology for FLIR seems to be quite promising and the utility industries will benefit from it since there will be no need for exclusive central control.

In [24], Solanki et al. proposed a decentralized MAS to restore the power supply to de-energized loads. Although the MAS framework is decentralized and can avoid the single point of failure, the accurate decision cannot be guaranteed, because agents only have a limited view of the system states. Moreover, it requires a lot of communications which are time consuming. To overcome this shortcoming, references [23, 25] provided solutions. Reference [23] used a hybrid MAS architecture to take the advantage of both centralized and decentralized approaches. In that framework, although agents have a broader view of the power grid, they still use the central agent suggestions for their decision making. Moreover, their proposed MAS architecture requires large number of message communications which hinder the reliability of the MAS. Reference [25] proposed a dynamic team forming algorithm which tries to solve the restoration problem locally and if not possible, enlarges the group size. Dynamic team forming and enlarging the group size could be time consuming processes.

\section{$2.2 \quad$ Fault Location \& Isolation Methods}

Considering the existence of multiple laterals and sub laterals tapped off the main feeder in different locations of PDS, time varying unbalanced load profiles, power injection at different location of system by distributed generation, fault locating in PDS is a challenging task. In [26] the algorithm constantly monitors the network and from the available observations identifies the fault location. In [27], authors used sequence current magnitudes and current direction during a fault were used for fault detection. Previous works on Fault Location and Isolation (FLI) could be categorized in three main categories;

1. Impedance based methods: Impedance based methods; these methods usually calculate the apparent impedance sequences using measurement 
points data and estimate the possible fault locations based on iterative algorithms. Considering the multiple fault locations estimation in these methods and existence of many laterals in PDS is the drawback of impedance based methods [28-30].

2. Wavelet based methods: Wavelet based methods, in which discrete Fourier transform or wavelet transform are used to analyze the fault waveform. It's difficult to guarantee the reliability of these methods because of variety of load characteristics and fault cause in PDS [31].

3. Intelligent methods: Intelligent methods consist of artificial neutral networks (ANN), Expert systems and etc., ANN based methods need to be trained after any change in system and update the network weights, the other drawback with ANN based methods is that in case of complicated networks they became slow and also need to be trained after changes in network $[32,33]$. Expert system methods have a slow response time since they involve knowledge based maintenance and conventional inference mechanism [34].

Below is a brief review of some major existing intelligent methods [35].

\subsubsection{Expert Systems (ES)}

Expert systems were the earliest attempt in Artificial Intelligence (AI) applications to power system fault diagnosis. In rule-based expert systems, knowledge of the power system is represented as rules stored in the database via if-then-else form. After the information of operated relays and tripped CBs are inputted, the fault section is estimated by the inference engine. Although the rule-based ES offers a useful method for fault diagnosis, common drawbacks of ES-based fault diagnosis involve knowledge-based maintenance and slow response time due to conventional knowledge representation and inference mechanism.

\subsubsection{Artificial Neural Networks (ANNs)}

In the application of fault diagnosis, artificial-neural-nertwork (ANN) models can be regarded as an opaque black box that performs a special case of vector mapping. This mapping is opaque as we do not understand how it works. The main 
advantages of ANN for fault diagnosis are its flexibility with noisy data and fast response time. No explicit rules are required to precisely define the power system configuration and protective schemes. However, it needs an additional training process and takes time to derive the required network weights. When power systems become more complicated, the convergence of the training slows down and sometimes falls in a local optimum. In addition, when the changes in power networks occur, neural networks should be trained again in response to each change.

\subsubsection{Petri-Nets $(\mathrm{PN})$}

The major features of the Petri net (PN)-based methods are the abilities of graphical knowledge representation and parallel information processing. A basic PN consists of four basic elements: 1) places, 2) arcs, 3) transitions, and 4) tokens. For a given PN, its topological structure can be represented by an incidence matrix. The reasoning of rule-based systems can be realized through matrix operations. The PNs have to be marked with tokens to be used to perform further analysis. In general, the incidence matrix becomes large in dimension for a complex power system, leading to the difficulty in analysis. In this situation, a matrix reduction technique and an efficient inference algorithm are required to make PN-based methods applicable to power systems.

These approaches are mostly centralized and try to locate the exact fault location which is not the case for this research. Recently, with the development of digital sensors and information communication technologies, new FLI techniques can be applied to PDS. Since agents in MAS continuously exchange information, it makes it possible to determine the faulty zone by local information. In the proposed method, each ZA, according to the rms value of the measured current waveform of itself as well as its neighbors, determine whether there is a fault in its zone or not by monitoring the changes in the current rms values $[12,13]$.

\subsection{Service Restoration}

Once the Fault is isolated, the optimal load restoration decision can be obtained using combinatorial optimization problem solving techniques [11, 36, 37], heuristic search techniques [38], network flow [39] or graph theory techniques [40]. Dynamic 
programming is also one of the commonly used techniques for this kind of problems [37]. In a paper by authors [11], the restoration problem was formulated as a Knapsack problem. That work uses the similar approach where the problem is modeled as mixed integer linear optimization approach for which the optimal solution can be easily obtained.

Not surprisingly, much research attention has focused on automated discovery of optimal power system configurations for shipboard power systems (SPS). One class of algorithms for shipboard power systems reconfiguration are designed to take actions by means of distributed software agents [24, 41]. Requiring no centralized processing, these approaches rely entirely on local computations to reconfigure the SPS. Unfortunately, multi-agent systems that rely on local communications might not yield the best possible configuration unless optimal reconfiguration is cast as a convex optimization problem without local minima. As a trade-off of optimality over speed, many other approaches are designed to produce the optimal configuration. Both stochastic and deterministic approaches that have been investigated for this purpose, are discussed with more detail in the following.

\subsubsection{Heuristic Techniques \& Knowledge Based Systems}

The heuristic approach is intuitively a rule of thumb, to limit the search space. The expert's knowledge and experience are translated into programming logic to solve the problem. The drawbacks of heuristic methods are:

1. optimal solution is not guaranteed;

2. they have great difficulties in maintaining the software, since the size of the software is large and the algorithm is complex

Expert system is basically a knowledge-based technique. It involves the representation of expert knowledge as rules and an inference engine to infer from these rules. The rules are written as IF-THEN statements. Expert system approaches can be considered as successful approaches to solve the restoration problem; however, maintenance of large-scale expert systems has turned out to be costly. In addition, expert system rules are system specific, and they change with the system. Heuristic techniques and knowledge based systems utilize the operator's knowledge and experience to narrow down the search space for the combinatorial restoration 
problem. Thus, the solution is achieved in a shorter time. In [42], Liu et al. reported an expert system algorithm for restoration and loss reduction of distribution systems. The authors constructed a knowledge base which contains rules that implement a solution approach that system operators can use in order to restore as many loads as possible. An early work on heuristic search approach to distribution system restoration was introduced by Morelato and Monticelli [43]. The proposed approach used a knowledge guided search strategy to reach the solution. Artificial neural network (ANN), ant immune system-ant colony optimization (AIS-ACO), Genetic Algorithm (GA), among others are also used extensively for restoration problems.

However, most of the restoration techniques surveyed above solve the reconfiguration problem from a centralized point of view, which in reality often requires a low-latency communication system transferring a potentially large amount of data between field devices and the control center. In addition, the control center requires expensive computing capability. As a result, centralized techniques are subject to a single point of failure risk and large capital costs.

\subsubsection{Mathematical Programming}

The mathematical programming approach determines the target configuration for restoration often by formulating the problem as a mixed integer non-linear problem (MINLP). Each branch represented by a binary variable (0: opened \& 1: closed). Other constraints such as supply and demand balance are formulated in terms of continuous variables. Then, any available MINLP technique can be applied to solve the restoration problem. Nagata et al. [22] proposed a two-stage algorithm which decomposes the restoration problem into two subproblems (the maximization of available power to the de-energized area, and the minimization of the amount of unserved energy). The algorithm is limited to dc models which do not take into account the reactive power as well as voltage variations. In [44, 45], the authors presented the network reconfiguration for service restoration in shipboard power distribution systems. They solved optimal restoration of distribution systems using a dynamic programming approach. During the solution process, many states close to each other are grouped. Then, only the best states are selected to reduce the problem size, so that solution speedup is achieved. While 
mathematical programming might guarantee a global optimal solution, its drawback is the computational intensity, so it might not be practical for centralized large distribution systems.

Multi-agent systems (MAS) are one of the most interesting new fields of computer science and distributed artificial intelligence. MAS are composed of multiple interacting computing elements, known as agents. These agents are located in some environment and react to the changes in this environment and are capable of acting (taking decisions) in order to achieve their goals. Multi-agent system can be considered as the plate form of distributed processing, parallel operation, and autonomous solving. Further, it can be much faster in solving discrete and nonlinear problems. Therefore, MAS as a distributed restoration algorithm represents an interesting candidate for restoration and control of the distribution system to realize the self-healing operation.

\subsection{MAS Communication}

A communication system is an essential part of the MAS and plays a crucial role in construction and operation of agents. The communication system is responsible for data exchange among the agents and there are specific communication requirements for different application in power system such as wide area monitoring system (WAMS), protection (WAPS) and control system (WACS) [46]. Each of these application require different response time, data exchange volume and etc., and the communication system should provide the special required bandwidth, latency, reliability for each application. In this dissertation we are going to explore the challenges for communication media in MAS and investigate the effect of communication media on different MAS architectures for fault detection and isolation in power distribution systems. A fault in the distribution network often interrupts the electric power to all the consumers along the feeder. The reliability of the supply can be improved in a fast and cost-efficient manner if we could do the fault location, isolation, reconfiguration and restoration operations faster. One of the most common protection methods used for radial distribution systems is having a recloser at the substation which will isolate the feeder in case of short circuit faults. Whenever a fault occurs the recloser opens and closes three times to check whether the fault still exists in the feeder or not. If the fault does not clear after these attempts, it locks out and disconnects the power of the whole feeder. This 
problem may be solved by identifying and isolating of fault before the recloser has been opened for the lock out status. Agents in power systems can heal (isolate) the fault through communicating with each other. Communication media for this application can be based on wired communication technologies such as fiber optics, power line communication (PLC) systems, copper wire line or wireless technologies such as cellular networks (i.e. GSM/GPRS/WiMax/WLAN). This dissertation investigates the latency and reliability of centralized and decentralized MAS approaches in fault detection and isolation applications of power distribution systems for wired and wireless communication technology scenarios. This study requires an integration of power system and communication network models to simulate the performance of different control strategies considering communication between agents. Continuous time simulation of power system and discrete event simulation of communication network have to be integrated. Although co-simulation of power system and communication network behind it is a relatively new topic in power system applications, there are some works done in this area [47]. EPOCHS is an approach for building a power system modeling tool considering the underlying communication network. In this method, simulators for power system and communication run separately and halt at fixed synchronization points. A similar work is also reported in [48]. In [49], Matlab Simulink and OPNET are used to study the communication system on the reliability of WAMS applications. In this work we used S-functions in Simulink software to model the communication between agents and there is no need for synchronizing the communication and power system softwares since they are both using the Simulink engine to run. The S-function callback methods used for modeling communication system, perform tasks required at each simulation stage. During simulation of a model, at each simulation stage the Simulink engine calls the appropriate methods for each S-Function block in the model.

\subsection{Learning Algorithms}

Although the agents in MAS can be programmed with behaviors designed in advance, it is also necessary that they learn new behaviors online, such that the performance of the agents and MAS gradually improves.

In this dissertation, the novel concept and detailed system structure of self-healing power distribution grids with learning capability is proposed. MAS use an integer 
non-linear optimization method to choose the configuration that maximizes the weighted current delivered to the loads and learns from its behavior for future decision makings; preventing the time consuming optimization process each time a similar failure occurs. Q-learning which is a popular reinforcement strategy learning approach is used widely to help agents learn from their experiences[50]. $Q$-Learning algorithm which is a reinforcement learning algorithm was proposed by Watkins to deal with Markov Decision Problems (MDP) [50]. It has been extensively used to solve the strategy control and learning problems of autonomous agents. Authors in [44] used $Q$-Learning for dynamic reconfiguration of shipboard power systems. In [23] $Q$-Learning is used for power grid systems restoration. The $Q$-Learning technique introduced in this dissertation is modified for power distribution systems and uses $Q$-Matrices as the repositories for all the information learned through previous restoration experiences.

It is an online learning approach, which not only let agents to tune themselves with time but also re-adapt to the non-stationary environment. This makes Qlearning less susceptible to system drift due to equipment aging, replacements and etc. In [39, 44] and [36] Q-learning techniques have been used for shipboard and distribution power system reconfiguration and restoration. The main noteworthy contribution of this research is the modified Q-Learning approach which specifically is designed for self-healing power distribution systems. And also the methodology that is used to teach agents to use their experiences and update their knowledge as the system is running.

A Q-learning agent learns by interaction with dynamic power system environment and does not need a teacher. At each time step, agents perceive the complete state of the system and take an action. Agents learn by evaluating the quality of the transition in the system state due to the taken actions. With learning and when agents are holding much information, they become confident about their decisions and can do the restoration faster and without doing the optimization process.

\subsection{Load Shedding}

Power outages in distribution systems can create power mismatch between generation and loads. Power balance in an electricity grid must be maintained on a second by second basis since electricity cannot be stored easily in a cost effective 
manner. When a fault occurs, firstly the fault should be located to be able to isolate the faulted zone. Secondly, the service should be restored to the loads which are in the fault-free areas.

The ability to control the load is important when running an electric power network. Thus Load Shedding (LS) and Demand Response Management (DSM) programs are becoming increasingly widespread among electrical users. DSM is specifically devoted to peak demand shaving and to encourage efficient use of energy. LS is a methodology used to prevent from overloading the power system to keep the frequency and voltage profiles in the standard boundaries and acts in a preventive way [51]. In order to do the load shedding program, it is necessary to have priority classification for the PDS customers.

The diffusion of Distributed Generation (DG) can be a further opportunity to improve the management of the electric system. It is widely expected that DG will have a significant contribution in the PDS, where small generating sources located close to the loads are dispersed in distribution networks. It means many customers can be supplied by the distributed resources. The adoption of DG's can considerably increase the reliability of the PDS in case of outages.

The objective of this work is to develop a distributed Multi Agent System (MAS) to restore the power to high priority loads after the outages happen. After fault location and isolation using the available techniques $[12,13]$, MAS tries to find the optimal reconfiguration to re-energize the fault-free zones. In general there are three ways to restore the service for fault free zones,

1. Direct feeder restoration: the zones upstream the fault zone can be restored by reclosing the recloser at the top of each feeder.

2. Load transfer restoration: If other feeders have enough extra power to provide for out-of-service areas, and there are tie switches that can transfer the power.

3. Load-shedding restoration: load shedding restoration is when there is not enough available power to restore all the out-of-service zones. If there are Distributed Generation (DG) sources in the out of service zones, they can be used to restore some loads. If the sum of DG capacity and available power from other feeders is less than the zone's power demand, load shedding can be done to restore the service to as many loads as possible. 
It is possible to estimate the load that must be shed before restoration switching in order to keep the power balance in the system. The traditional load shedding strategy is to shed a fixed amount of load when the frequency is decreasing. Fastacting load shedding is presented in [52]. Supervisory Control and Data Acquisition (SCADA) based load shedding strategy has been presented in [53]. Another load shedding strategy with online measurement of loads' frequency is discussed in $[54,55]$.

It is difficult to determine the amount of the load to be shed when the system loads change dynamically and has significant penetration of generations that are stochastic in nature, such as wind turbines, solar cells, etc. In this dissertation, authors presented a distributed MAS-based system that finds the near-optimal configuration for restoration using the available local data. A new fuzzy-based load prediction methodology has been used in this work that allows agents to predict their future load profile and update their knowledge with the online monitoring data. More detail on the proposed load shedding algorithm is presented in Chapter.9.

\subsection{Available FLIR Systems}

Until the 1990S, technological breakthroughs in the automation of electric distribution systems were slow to materialize. Early SCADA (supervisory control and data acquisition) systems promised to improve reliability. But many of the early systems were little more than an extension of SCADA beyond the substation fence. With their relatively low computational and communication capabilities, these early systems required a high degree of human intervention and offered little in the way of true automation. As a result, expectations were not always met, leading to the termination or scaling back of many projects. Nevertheless, many lessons were learned and technology has advanced, especially through the 1990s and to the present. More advanced automation applications have been developed that are far more practical and manageable for utilities to implement. Some tools for feeder automation have been available for many years. Below we presented the S\&C Electric Company product (IntelliTEAM - SG) which is a patented and commercialized product and compare it to the proposed FLIR system presented in this dissertation $[1,56]$. 


\subsubsection{Intelliteam SG $[1]$}

In 2003, the IntelliTEAM Automatic Restoration System was introduced by S\&C Electric Company. IntelliTEAM II is a patented automatic power restoration system that uses distributed intelligence and peer-to-peer communication to switch and isolate the faulted line section and restore power to unfaulted line sections.

IntelliTEAM utilizes ties to multiple circuits, enabling a wide variety of circuit configurations to maximize the restoration potential. Distributed intelligence prevents line overloads. IntelliTEAM can automate parts of a circuit in need of attention or an entire distribution system consisting of open-loop feeders with multiple tie points to other feeders. It can be used both tactically and strategically, based on the reliability problems the utility needs to solve .

\subsubsection{IntelliTEAM Theory of Operation}

IntelliTEAM dynamically tracks system conditions on overhead and underground distribution systems and provides fully automatic fault isolation and service restoration. "Team" and "coach" metaphors help to describe IntelliTEAM operation. Each team is defined as a line segment bounded by two to eight automated switches, each of which can represent a different power source. Each team has a software coach. If an outage or line fault occurs, local control logic determines if a switch should open. The coach of each affected team then uses real-time data, including voltage and current data gathered prior to the system event to develop a restoration strategy for unfaulted sections. The coaches of adjacent teams then work together through shared controls to implement strategies that will maximize restoration of the circuit within the prioritization rules defined by the user.

Breakers and reclosers can also be incorporated as team members with the addition of a module that interfaces to the electronic relay or control that operates the breaker or recloser.

Fig. 2.1 illustrates the team concept. The coach distributes data and coordinates the operation of automated switches, reclosers, and breakers. Coach travels to each team member within a prescribed time period. Coaches are responsible for restoration decision makings and they move to each switch that has the capability for restoration and ask the neighboring team coach to move to the same switch to 
make restoration decisions. When a coach moves to a team member to investigate the restoration possibility, it searches for the neighboring coach from the adjacent teams.

In the event of a loss of communication, service restoration may be inhibited, but an automated switch, recloser, or breaker can still isolate a fault based on local fault detection and isolation software logic.

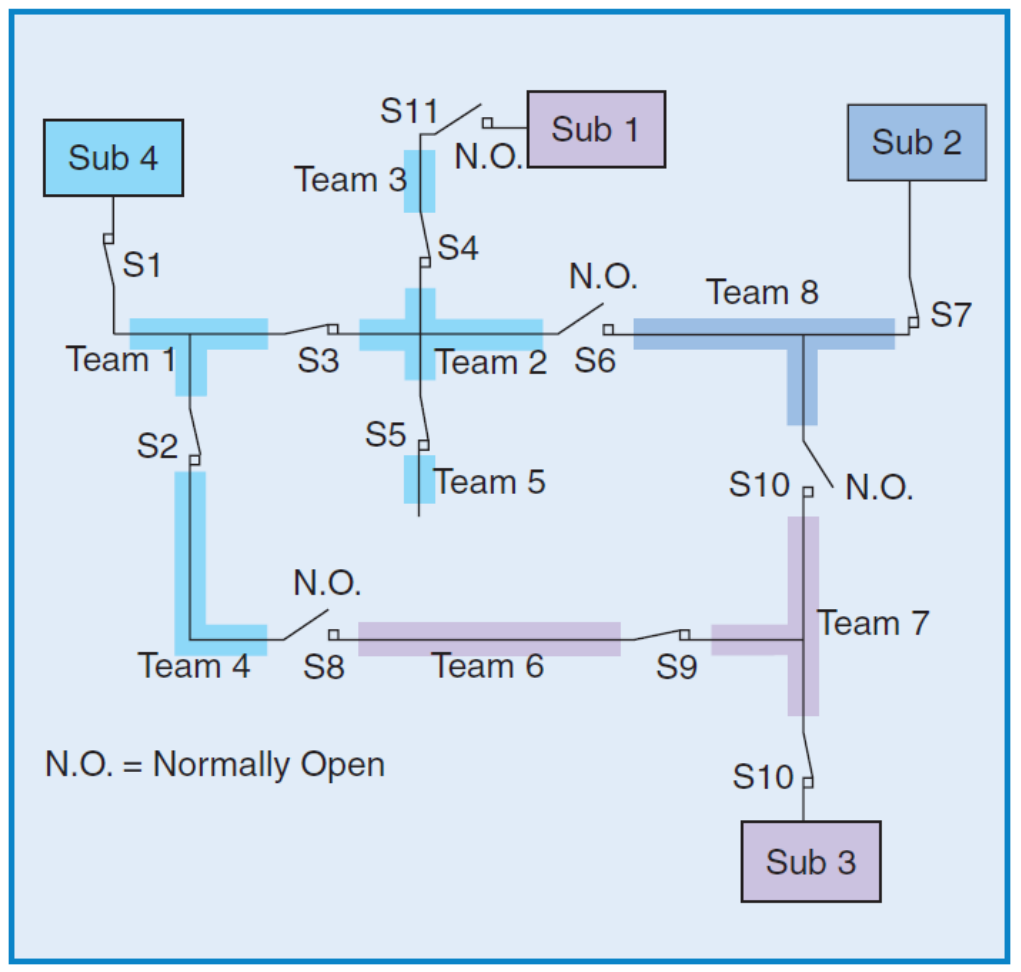

Figure 2.1: An EXAmple of InTElliTEAM USED to AUTOMATE FOUR OPEN-LOOP DiSTRIBUTION CIRCUITS

At the beginning of a fault or outage event, any team member detecting the event will communicate to all the other team members and the coach, reporting the time, type of event, report identification, and so on. The coach also monitors the status of each device, ensuring that all are in the right open or closed position to allow progress to the next restoration step.

In order to close a switch, the coaches of adjacent teams must grant permission based on circuit conditions, location of fault, and an evaluation of loading conditions. When all appropriate conditions are met, the switch can be closed to restore service to a feeder segment. This process is repeated for each segment in turn. As switches close, the IntelliTEAM system updates available capacity on the feeders used to restore service, ensuring maximum service restoration even at maximum peak load. 
Following a reconfiguration event, and subsequent repair and restoration of the faulted line section, team members can return to their normal state. Once the "return to normal" is initiated, the coaches begin the process of returning each team to its normal configuration. The return-to-normal process begins at the team closest to the normal source and works outward, using either an open or closed transition approach as defined by the user.

To support the distributed control architecture of IntelliTEAM, a peer-to-peer wide-area communication network is essential. There are only a few commercially available wide-area communication systems that readily support such a network. Typically, communication for the IntelliTEAM system is provided by $900-\mathrm{MHz}$ UtiliNet spread-spectrum radios. The UtiliNet radio system transports data using a connectionless mesh architecture. This architecture allows each radio in the network to act as both a repeater radio and a data transceiver for an automated switch. The radio network is highly scalable and obviates the need to invest in the tall master radio towers prevalent in point-to multi point radio networks.

Fiber-optic networks have also been utilized for high speed peer-to-peer communication, typically in underground applications where fiber is installed along with the underground cable infrastructure.

\subsubsection{FLIR \& IntelliTEAM}

In Intelliteam the local control logic determines if a switch should open after a loss of voltage, and/or fault current event. A team member would open to isolate the fault after sensing the fault currents and two times loss of voltage (because of recloser operations). Also, if a switch does not sense the fault current it opens after two times lost of voltage. The fault location can be determined based on the location of switches that sensed the fault current. This FLI method has the following disadvantages:

- It does not support the DG penetration: In case there are DG's in the power system and two way power flow exists, proposed FLI system might not be able to locate the fault.

- It requires threshold settings for fault currents which is challenging and should be modified after any change in the system configuration. 
For restoration, the coach of each affected team uses the real-time data, plus voltage and current data gathered prior to the system event, to develop a restoration strategy for un-faulted sections. The coaches work together to implement strategies that will maximize restoration within the prioritization rules defined by the user. A coach moves from the switch to switch based on the predefined source priorities and checks the restoration possibility through that switch and by negotiating with the neighboring coaches. 


\section{Chapter 3}

\section{Multi Agent Systems}

\subsection{MAS Concept}

In order to explore the potential benefits of MAS to power engineering, the basic concepts and approaches associated with multi-agent systems need to be understood. This leads us to a basic but essential, and unfortunately difficult, question: what is an agent?

\subsubsection{Agent Definition}

The computer science community has produced myriad definitions for what is an agent. While all the definitions share a basic set of concepts: the notion of an agent, its environment, and the property of autonomy [57]. An agent is merely "a software (or hardware) entity that is situated in some environment and is able to autonomously react to changes in that environment." The environment is simply everything external to the agent. In order to be situated in an environment, at least part of the environment must be observable to, or alterable by, the agent. The environment may be physical (e.g., the power system), and therefore observable through sensors, or it may be the computing environment (e.g., data sources, computing resources, and other agents), observable through system calls, program invocation, and messaging. An agent may alter the environment by taking some action: either physically (such as closing a normally-open point to reconfigure a network), or otherwise (e.g., storing diagnostic information in a database for others to access). 


\subsubsection{Intelligent Agent}

An agent which displays flexible autonomy, i.e., an intelligent agent, has the following three characteristics.

- Reactivity: an intelligent agent is able to react to changes in its environment in a timely fashion, and takes some action based on those changes and the function it is designed to achieve.

- Pro-activeness: intelligent agents exhibit goal-directed behavior. Goaldirected behavior connotes that an agent will dynamically change its behavior in order to achieve its goals. For example, if an agent loses communication with another agent whose services it requires to fulfill its goals, it will search for another agent that provides the same services. This pro-activeness is described as an agent's ability to "take the initiative."

- Social ability: intelligent agents are able to interact with other intelligent agents. Social ability connotes more than the simple passing of data between different software and hardware entities, something many traditional systems do. It connotes the ability to negotiate and interact in a cooperative manner. That ability is normally underpinned by an Agent Communication Language (ACL), which allows agents to converse rather than simply pass data.

While an agent, displays the characteristic of reactivity, in order to be classed as an intelligent agent, an agent must also have some form of pro-activeness and some form of social ability. It is the goal-directed behavior of individual agents and the ability to flexibly communicate and interact that sets intelligent agents apart.

\subsubsection{Definition of a Multi-Agent System}

A multi-agent system is simply a system comprising two or more agents or intelligent agents. It is important to recognize that there is no overall system goal, simply the local goals of each separate agent. The system designer's intentions for the system can only be realized by including multiple intelligent agents, with local goals corresponding to subparts of that intention. Depending on the definition of agency adhered to, agents in a multi-agent system may or may not have the ability to communicate directly with each other. However, intelligent agents must have 
social ability and therefore must be capable of communication with each other. For the sake of this work, the authors have focused on MAS where this communication is supported. This clearly differentiates the type of MAS discussed in this work from other types of systems.

\subsection{MAS Architecture}

In order to manage and control the power distribution systems more efficiently, multi agent systems have been employed recently to solve the challenges in current old fashioned power networks. MAS are used for fault diagnostics, system monitoring, reconfiguration and restoration, protection and etc. In general there are two kinds of MAS structures which are centralized and decentralized strategies. In the following more details about both centralized and decentralize approaches and their application in self-healing power systems is presented. It is important to have a common Language for Agent Communication, without which, the coordination and negotiation will hardly be successful. There are some standards for agents' communication languages (ACL) for MAS, among them Knowledge Query and Manipulation Language (KQML) and Foundation for Intelligent Physical Agents (FIPA) are most notable [58]. In these standards the message types have been standardized and the message intent specified. A FIPA ACL message will contain one or more message parameters, according to the requirements of the agent application. In some MAS architecture, agents have different functionalities and responsibilities such as training agents, fault locating agents and coordinating agents and the tasks will be divided among the agents to process [25]. But in this work, agents have all the functionalities and are considered to be autonomous. In general there are two kind of control strategies in controlling agents in MAS, which are centralize and decentralize coordination strategies. Figs. 3.1, 3.2 and 3.3 show these architectures. In the following paragraphs each of them is described with more detail.

\subsubsection{Centralized}

Centralized approaches are mostly conventional and tend to be inadequate for future power systems because of deficiencies in robustness, openness, and flexibility 


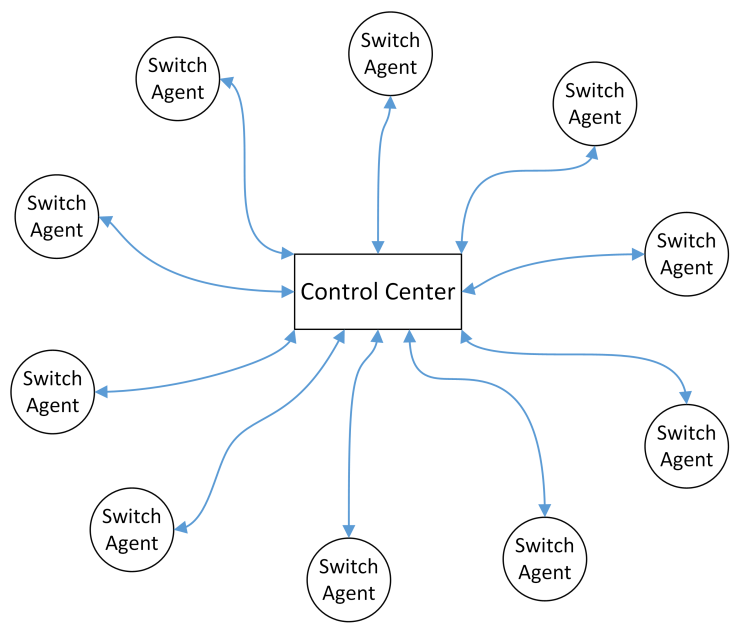

(A) Centralized

Figure 3.1: Centralized Architecture

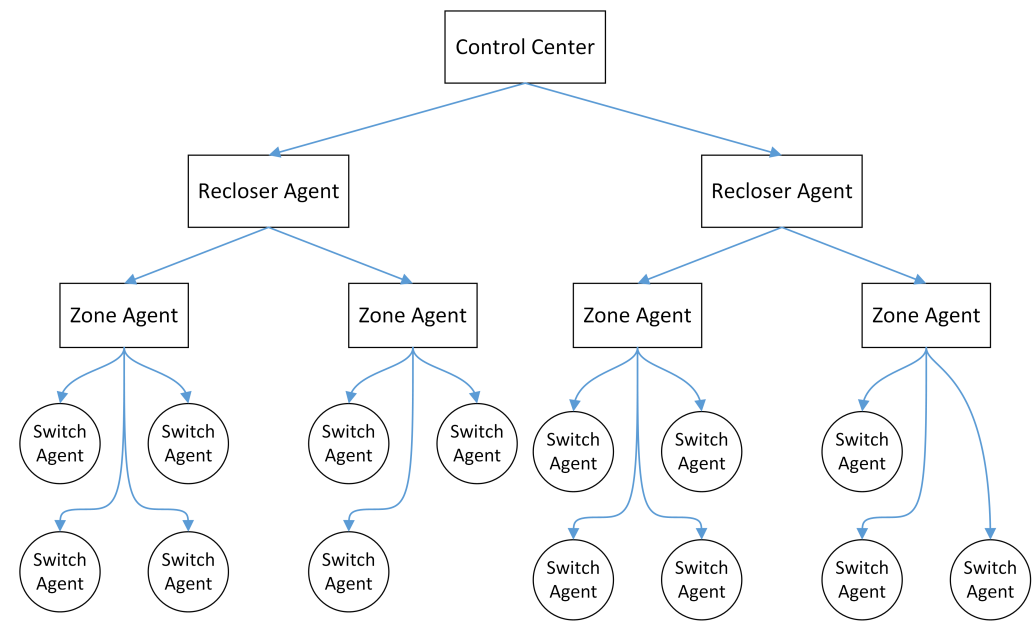

(B) Hierarchical

Figure 3.2: Hierarchical Architecture

and single point of fault. In centralized approaches a coordinator agent is responsible for managing a group of sub-agents. Fig. 3.1 shows the information flow in centralized approaches. In this architecture all the agents send their data to a control center and wait for the commands. Control center runs the fault location and isolation algorithms and make the decisions. Commands will be sent back to corresponding agents through the communication channel. 


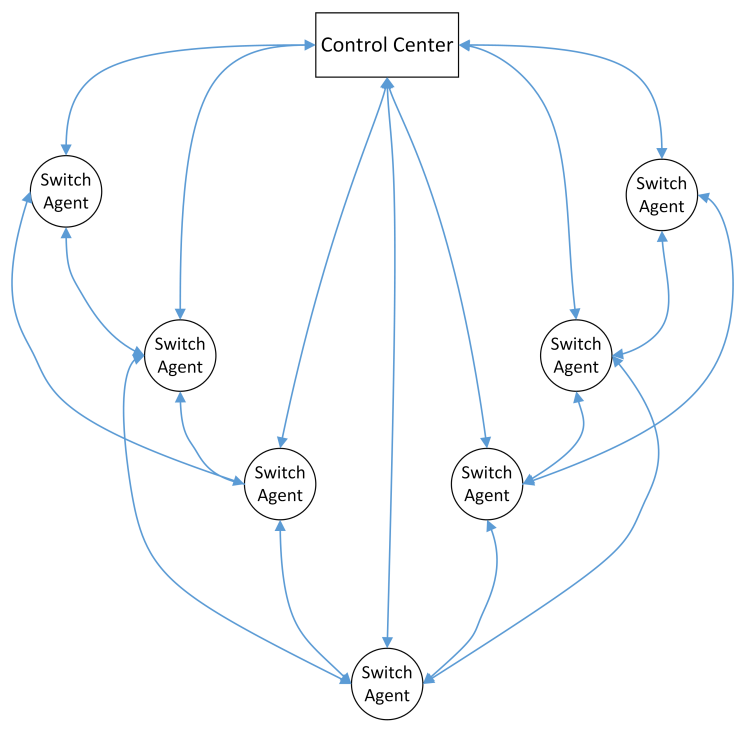

(C) Distributed (Hybrid)

Figure 3.3: Distributed And Hybrid Architectures

\subsubsection{Decentralized}

With the introduction of distributed power generation, load control, market operations, increasing complexity in the distribution network, and an increased number of interconnections, the operation of a modern power system is extremely complex. Multi-agent systems provide a technology for flexibly controlling the modern power system. The current approach of using a central SCADA system and several smaller distributed SCADA systems is no longer sufficient for certain control operations. An approach that provides intelligent, fast, and adaptable local control and decision making is required.

Taking the example of active distribution networks, management and control of complex networks present a number of challenges, not least in the scalability and flexibility of solutions. A number of researchers are considering agent-based approaches as an alternative to centralized power system management and control. By distributing management and control functionality using intelligent agents, decision-making regarding network restoration, reconfiguration, the dispatch of generation, and the management of loads can be locally managed. Local decisionmaking would require agents capable of a range of actions, such as monitoring local conditions, controlling switches and other plant, and coordinating with other regions of the network. 
Although decentralized approaches are more robust and flexible rather than centralized ones but the disadvantage with the decentralized strategies is not reaching the global optimal solutions in all scenarios since the agent's communications are just limited to neighbors. In this approach all agents are in the same level of functionality and communicate with the neighbors to a specified neighborhood. In this architecture agents try to make the optimal decision based on the local condition and in the case of malfunction of any agent, other agents can keep working. Agent's link to control center is just considered as a supervisory monitoring. If the control center also plays a role in decision making, this architecture could be considered as hybrid architecture too.

\subsubsection{Hierarchical}

In this strategy agents are working in different levels of decision makings. Agent's levels from high to low are control center agent, recloser agent, zone agent and switch agent. Each zone agent is in charge of a group of switch agents. Low level agents don't have much authority and just can communicate with higher level agents. Higher level agents have a better overlook over the network condition and can use their upstream agent's comments in their decision making process. The information flow in this architecture is bottom-up and the comments and commands are coming from higher level agents. In this architecture we considered zone agents as decision makers which ask the recloser agent for the permission before sending commands to switch agents. Disadvantage with this architecture is that higher level agent's malfunction can create critical situations for all the sub-agents.

\subsubsection{Hybrid}

Hybrid MAS structures can take the advantages of both centralized and decentralized approaches and reach the optimal restoration plan. In this architecture, MAS works in a decentralized manner but agents will have access to information further than the immediate neighbors in the case of availability. Therefore, they can make better decisions and since they are not just relying on centralized data, even failures in accessing the centralized data do not affect the MAS much. Proposed MAS is composed of hierarchical level of agents which have different responsibilities. 


\subsection{Proposed Architecture}

We first describe the West Virginia Super Circuit (WVSC) in Fig.3.4, that falls into the scope of this work and then MAS architecture, agent types and self-healing process flowchart are presented.

WVSC is divided into different zones connected through 16 controllable switches, shown with circles in Fig. 3.4. A circle illustrate the status of a switch \{black circle(O): closed; white circle $(\bigcirc)$ :open $\}$. Feeder agents are located at the recloser location. The West Run Substation has five feeders, two of which (WR-3 and WR4) are monitored for faults. The substation feeders are equipped with reclosers. When there is a fault in the system, the recloser will go through its trip and close operations as configured.

The entire number of agents in a power distribution system substation corresponds to the overall number of substations reclosers and switches.

The switchs along the feeder are for data acquisition and their role is restricted to record voltage and current waveforms (plus fault location and isolation). Agents are computers with high protection standards and powered by uninterruptible power supplies (UPS) that are installed at some switch locations. MAS is accommodated in these computers.

To perform the FLIR for a self-healing PDS, the hierarchical distributed MAS framework is employed. As shown in Fig. 3.5, there are three type of agents in the proposed MAS, i.e., $Z A, F A$ and $S A$. These agents are intelligent units that have problem solving capabilities and can communicate, resolve, coordinate and debate with other agents and make decisions. Zone agents are the lowest level agents which are in contact with their neighbors. Each feeder agent is in charge of a number of zone agents and communicates with the other feeder agents. Substation agents just have a communication link to their feeder agents and also neighbor SAs. 


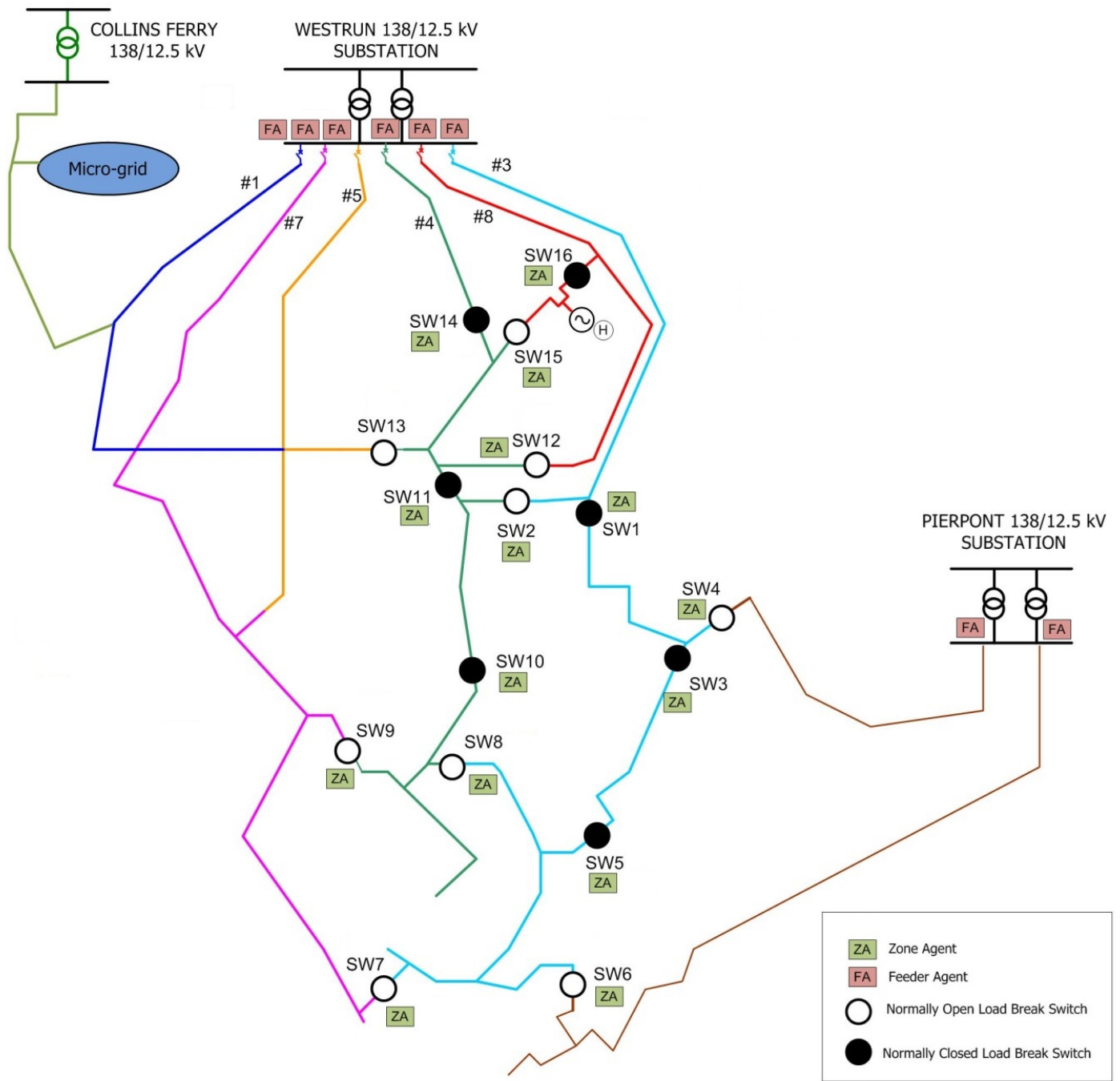

Figure 3.4: West Virginia Super Circuit

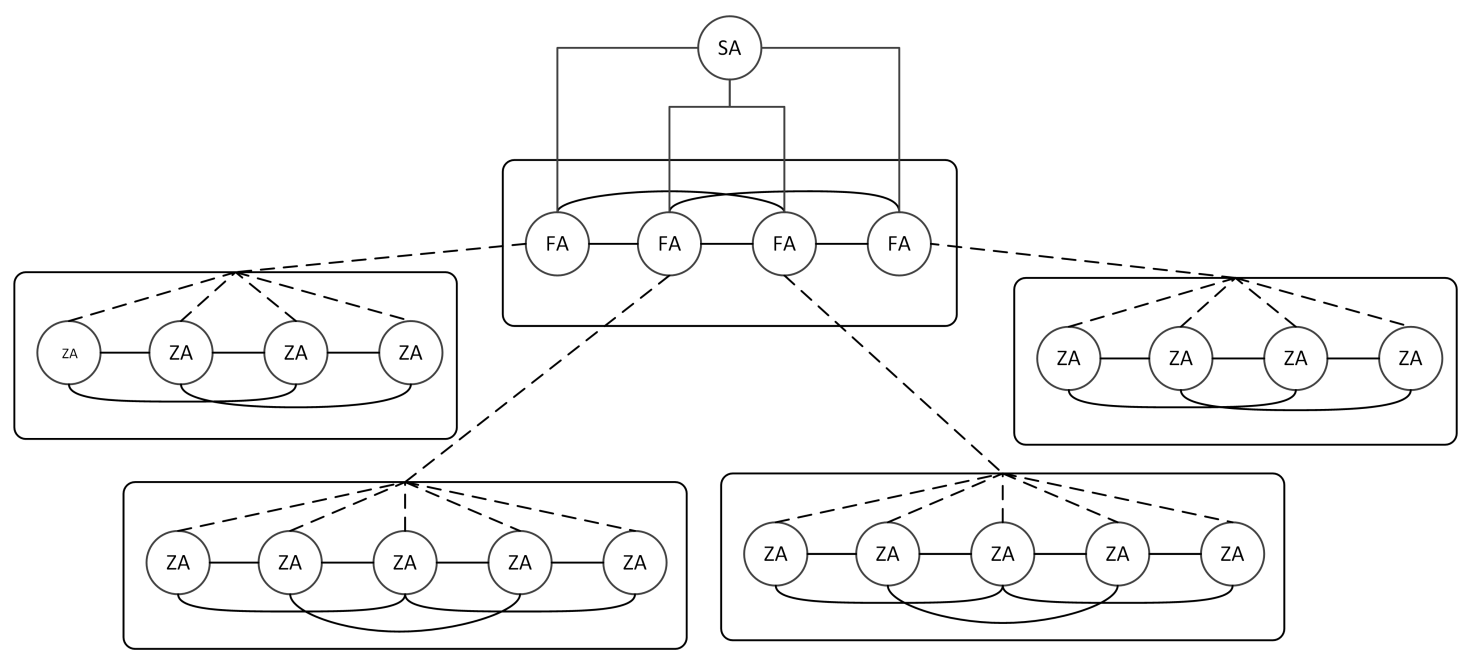

Figure 3.5: MAS Structure 


\subsubsection{Agents}

\subsubsection{Zone Agent (ZA)}

Distribution feeders are divided into sections by the installed switches along the feeder. The physical switch at the top of each section represents the corresponding zone agent. ZAs can directly communicate with their neighbor ZAs and also corresponding FA.

A ZA monitors the status of the corresponding zone voltage, current waveforms and calculates its current usage. ZA contains a load profile estimation function that provides a mechanism for them to predict the future zone load which is required for the FLIR algorithms.

\subsubsection{Feeder Agent (FA)}

A FA is located at the top of each feeder in the recloser location. When FA is provided with the fault location by ZAs, it starts negotiation with potential alternate sources and decides whether to solve the mathematical optimization problem or use the learning model solution based on the available knowledge about the system's state.

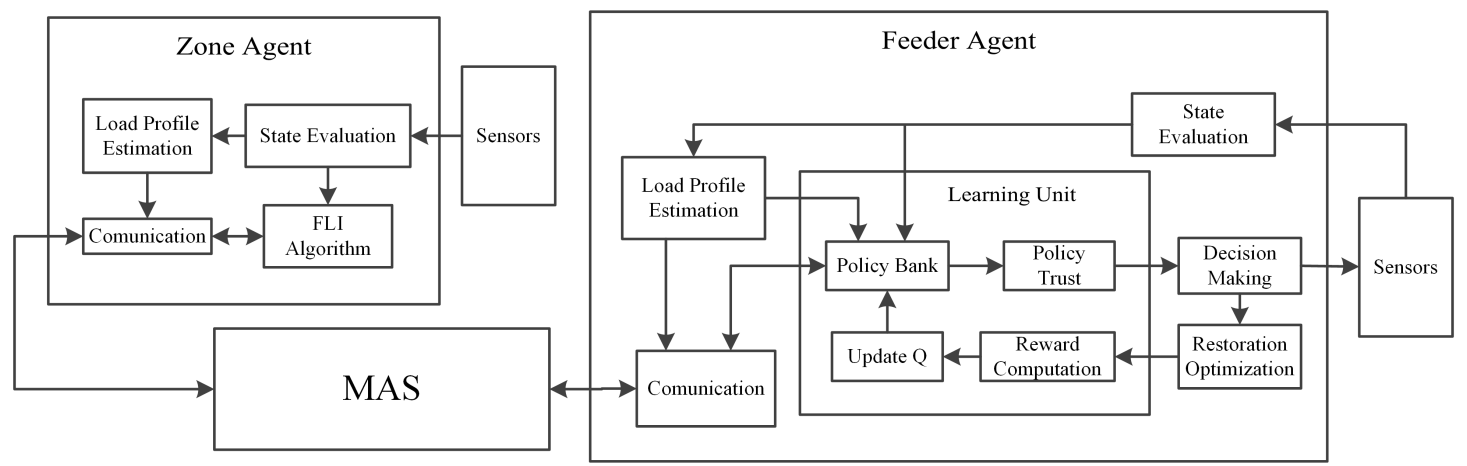

Figure 3.6: ZA And FA Structures

In Fig. 3.6 the procedure of a FA operation is illustrated. Firstly, the FA monitors the system state through measured data by the meters installed at the recloser location. FA is responsible for restoration of its zones in case a fault occurs in any of the downstream zones and contains functions for learning, restoration optimization, load prediction and communications. Each function is discussed in detail in the rest of dissertation. 


\subsubsection{Substation Agent (SA)}

The role of the SA is to negotiate with other SAs which have tie switches to faulty zones. They provide the required data from other substations for their faulted FAs.

\subsection{MAS Communications}

Different communication technologies supported by two main communication media, i.e., wired and wireless, can be used for data transmission between agents. Each of the wired and wireless technologies has their own advantages. Wired technologies are more expensive but don't have interference. Wireless communications have the ease of connection to difficult areas and are cheaper. In the following sections the time delay and reliability of wired and wireless communication media are discussed.

\subsubsection{Wired}

Wired technologies such as fiber optics, Power Line Communication (PLC) systems, copper-wire line are used in existing Wide Area Networks (WANs). Among the wired technologies, Power Line Communication (PLC) could be a promising communication technology for MAS communications [59]. However because of noisy environment and low capacity of narrowband PLC, its viability is questionable.

\subsubsection{Wireless}

Wireless communication technologies like ZigBee, 6LowPAN, Z-wave, WiMAX, $4 \mathrm{G}$ and $3 \mathrm{G}$ cellular networks and etc. are the choices which can provide the data flow among the agents. Long range communication is usually achieved through technologies like WiMAX and cellular networks, whereas short range applications (in-home) use technologies like ZigBee [59]. Although these technologies have different characteristics in this work we just assume an average range of delay and reliability values for our simulation. In this work we considered the Routing and 
media access latency of 5 - 500 milliseconds, the serial delay is assumed to be less than 2.5 milliseconds and the inter packet delay 1 millisecond.

\subsubsection{Communication Languages}

Mechanisms for the communication between agents underpin their social abilities. As agent technology has matured, a number of different methods for interagent communication have been developed. One of the first Agent Communication Languages (ACL) to be used by different researchers across different fields was the Knowledge Query and Manipulation Language (KQML), which emerged in the early 1990s through the U.S. government's DARPA knowledge-sharing program. In recent years, KQML has been superseded by FIPA-ACL [58]. FIPA-ACL message contains 13 fields (see Table 3.7). The first and only mandatory field in the message is the performative field that defines the type of communicative act or speech act. By classifying the message using a performative, FIPA-ACL ensures that recipients will understand the meaning of a message in the same way as the sender, removing any ambiguity about the message's content.

Table 3.1: FiPA-ACL Message Structure

\begin{tabular}{|c|c|}
\hline Message Field & Description \\
\hline performative & type of communication act \\
\hline sender & participant in communication \\
\hline receiver & participant in communication \\
\hline reply-to & participant in communication \\
\hline content & content of message \\
\hline language & content language \\
\hline encoding & encoding of content \\
\hline ontology & ontology used \\
\hline protocol & protocol for conversation \\
\hline conversation-id & ID for conversation control \\
\hline reply-with & conversation control parameter \\
\hline in-reply-to & conversation control parameter \\
\hline reply-by & conversation control parameter \\
\hline
\end{tabular}

Fig. 3.7 illustrates the flow of messages specified by FIPA for a query-ref interaction. For example, agent A may be interested in the details of distributed generators currently connected to a local MV network. If agent D is responsible 
for the management of that network, agent A could ask agent D for details of all the cases it knows of where local generators are currently connected, by using the query-ref communicative act.

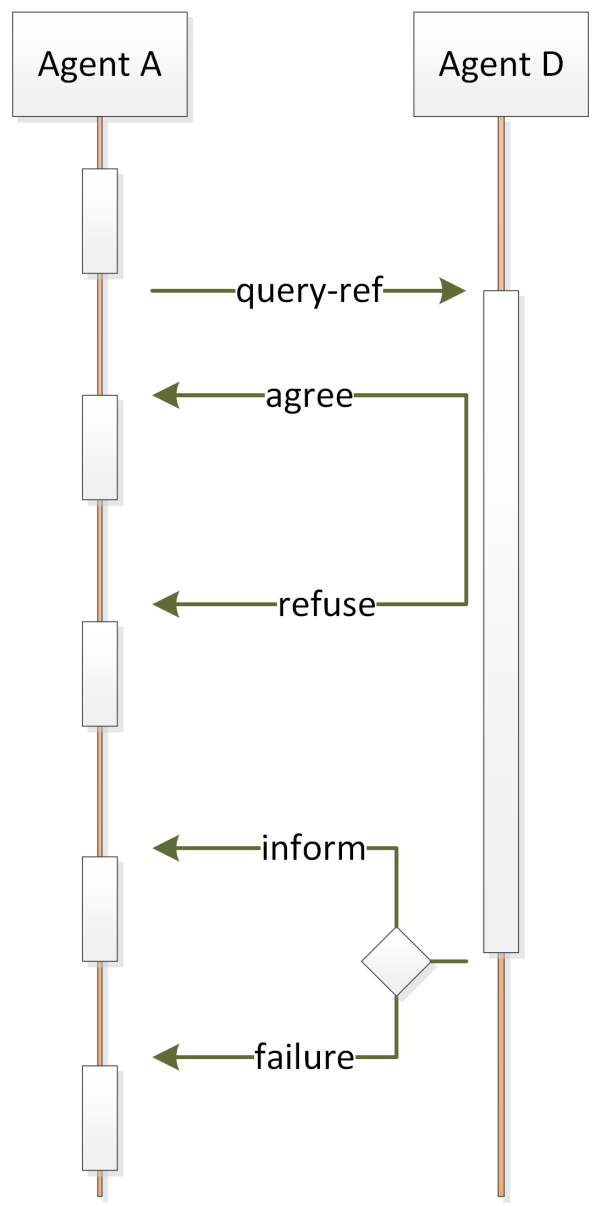

Figure 3.7: Agent interaction diagram SHowing the Protocol FOR QUERY-REF COMmUnicATIVE $\mathrm{ACT}$ 


\section{Chapter 4}

\section{Fault Location \& Isolation}

A key aspect of self-healing when applied to PDS is the need to identify the fault location. The essential characteristics of improved fault management are:

\section{First}

Quicker detection that an outage has occurred.

\section{Second}

Accurately determining the location of the fault.

\section{Third}

Isolation of the faulted section of a feeder.

\section{Forth}

Re-energizing the un-faulted sections of the feeder outside the isolation zone, upstream and/or downstream of the faulted section.

By fast locating and isolating the faulty zones, ZAs can notify the corresponding FAs to start the restoration process such that the degree of damage can be reduced. The MAS technology makes it possible for the agents to freely exchange information; accordingly, improving the accuracy and quickness of the fault location and isolation [60].

The state-of-the-art of the employed method in this dissertation is the distributed fault location and isolation strategy by ZA's which use their current and voltage phasors as well as their neighbors. 


\subsection{Fault Location Equations}

Each agent has access to its neighbor ZA's data and can calculate the current injected to area between itself and upstream or downstream agents by using the Kirchhoff's law, i.e.

$$
I_{Z_{k}}=\sum_{i=1}^{n} I_{\text {Enterance }}-\sum_{i=1}^{m} I_{\text {Exit }}
$$

where $I_{Z_{k}}$ is the current usage of zone $k$, defined as the difference between the sum of currents entering the zone $\left(I_{\text {Enterance }}\right)$ and currents leaving the zone $\left(I_{\text {Exit }}\right)$. ZAs communicate with their direct neighbors regularly (every 5 mins) and update this value. By monitoring the trend for $I_{Z}$ changes, each $\mathrm{ZA}$ can determine whether there is a fault in its zone or not. Changes are calculated using

$$
I_{Z_{C}}=\left(\frac{\left|I_{Z_{\text {new }}}\right|-\left|I_{Z_{\text {old }}}\right|}{\left|I_{Z_{\text {old }}}\right|}\right) * 100
$$

\subsection{FLI Algorithm}

Fig. 4.1 shows the fault location, isolation algorithm flowchart. In normal operation, agents update their load profile data and also update their neighbor's data. Agent's pull out the meters recorded data during the last recloser trial with highest resolution and compare the data with recent normal operation data.

As a completely decentralized approach, ZAs just have access to local information and they have no information about the topology. The following algorithm specifies the fault location and isolation steps for ZAs.

(1) Each ZA is aware of its current usage using equation (4.1) and updates it sequentially along informing its neighbors.

(2) When there is a fault in the feeder and recloser is going for its three trials, FA informs the ZAs to be aware of the trials.

(3) ZAs pull out the higher resolution measured data during the recloser trials period and update their drawn current value using equation (4.1).

(4) After the recloser locks out, agents exchange the recent measured data during trials with their neighbors. 


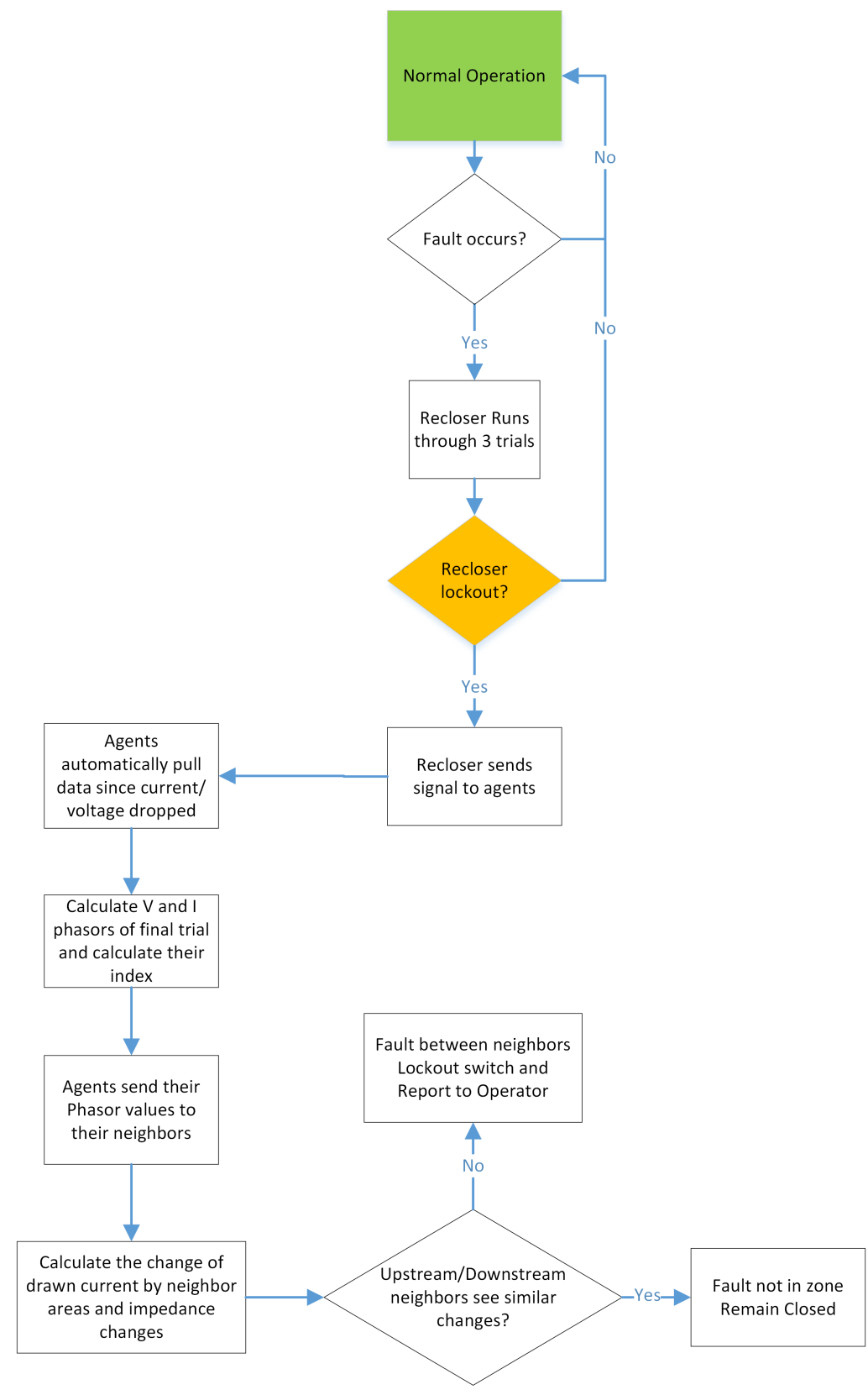

Figure 4.1: FAULT LOCATION, ISOLATION ALGORITHM FLOWCHART

(5) Next, Using equation (4.2) ZAs analyze the changes in their drawn current before and during the recloser trials.

(6) The $Z A_{i}$ with fault sees the highest changes in the index, while other zones either see negative or much lower percentage changes in their load profile.

(7) $Z A$ starts communicating to the downstream ZA to confirm isolating the fault. 
(8) This process is stopped by $Z A_{i}$, when it confirms with its next neighbor $\left(Z A_{i+1}\right)$ and knows that the fault is between itself and its downstream neighbor.

After isolation of the faulted zone, all other feeder zones will disconnect. In this way, they can be recovered one by one and restoration constraints can be better satisfied.

\subsection{Fault Classification}

Faults such as Low Impedance Fault (LIF), High Impedance Fault (HIF) and InRush Current (IRC) are the potential fault types in power distribution systems. An autonomous MAS operation and control strategy can improve the accuracy and quickness of fault location and isolation in dealing with these faults.

If a fault is a low impedance fault (LIF), proposed MAS may accurately detect the fault because of changes in phase current and indices given in (4.2). Generally, in case of ground faults, all the agents between the fault position and recloser experience the fault current and the fault phase might experience low voltage too. The changes in $I_{Z}$ for the faulty zone will be larger than fault-free zones. In case of phase to phase faults, the voltages may show close to 180 degree phase difference with one another, which can be varied depending on loop load impedance.

If the fault is a high impedance fault (HIF), the recloser and other protective devices might not detect the fault since the fault current is smaller than the load current, although it depends on the fault impedance value. HIF can be distinguished from the load currents using harmonic analysis and the wavelet transform [30].

When the fault zone location and type of the fault are determined, switches of the fault zone can be opened to isolate the fault zone and after informing the FA, FA can use the restoration methodology to transfer the fault-free zones to the other feeders. 


\subsection{Fault Location Results}

In order to test the operation of the MAS and power system and to evaluate its performance, different fault scenarios were simulated and the results are presented in this section.

\subsubsection{Impedance Analysis}

\subsubsection{Case I: phase to ground fault between switch 2 and 3}

At time 20 second a single phase to ground fault occurs between switch 2 and 3 according to Fig. 3.4 The recloser detects the over current and locks out. During the three recloser operations the data are communicated among the agents and the agents detect the fault type and zone based on changes in current and impedance values change. Fig. 4.2 shows the recloser operation after fault occurrences. Fig. 4.3 and Fig. 4.4 show the current and impedance change from recloser point of view.

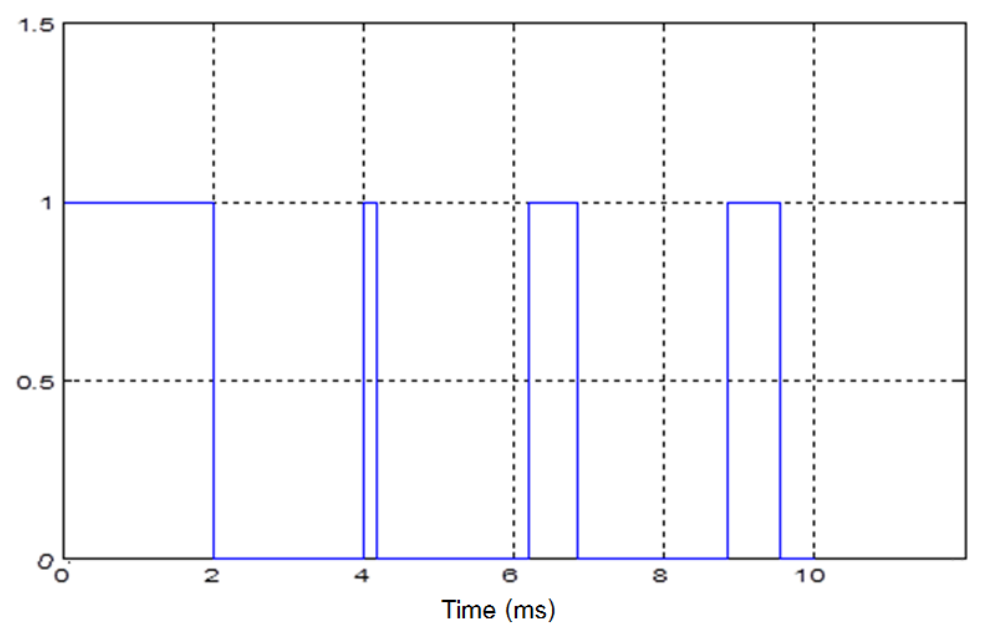

Figure 4.2: RECLOSER OPERATION

In this case, impedance values for the switches after the switch 3 in feeder WR3 does not change after fault occurrences and by this way agents know that the faults is not downstream the switch 3 . The difference between the input and output current of zone 1 where the fault occurs is different and it's another criterion which agents use to locate the fault. 


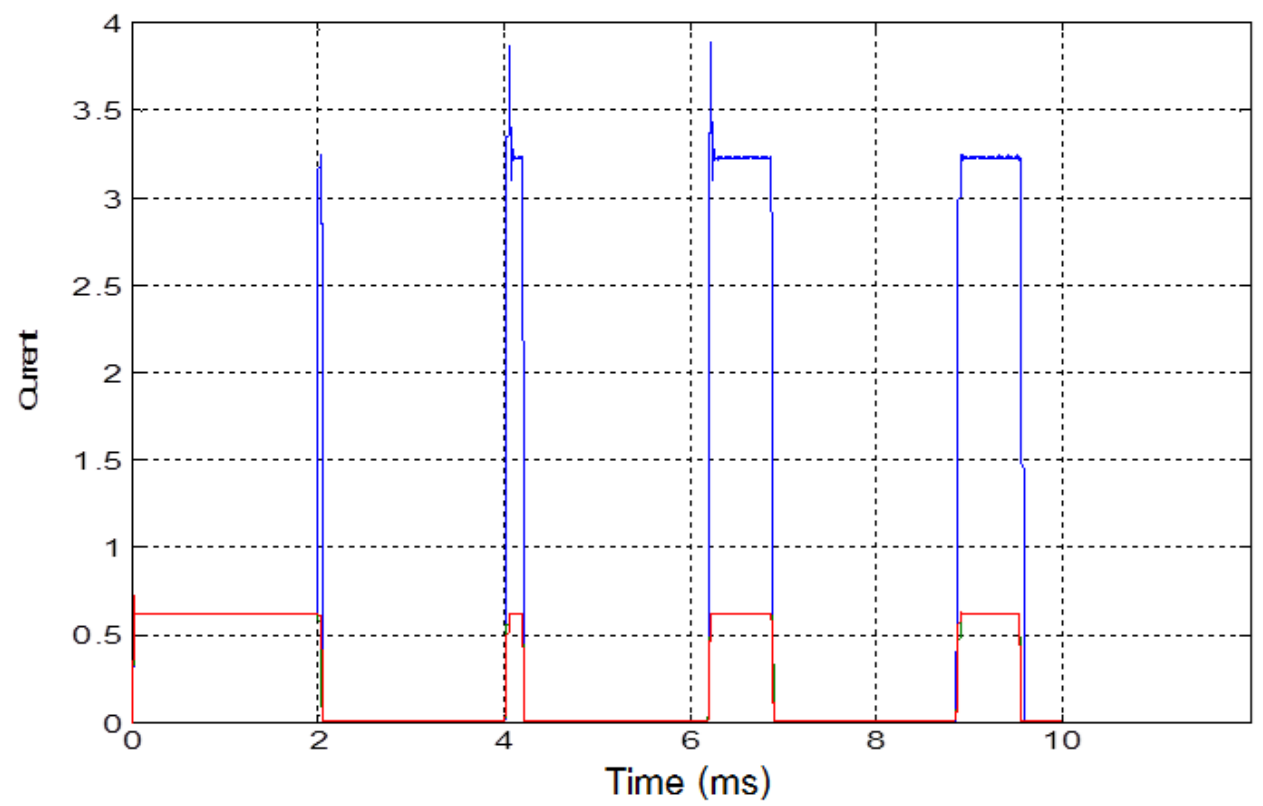

Figure 4.3: Current Changes for Switch 1 During and Before fault (Per unit)

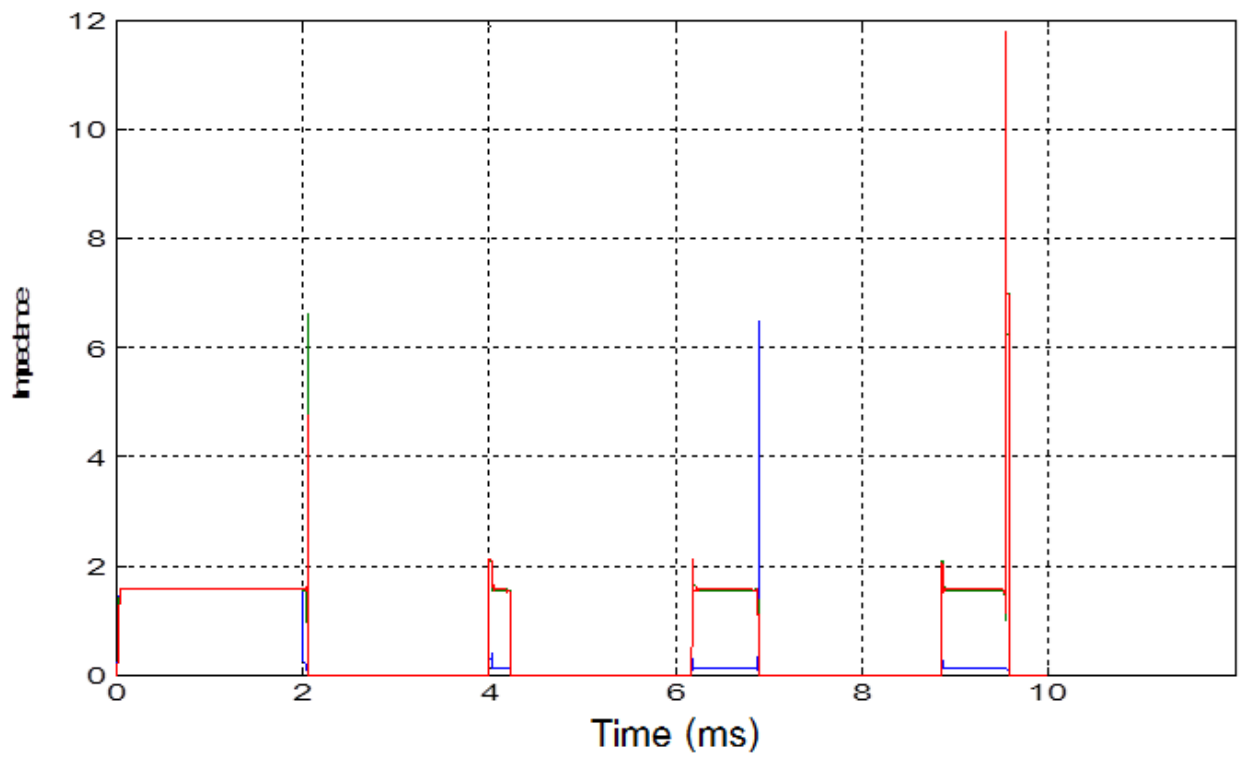

Figure 4.4: Impedance Changes For SWitch 1 DURing ANd Before Fault (Per Unit)

\subsubsection{Case II: phase to phase fault between switch 2 and 3}

A phase A to phase B fault occurs at the time 20 second and Fig. 4.5 and Fig. 4.6 show the current and impedance changes before and after the fault and during the recloser operations. In this case both phase A and B current increased and the impedance decreased. 


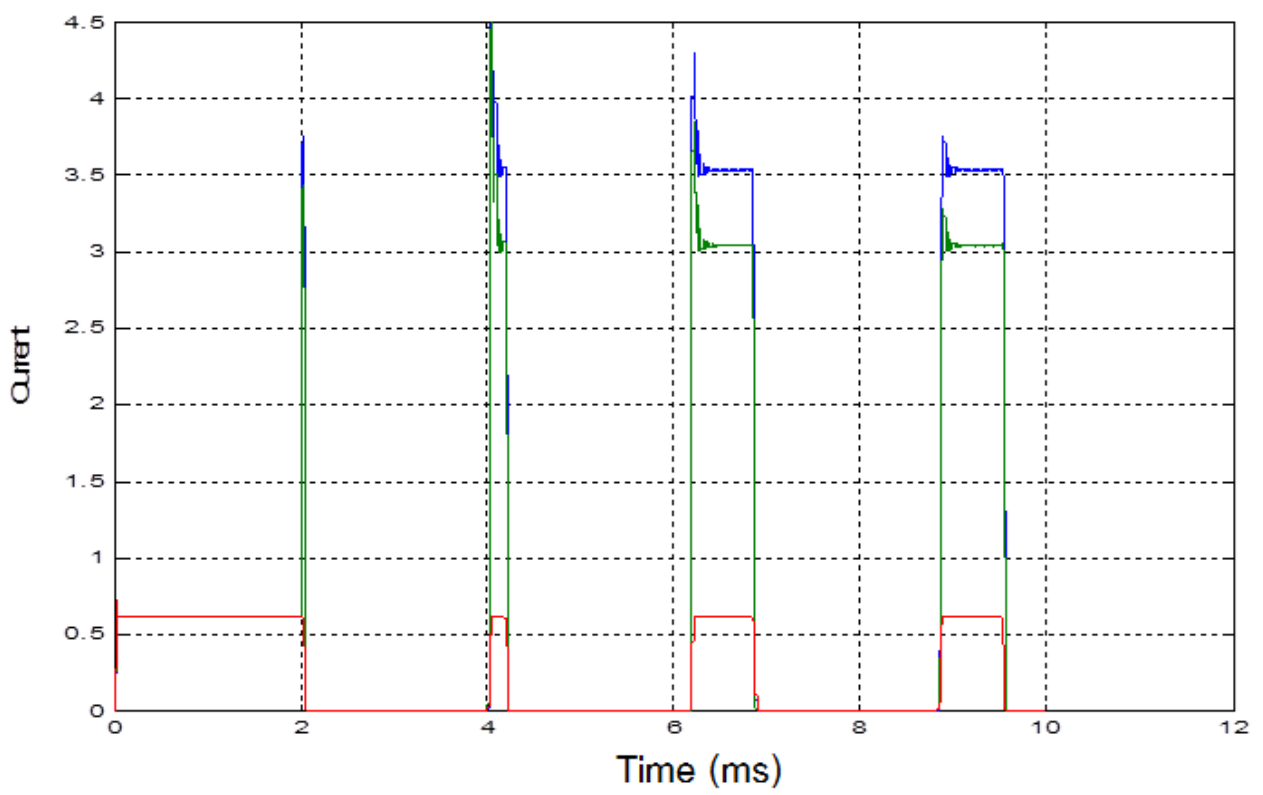

Figure 4.5: Current changes for Switch 1 During and Before fault (Per unit)

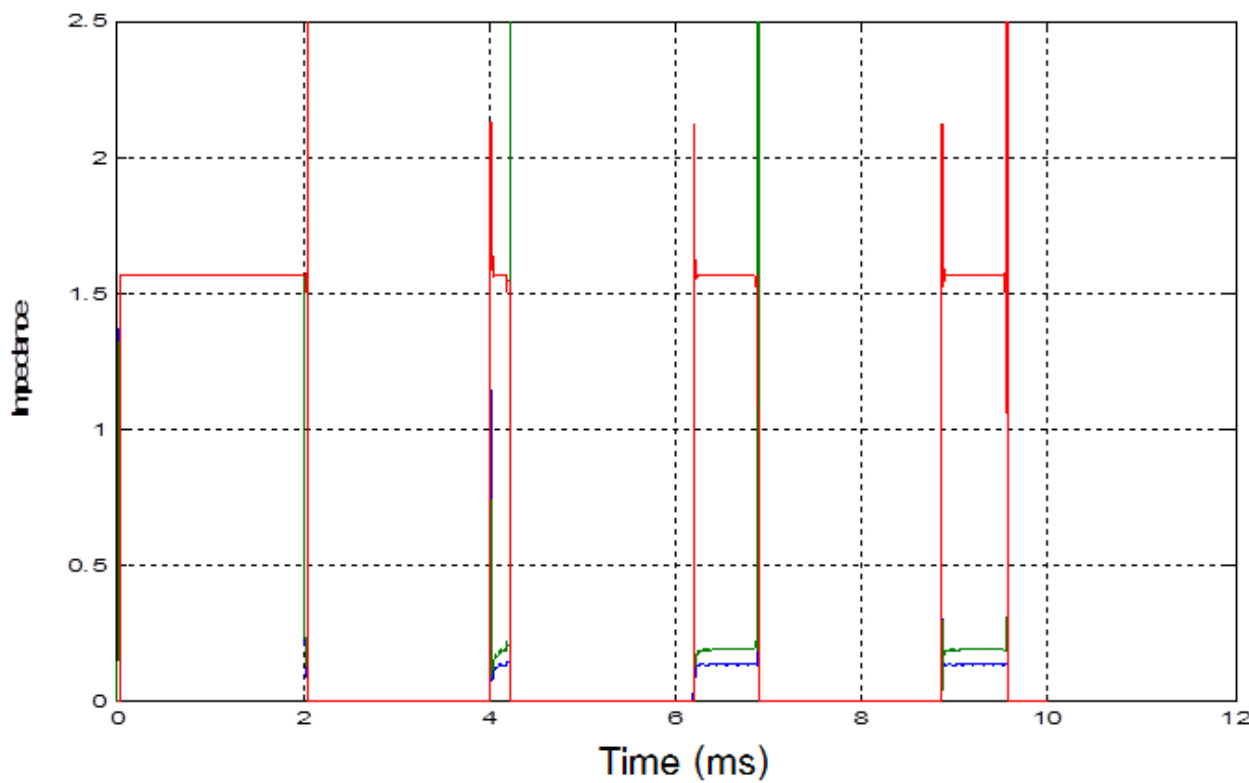

Figure 4.6: Impedance Changes For SWitch 1 During AND Before Fault (Per Unit)

\subsubsection{Proposed Algorithm}

In order to show the effectiveness of proposed fault location, isolation method and investigate the effect of DG sources, different fault scenarios with up to $50 \%$ DG penetration level are simulated. The fault simulation is performed according to the following procedures. First, Each $Z A$ obtains its neighbors zones data every 5 minutes and updates its knowledge about the neighbors. Upon receiving the 
recloser trials, $Z A_{i}$ 's record the data during the trials with highest resolution (16 samples per cycle). Next, Each agent can calculate its zone power usage and inform the neighbors about the updated values. Finally, Each $Z A_{i}$ can decide whether its neighbor zone is the fault zone or not. Here, Faulty $Z A_{i}$ initiates the fault isolation and asks the neighbor $Z A_{i+1}$ to open. At this time, switches at both side of the fault are open and $Z A_{i}$ informs the corresponding $F A$ that the fault is isolated, so that $F A$ can start the reconfiguration and restoration.

Table 4.1 summarizes the simulation results for some fault scenarios. The LG- $a$ field represents the line to ground fault for phase $a$, the LL- $a c$ field the line to line fault between phases $a$ and $c$. In the last column, $S W-i$ shows the number of switches that need to be opened to isolate the fault.

Table 4.1: Fault Location \& Isolation Simulation Results

\begin{tabular}{|c|c|c|c|c|c|c|c|c|}
\hline \multirow{2}{*}{$\#$} & \multicolumn{2}{|c|}{ Fault } & \multicolumn{2}{|c|}{ DG Penetration } & \multicolumn{3}{|c|}{$I_{Z_{C}}(\%)$} & \multirow{2}{*}{ Sw's to Open } \\
\cline { 2 - 9 } & Type & Location & $\%$ & Location & $\mathrm{a}$ & $\mathrm{b}$ & $\mathrm{c}$ & \\
\hline \hline 1 & LG- $a$ & Zone 1 of F3 & 0 & - & 90 & -65 & -65 & SW1 \\
\hline 2 & LG- $a$ & Zone 1 of F3 & 50 & Zone $2 \& 5$ & 60 & -35 & 40 & SW1 \\
\hline 3 & LG- $c$ & Zone 3 of F3 & 0 & - & -50 & -65 & 150 & SW3 \& SW5 \\
\hline 4 & LG- $c$ & Zone 3 of F3 & 50 & Zone $2 \& 5$ & -15 & -50 & 120 & SW3 \& SW5 \\
\hline 5 & LL- $a c$ & Zone 3 of F3 & 50 & Zone $2 \& 5$ & 200 & -100 & 600 & SW3 \& SW5 \\
\hline 6 & LG- $a b c$ & Zone 2 of F3 & 50 & Zone $2 \& 5$ & 400 & 1400 & -100 & SW2 \& SW3 \\
\hline
\end{tabular}

The effectiveness of the proposed method can be identified by comparing the $I_{Z_{C}}$ field shown in Table 4.1. The $I_{Z_{C}}$ index increases the most for the faulty zone and in the other zones the index is decreased or not changed much. This means that the $Z A$ experiencing the fault can accurately determine whether the fault had occurred in its zone or not.

Fig. 4.7 shows the calculated I zone for zones 1, 2 and 3 while a single line to ground fault is located in zone 2 and without the DG penetration. Fig. 4.8 shows the results for the same scenario but with the $50 \%$ DG penetration. In this scenario DGS are located in zone 2 and 4 . The red line shows the faulty phase.

The I-zone index just increased in the faulty zone and in the other zones the index is decreased in both cases with or without DG generation. Fig. 4.9 shows the I-zone index for DG penetration of $50 \%$ with a line to line fault in zone 2 . I-zone 

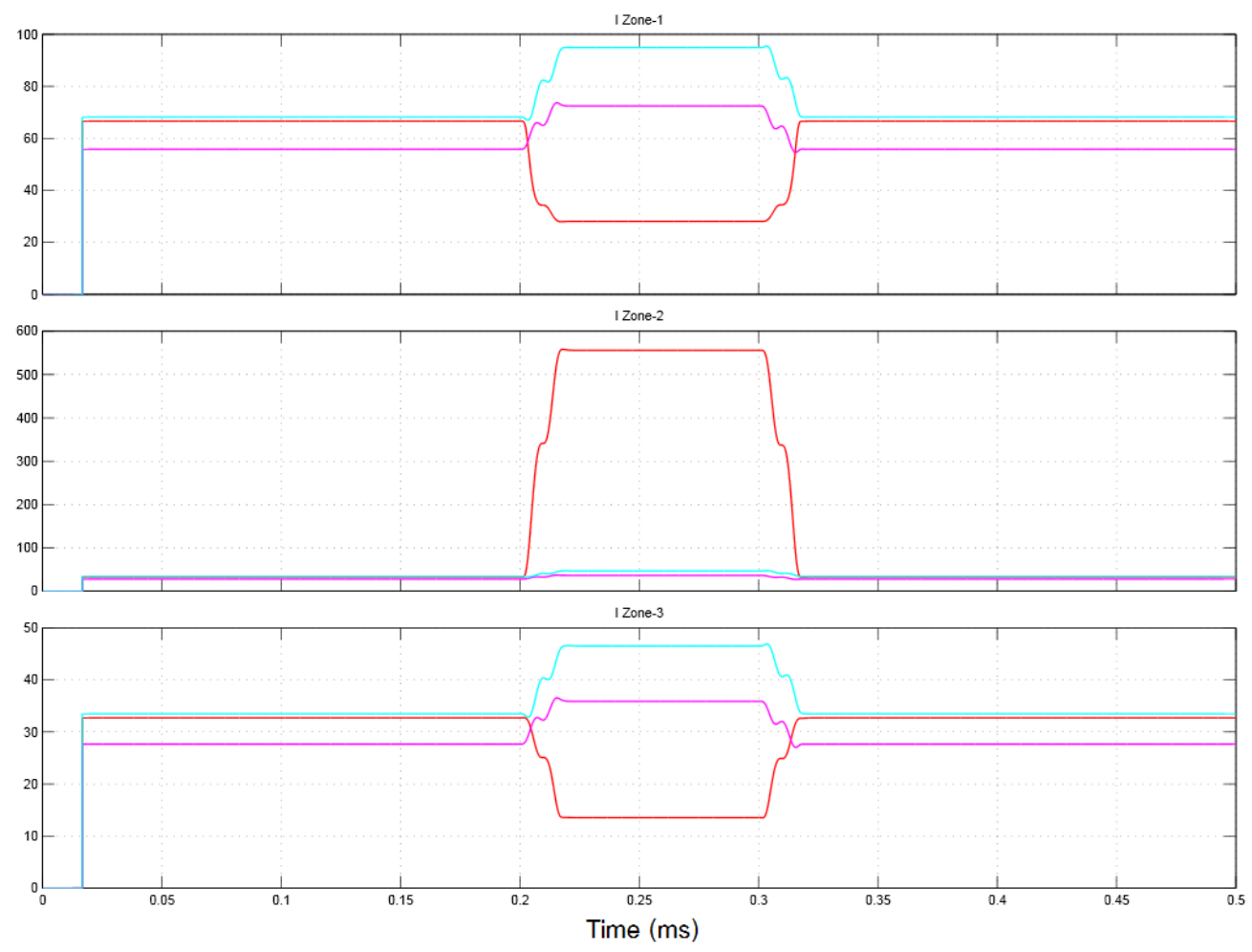

Figure 4.7: Calculated index for DG Penetration of 0\%

index increased in both zone 1 and 2, but change percentage in zone 2 is a lot greater that the zone 1 . 

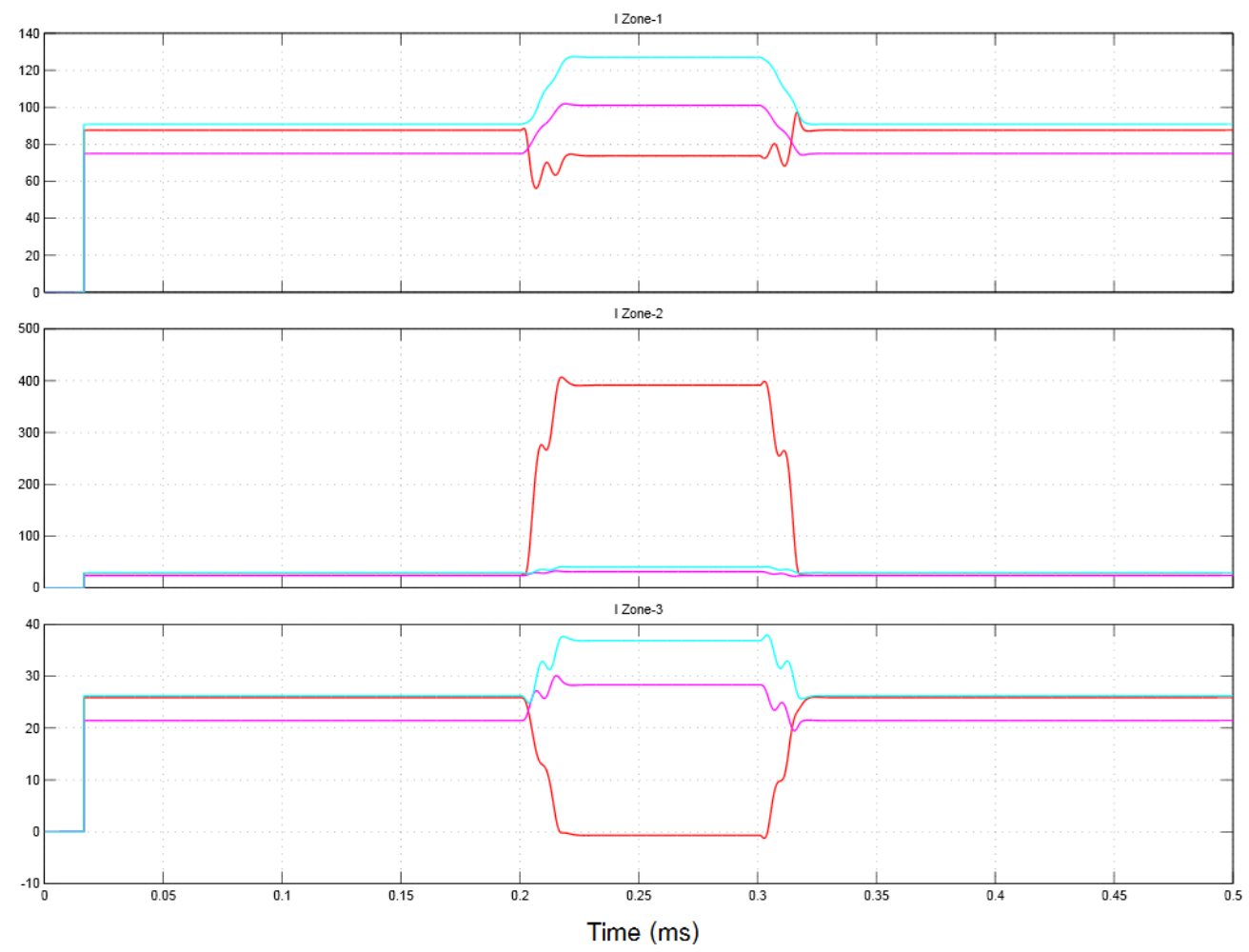

Figure 4.8: Calculated index for DG Penetration of $50 \%$
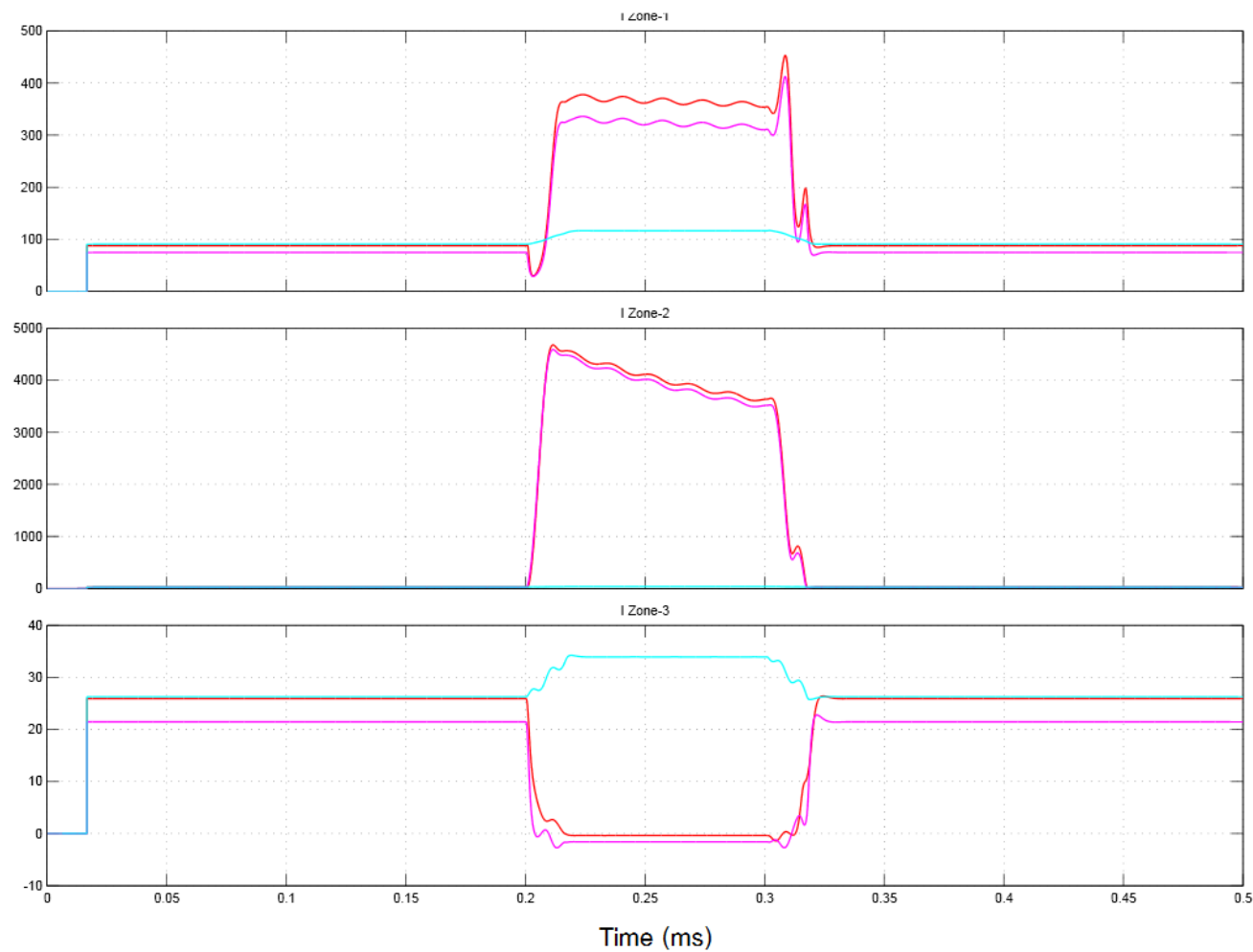

Figure 4.9: Calculated index For DG Penetration of 50\% 


\section{Chapter 5}

\section{Service Restoration}

Service restoration is one of the distribution automation (DA) functions which represent the cornerstone for smart grid development strategy. Service restoration is defined as finding a suitable backup feeders and laterals to transfer the loads in out-of-service areas using operational criteria through a series of switching operations. Performing the restoration task under emergency situations makes it time limited and a complex problem. Self-healing represents the heart of the smart grid. Self-healing can be described as a system that when subjected to a fault, it will be able to automatically and intelligently perform corrective actions to restore the system to the best possible state, thus enabling it to perform its basic functions. Therefore, the ability to quickly and flexibly reconfigure the network to restore the de-energized loads by the fault represents the key component of self-healing function. Key Benefits of the automatic service restoration are:

- Increase network reliability: by proactively monitoring the grid for potential problems.

- Enhance operational efficiency: by managing restorations electronically and reducing the need for radio communications.

- Improve customer satisfaction: by reducing the time without power for customers.

- Lessen operational costs: by quickly determining the exact fault zone and reducing the crew's traveling time along the feeders. 
- Reduce time to restore power: by using the FLIR algorithms and faster fault location, isolation and service restoration.

After isolation of the fault by ZAs and once total net power and demands of fault free zones required for restoration are obtained, corresponding FA uses mathematical optimization technique to evaluate the alternative reconfigurations.

\subsection{Restoration Formulation}

The restoration problem is formulated as 0-1 Knapsack Problem, which is a NPhard combinatorial optimization problem. The mathematical formulation of the problem at state $s$ is shown below with its objective function and constraints.

Objective Function:

$$
\operatorname{Max}\left\{J(s)=\sum_{i=1}^{n} \gamma_{i}\left(\rho_{i a} \cdot I_{i a}+\rho_{i b} \cdot I_{i b}+\rho_{i c} \cdot I_{i c}\right)\right\}
$$

where $I_{i a}, I_{i b}$ and $I_{i c}$ represent the load current corresponding to phases $a, b$ and $c$ of $i^{\text {th }}$ zone, and $\rho_{i}$ is the weight associated with each zone load, depending on its priority. $\gamma_{i}$ is binary value determining whether the $i^{\text {th }}$ zone should be restored or not (1: restored; 0: not restored).

The fulfillment of the objective should be obtained by satisfying the following constraints.

Constraints:

1. Zone Constraint: The sum of flows into any zone should be equal to the sum of flows leaving the node, plus the zone's load current.

$$
\sum_{i \in \text { in }_{i}} I_{i-f}=\sum_{i \in \text { out }_{i}} I_{i-f}+I_{i f}
$$

where $\mathrm{f}$ is the phase $(a, b$ and $c)$. $i n_{i}$ and $o u t_{i}$ are the set of input and output current flows into and out of zone $i$. $I_{i f}$ represents the current drawn by zone $i$ itself. 
2. Source Capacity Constraint: The sum of the flows going out of a zone should not exceed the total available capacity of the respective zone.

$$
\sum_{i \in \text { out }_{i}} I_{i-f} \leq I_{\text {cap }-i f}
$$

where $I_{c a p-i f}$ is the available capacity in phase $f$ of $i^{\text {th }}$ zone.

3. Radial configuration Constraint: The radiality constraint ensures that only one branch feeds each zone.

$$
\sum_{i \in i n_{i}} S_{i-f} \leq 1
$$

where $S$ is the status of switches at the feeding side of the zone and is defined as follows: $S_{i}=1$ if switch $i$ is closed and $S_{i}=0$, otherwise.

4. Voltage Drop Constraint: Voltages at end of zones should be within standard limits. The voltage drop is calculated by the $K-d r o p$ value

$$
K_{\text {drop }}=\frac{\text { Percent_Voltage_Drop }}{k V A \_ \text {mile }}
$$

$K$ - factor is the voltage drop percent down a line that is one mile long and serving a balanced three-phase load of $1 k V A$. $l$ is the one way distance and $k V A$ is the actual load of the line [61].

The voltage drop along a line segment is computed by:

$$
V_{\text {drop }}=K_{\text {drop }} \cdot k V A \cdot \text { mile }
$$

Voltage drop should be within the limits i.e. $V_{d} \leq 5 \%$. These constraints will ensure that while optimizing the restoration, the voltage constraints are also satisfied.

\subsection{Illustration}

The restoration procedure may be initiated by the faulted FA when it receives the fault location and isolation information from corresponding ZAs. As a result, the 
system may be split into two sub-systems, fault upstream and downstream zones. First, FA restores the zones upstream the fault, one by one. Next, to restore the downstream zones, FA searches for the alternative restoration paths using either its learned knowledge or solving the restoration optimization technique for current state. If the learning knowledge is adequate for restoration decision making, FA makes the decision and starts communicating to target feeders. Otherwise, It solves the restoration optimization problem to obtain the optimal post-fault configuration.

To solve the optimization problem, FA sends request for proposals to the neighbor feeder agents which have the capability of transferring power to fault downstream zones through tie switches. Feeder agents are aware of their zones power demand and can respond to a proposal if they can support additional loads that are to be transferred. Once FA receives the net power of fault-free zones that are ready for restoration and also neighbor FA's extra available power, an optimal load restoration decision can be obtained by satisfying the restoration constraints ((5.2)-(5.4) and (5.6)). Once the zones that can be restored are identified, load restoration process can be started. During load restoration process, zones will be restored one by one by closing the associated switches.

\subsection{FLIR Flowchart}

Before introducing the FLIR algorithms and learning approach, we describe the steps followed for the healing of the PDS after a fault occurrence. Fig. 5.1 shows a flowchart of the FLIR and learning processes. After the fault location and isolation, the zones upstream to the fault are restored first. Then the feeder agent of the faulty zone goes through the learning database and looks for the related experiences. If FA had enough knowledge about the situation and was confident about the learning solution, it specifies the target reconfiguration. Otherwise, FA goes through restoration optimization and tries to find the optimal reconfiguration. Next, FA knows the target configuration and sends a proposal to the determined feeder agent which has the capability of transferring power to faulted downstream zones through tie switches. Feeder agents are aware of their zones power demand profile and can accept a proposal if they can support additional loads that are to be transferred. If the proposal gets accepted, FA starts to initiate the reconfiguration to restore the out of service zones. After restoration, FA checks the restored zones 
from voltage point of view and isolates them if restoration goal had not been achieved. In this method, since agents are updating their knowledge about their environment frequently (every 5 mins), they are aware if other agents have extra power. Therefore, FA just negotiates with one potential service provider at a time, which consumes less time and fewer number of communication messages. 


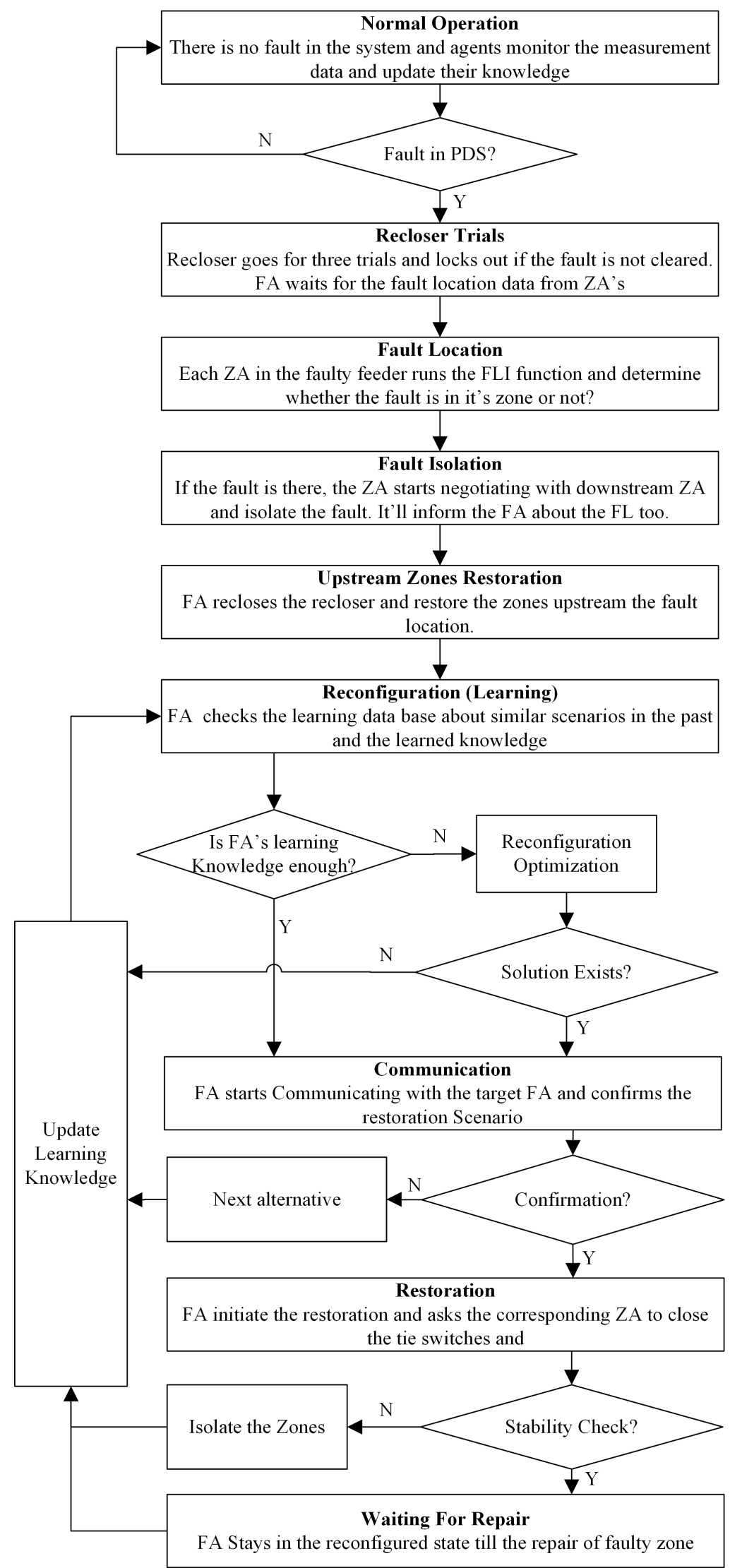

Figure 5.1: FlowChart OF SELF-HEALING FLIR with LEARNING OF PDS 


\section{Chapter 6}

\section{Learning Methodology}

\subsection{Motivation}

Although the agents in a multiagent system can be programmed with behaviors designed in advance, it is often necessary that they learn new behaviors online, such that the performance of the agent or of the whole multiagent system gradually improves. This is usually because the complexity of the environment makes the a priori design of a good agent behavior difficult, or even, impossible. Moreover, in an environment that changes over time, a hardwired behavior may become inappropriate.

\subsection{Reinforcement Learning (Q-Learning)}

In this chapter, the necessary background on multi-agent Reinforcement Learning $(\mathrm{RL})$ is introduced. A reinforcement learning agent learns by interaction with its dynamic environment. At each time step, agents perceive the environment state and take an action based on the observations, which causes the system to transit into a new state. After transition and perceiving the new state, agents reward or punish their actions based on the quality of transition. Q-Learning algorithm is a reinforcement learning algorithm proposed by Watkins to deal with Markov Decision Problems (MDP) [50]. It is extensively used to solve the strategy control and learning problems of autonomous agents. 
RL usually can be described as a Markov decision process, in which an agent must choose the sequence of actions that maximize some reward-based optimization criterion [62]. Agent objective is choosing its action in a way that maximizes the reward value function:

$$
V\left(s, \pi^{*}\right)=\max _{a}\left\{r\left(s, s^{\prime}, a\right)+\gamma \sum_{s^{\prime}} P\left(s^{\prime} \mid s, a\right) V\left(s^{\prime}, \pi\right)\right\}
$$

Where $r\left(s, s^{\prime}, a\right)$ is the reward for taking action $a$ at state $s$ for transition to the next state i.e. $s^{\prime} . P\left(s^{\prime} \mid s, a\right)$ is the probability of transiting to state $s^{\prime}$ after taking the action $a$ in state $s$. and the scalar $\gamma$ is the discount factor $(0<\gamma<1)$. For any finite MDP, there is at least one policy $\pi^{*}$ (optimal solution) that satisfies the following equation i.e.

$$
V\left(s, \pi^{*}\right) \geq V(s, \pi)
$$

Such a policy is an optimal policy, and the corresponding value function is the maximum value for state s. The famous $Q$-function or $Q$-Value can be written as:

$$
Q(s, a)=r\left(s, s^{\prime}, a\right)+\gamma \sum_{s^{\prime}} P\left(s^{\prime} \mid s, a\right) V\left(s^{\prime}, \pi\right)
$$

By this equation, $Q(s, a)$ is the total discounted reward of taking action $a$ in state $s$ and following the optimal policy thereafter. The object of this is to look for the policy which can maximize the reward in future. Comparing equations 6.1 and 6.3 we have,

$$
V\left(s, \pi^{*}\right)=\max _{a} Q^{*}(s, a)
$$

Where:

$$
Q^{*}=\max _{a} Q(s, a)
$$

If we know the $Q^{*}(s, a)$, we can find the optimal policy to take under any state. Therefore, the problem is finding the $Q$-values for each state. 


\subsubsection{Learning}

In the $Q$-learning algorithm, the state-action value or $Q$-value associated with every possible state $s$ and each action $a$, is maintained. Assuming agents start with arbitrary initial values for $Q(s, a), Q$-learning allows us to approximate the $Q$-value from empirical examples obtained from the actual experiences. The updating rule is:

$$
Q_{t+1}\left(s_{t}, a_{t}\right)=(1-\beta) Q_{t}\left(s_{t}, a_{t}\right)+\beta\left[r_{t}+\gamma V\left(s^{\prime}\right)\right]
$$

Where $\beta[0,1)$ is the learning rate parameter which determines how much the new experiences affect the old $Q$ value and is typically time varying, decreasing with time. Authors in reference [63] proved that the sequence generated by equation 6.6 converges to $Q^{*}$ if all states and actions have been visited often and the learning rate satisfies certain constraints.

\subsubsection{Exploration}

There are different action selection mechanisms [64] which select the actions based on the $Q$-values. The $Q$-values, $Q(s, a)$ of any given state reflects the effectiveness of the action, with higher values corresponding to better actions. Thus under a greedy policy, the best action to apply to a given state is simply

$$
a=\operatorname{argmax}^{\prime}\left\{Q\left(s, a^{\prime}\right) \cdot h\right\}
$$

Where $h$ is duration of extra-power availability (hour). As an immediate consequence of this greedy strategy, actions that are chosen in such a manner that loads with higher rated capacities are likelier to be supplied with power. This is a desirable outcome, as the final sequence of operations tends to deliver power to larger vital loads first. Unfortunately, the greedy rule can obtain the best actions after the algorithm has explored a sufficient number of possible configurations and converged towards the actual $Q$-values. In order to explore the effectiveness of other actions during the learning stages of the algorithm, action selection must be carried out in a probabilistic manner. Under these circumstances, the probability of an action being taken from a state is given by 


$$
\operatorname{prob}(a \mid s)=\frac{\exp \left(-\frac{Q(s, a)}{\tau}\right)}{\sum_{a^{\prime}}\left(-\frac{Q\left(s, a^{\prime}\right)}{\tau}\right)}
$$

The quantity $\tau$ is called the temperature. When $\tau$ is high, all actions are almost equally likely. When $\tau$ is lowered the probabilistic strategy becomes increasingly similar to the greedy strategy. Therefore, in order to make the algorithm exploratory, $\tau$ is initialized with a high value $\tau_{0}$ and decreased gradually towards a final low value $\tau_{\infty}$. In each iteration, the $Q$-learning algorithm applies the probabilistic strategy of (6.8) to combine exploratory search with exploitation. At the end of the predetermined number of iterations, the greedy strategy is applied to obtain a minimal sequence of switching operations necessary to restore the maximum possible power.

\subsection{Restoration Learning Approach}

The object of the Q-learning is to look up among the experienced restoration scenarios to find a strategy to restore the power to as many loads as possible. This can be achieved using Q-learning for restoration which consists of policy bank module, policy trust module, Q-update module and reward computation module as shown in Fig. 6.1. The function of each module is described in the rest of this section.

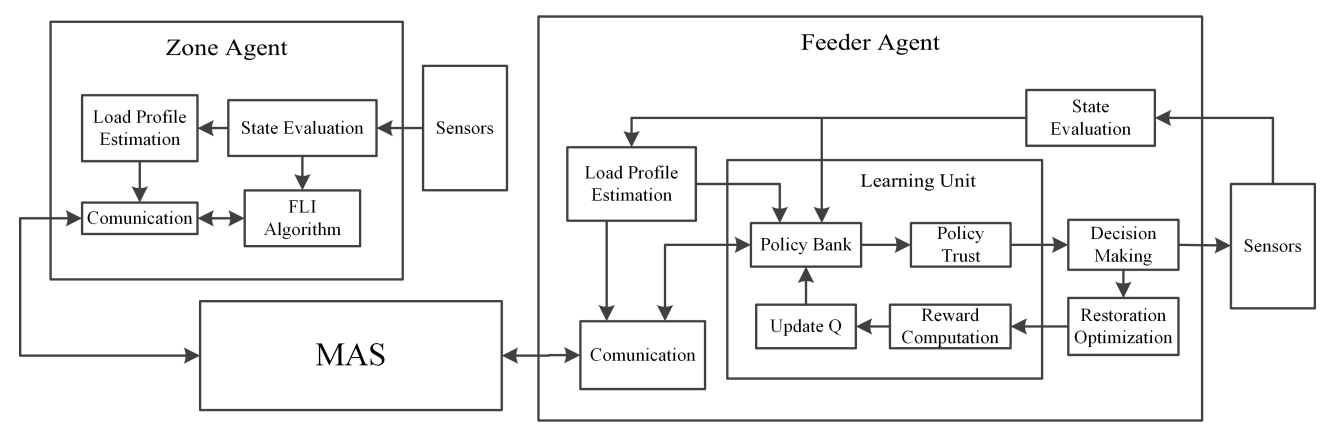

Figure 6.1: LeArning Modules in A FA

The advantage with the Q-learning is that the agents' knowledge will be continually updated online as the system takes action and receive the reinforcement learning signals from power system. In this work we used the offline learning based on simulation models to initialize the $\mathrm{Q}$ values for each scenario. In this 
manner, multi agent system will have a primary knowledge about scenarios it might encounter in the future.

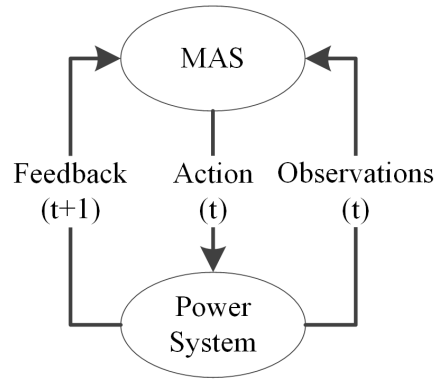

(A)

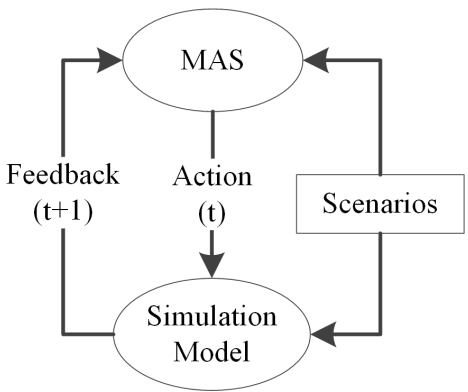

(B)

Figure 6.2: (A) Online And (B) Offline Learning Modes

In offline mode (Fig. 6.2) multi agent system will carry out the simulations about every restoration scenarios not encountered before to obtain a learning knowledge before applying them to the real world power system.

FAs have limited number of possible remedial actions to restore the power to faultfree zones. By Combining all FAs possible states and behaviors, the state set and action set for restoration can be created. Let $s_{k}=\left\{s_{1}, s_{2}, \ldots, s_{m}\right\}$ be the state set, and $a_{k j}=\left\{a_{k 1}, a_{k 2}, \ldots, a_{k n}\right\}$ indicate the possible restoration paths at state $k$. At any state $s_{k}$, any action $a_{j}$ produces a transition to another state, $s_{k}^{\prime}$, the changes in objective function as a result of this transition are used as a part of reinforcement to the $Q$-Learning algorithm.

$$
R(s, a) \propto\left[\Delta J(s, a)=J\left(s^{\prime}\right)-J(s)\right]
$$

In the above equation, $\Delta J(s, a)$ represents the improvement in the objective function if action $a$ is taken in state $s$. In any state, agents take the action that causes the largest increase in the objective function. $F A_{k}$ decides about the restoration strategy according to the accumulated reward values of reinforcement signals for each state, saved into $Q$-values. The reward for taking action $a$ in state $s$ is a function of the changes in the objective function and voltage level after restoration and is calculated by:

$$
R(s, a)=\frac{\Delta J(s, a)}{P_{\text {Outage }}} \cdot\left(1-V_{\text {drop }} \%\right)
$$

Where $P_{\text {Outage }}$ represents the amount of required power to restore the fault-free zones. 


\subsubsection{Q-Matrices}

For a feeder agent, Q-matrix is the repository of all the information learned through its own experiences. FAs can use this matrix data for decision making and see how the past actions worked. The size of the Q-matrix for each feeder agent depends on the number of zones and number of connected feeders with tie switches.

$$
Q_{F A_{x}}\left(s_{k}, a_{i j}\right)=\left[\begin{array}{cccc}
Q_{11} & Q_{12} & \ldots & Q_{1 j} \\
Q_{12} & Q_{22} & \ldots & \ldots \\
\ldots & \ldots & \ldots & \ldots \\
Q_{i 1} & \ldots & \ldots & Q_{i j}
\end{array}\right]
$$

While $i$ is number of zones in $F A_{x}$ and $j$ is number of connected feeders to $F A_{x}$. $0 \leq Q_{i j} \leq 1$ represents the accumulated reward for feeder agent $x$ to ask help from feeder $j$ to restore the power to zone $i$ at the state $s_{k}$.

For any state, there is at least one action $\left(a^{*}\right)$ that has the largest reward among the possible actions for zone $i$ restoration in state $s$.

$$
Q\left(s, a^{*}\right) \geq Q(s, a)
$$

\subsubsection{Q-Update}

During the learning and after transition to any new state the Q-matrix will be updated using a weighted average shown below and the updated Q-matrix will be utilized for action selection in future.

$$
Q_{t+1}\left(s_{t}, a_{t}\right)=(1-\beta) Q_{t}\left(s_{t}, a_{t}\right)+\beta\left(\bar{Q}_{t}\left(s_{t}, a_{t}\right)\right)
$$

Where $Q$ and $\bar{Q}$ are the last updated and the most recent obtained $Q$-values and $\beta$ can be interpreted as the parameter of learning rate. We can make use of changing the learning rate to insure the learning efficiency. Since, the alternation of $Q$-value can reflect the learning progress [62]; we can use the changes in $Q$-value to adjust the learning rate. Therefore; we can define the $\Delta Q$ as follows:

$$
\Delta Q=\frac{\left|Q_{\text {new }}(s, a)-Q_{\text {old }}(s, a)\right|}{Q_{\text {old }}(s, a) \cdot n(s, a)}
$$


Where $n$ is the number of trials for the state $s$ explored in the past. We can conclude that the value of $\Delta Q$ should reduce progressively if $Q$-learning converges and conversely if the $\Delta Q$ is oscillating and diverges. Using this algorithm, we will compare the learning result of its current cycle with the similar forward ones and alter the learning rate in order to learn a convergence result.

\subsubsection{Action Selection Strategy}

In this work, we use deterministic greedy strategy for making action choices; it chooses the superior actions according to the current state, $Q$-matrix values. If there are more than one alternative for restoration, $F A s$ choose the substitute feeders in such a way that maximizes the total rewards i.e.

$$
a=\operatorname{argmaxa}^{\prime}\left\{Q\left(s, a^{\prime}\right) \cdot h\right\}
$$

Where $h$ is the possible duration of restoration. The confidence of $Q$-learning in decision making depends on the $Q$-value for each action. if $Q\left(s, a^{\prime}\right) \geq 0.95$ it means that action $a^{\prime}$ can provide the power to corresponding fault free zone with a voltage drop less than $5 \%$.

This action selection strategy obtains the best action choices if we have explored sufficient number of possible actions. If not, this action selection strategy might stay in a local superior point. Therefore, in this work we explore all possible remedial actions using simulation to prevent the system trapping in local minimas. 


\section{Chapter 7}

\section{Restoration Case Studies and Simulations}

The WVSC (West Virginia Super Circuit) (Fig. 3.4) is used to test the proposed MAS, and the restoration results are compared with similar approaches. The WVSC is simulated by using the SimPower package on MATLAB[65] and also CYME[66] for voltage drop studies, all agents are implemented using the Level-1 user defined functions in Simulink. Since both MAS and power system model are implemented in Simulink, there is no need for an interface between MAS and power system model. This advantage provides a simpler and more accurate simulation model compared to some previous works [17, 25] which developed interfaces between power simulation models and software agents. During the simulation, S-functions use a special calling syntax called the S-function API that enables you to interact with the Simulink engine and synchronize the simulation of agents with power system model. In this chapter after going through two case studies, the performance of proposed approach is analyzed.

\subsection{West Virginia Super Circuit}

There are 16 switches and 2 reclosers installed in these two feeders to enable the system. Switches are Cooper DAS-15 type three-phase vacuum switches with $15 K V, 630 A$ ratings. 7 switches are normally closed and the other 9 switches will be used for reconfiguration and restoration applications. Fig. 7.1 shows the one line diagram for WR-3 and WR-4 feeders of WVSC. Transformers in each feeder 
are 138/12.5 $K V$ and 33.6 $M V A$. Simulation results are calculated based on per unit values. The base $M V A$, voltage values are $6 M V A, 12.5 \mathrm{KV}$.

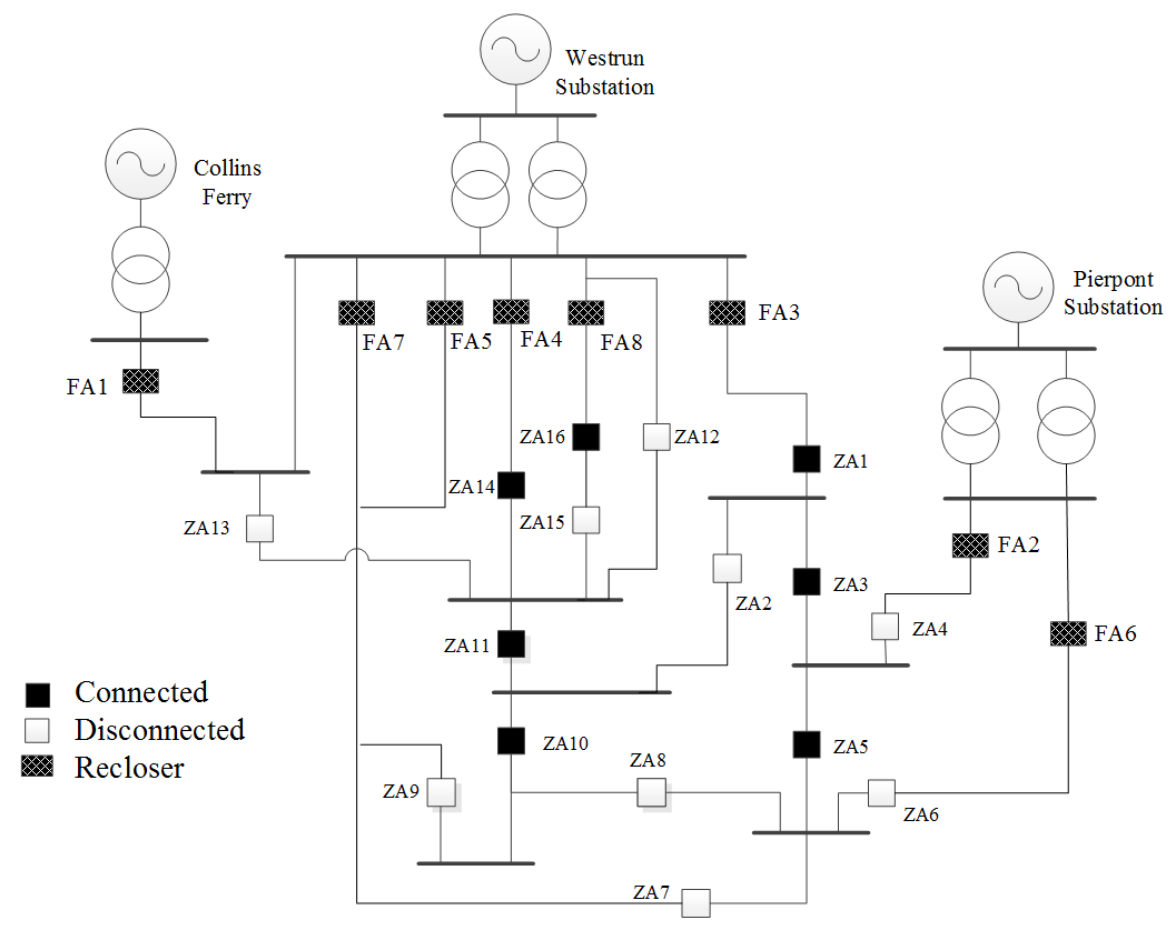

Figure 7.1: The WVSC POWER SYSTEM

Table 7.1 shows the peak load data for each feeder of WVSC.

Table 7.1: WVSC Feeders Peak Load Data

\begin{tabular}{|c|c|c|c|c|c|c|c|}
\hline \multicolumn{2}{|c|}{ Feeder \# } & 3 & 4 & 5 & 6 & 7 & 8 \\
\hline \hline \multirow{3}{*}{ KW \& KVAR } & $\mathrm{A}$ & 1916,746 & 1381,198 & $1395,-132$ & $1036,-14$ & $844,-25$ & 677,133 \\
\cline { 2 - 8 } & $\mathrm{B}$ & 1467,455 & 1667,355 & 1664,26 & $773,-162$ & 1191,167 & 549,69 \\
\cline { 2 - 8 } & $\mathrm{C}$ & 1864,697 & 1667,363 & $1429,-111$ & 1237,85 & $725,-87$ & 442,16 \\
\hline
\end{tabular}

\subsubsection{Recloser Setting}

Time Current Characteristic (TCC) information at two current levels for installed reclosers are given in Table 7.2. These reclosers are programmed to operate three times before locking out. The first reclosing operates on A-curve and the following two reclosings operate on D-curve. The approximate clearing times for A-curve and D-curve are also listed. The reclosers wait for 60 cycles between the reclosing operations. 
TABle 7.2: Recloser Setting

\begin{tabular}{|c|c|c|c|c|}
\cline { 2 - 5 } \multicolumn{1}{c|}{} & \multicolumn{2}{c|}{ A Curve } & \multicolumn{2}{c|}{ D Curve } \\
\hline Current $(A)$ & 2000 & 6000 & 2000 & 6000 \\
\hline Clearing Time & 5 cycles & 3 cycles & 43 cycles & 8 cycles \\
\hline
\end{tabular}

\subsection{Simulation Models}

The simulation model has two parts. The first part is for simulating power system distribution network which is the WVSC by MATLAB Simpower Toolbox. The second part is the multi agent system which is implemented in Simulink by using user defined $S$-functions. In the following both parts are discussed in detail

\subsubsection{Power System Model}

Fig. 7.2 shows the Simulink model for WR-3 and WR-4 feeders. Transformers in each feeder are 138/12.5 KV and 33.6 MVA. Simulation results are calculated based on per unit values. The base $M V A$, voltage values are $6 M V A, 12.5 K V$. A block is designed to generate reclosers operation. Whenever recloser senses overcurrent, it locks out after three operations according to the time periods mentioned in Table 7.2, if the fault is not cleared. Lines are modeled based on the positive, negative and zero sequence impedance values. Different types of faults such as single line to ground, line to line, and three phase faults are modeled with a fault block in Simpower Toolbox and ground resistance is considered to be $0.1 \mathrm{ohm}$. Feeder loads are modeled with active and reactive power.

The power system model could simulate in both discrete or continuous models. Since continuous model is more accurate the power system is modeled using the continuous mode.

\subsubsection{MAS Model}

The multi agent system is implemented using the $S$-function blocks of MATLAB Simulink. Each agent is modeled by an S-function. $S$-functions (systemfunctions) provide a powerful mechanism for extending the capabilities of the Simulink@ environment. An S-function is a computer language description of a Simulink block written in MATLABß. $S$-functions use a special calling syntax 


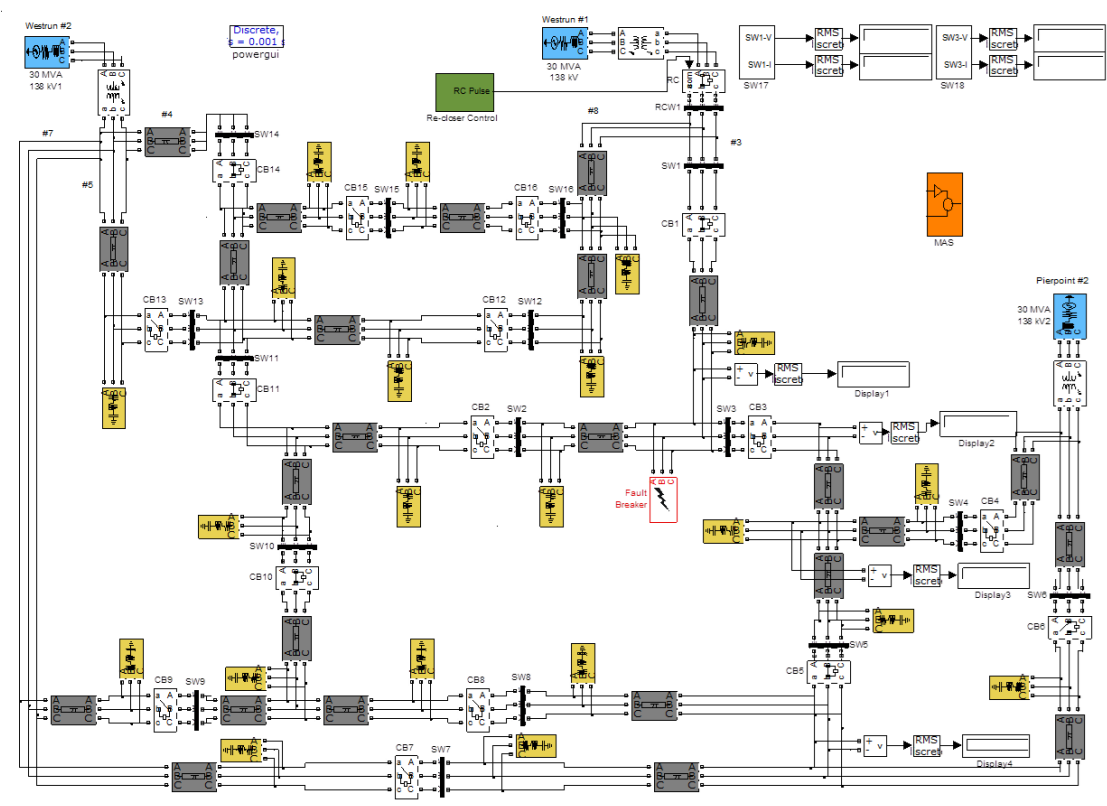

Figure 7.2: Power Distribution System Simulink Model

called the $S$-function $A P I$ that enables you to interact with the Simulink engine. They follow a general form and can accommodate continuous, discrete, and hybrid systems [65]. Fig. 7.3 shows the MAS model.

Since the MAS is mainly including agents communication, and communications are intrinsically discrete in time, MAS works in discrete mode and it also provide the base to model communication delays and latency.

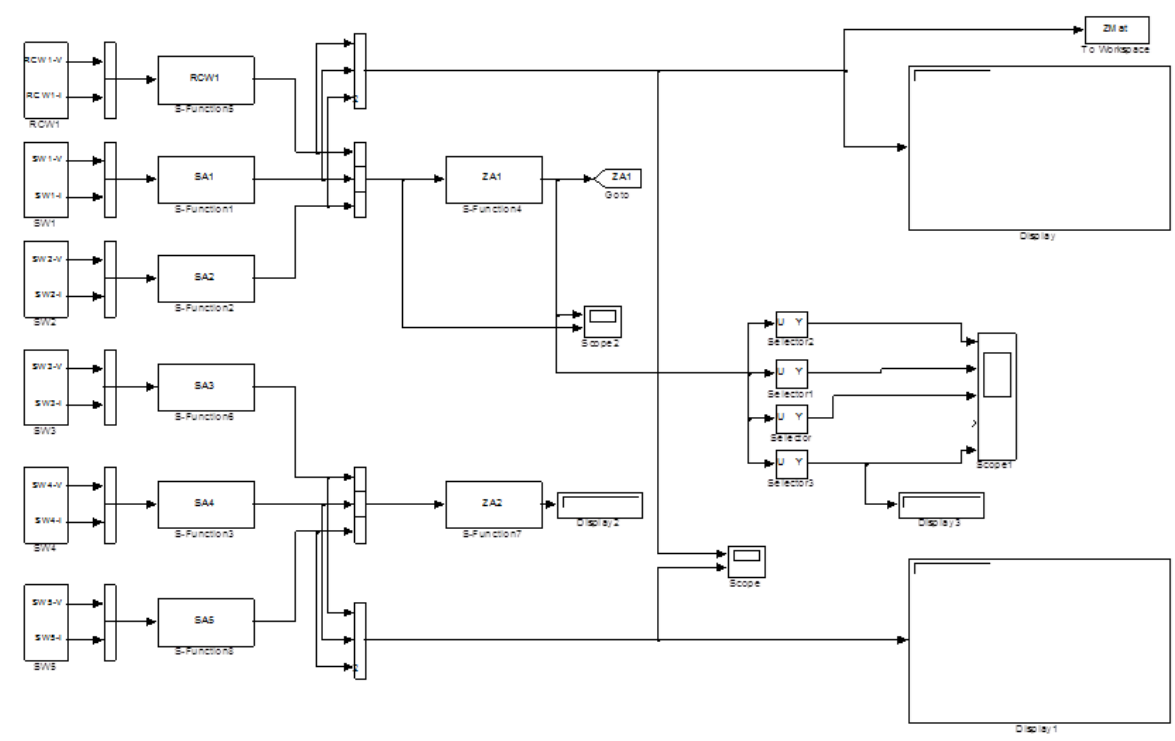

Figure 7.3: Multi agent system model in MATlAB Simulink

Since the measured data resolution is 16 samples per cycle, the agents can access to power system model simulation data with 16 samples per cycle resolution, the 
MAS discrete step simulation time should be 0.001 second. The switch agents have access to voltage and current measurement data and they calculate the impedance value based on the voltage and current rms value. The data packets that switch agents send to their corresponding zone agents have 9 elements. These elements are voltage, current and impedance of each of three phases.

The agents communicate using Foundation for Intelligent Physical Agents (FIPA) standard [58]. Some message performative examples include:

- REQUEST: the sending agent requests the receiving agent to perform some action.

- QUERYIF: the sending agent asks the receiving agent whether or not a given proposition is true.

- INFORM: the sending agent informs the receiving agent that a given proposition is true.

\subsection{Restoration Results}

To verify the effectiveness of the proposed restoration approach, a number of restoration scenarios on the WVSC have been developed under different fault conditions. We are only demonstrating two typical samples: Single phase fault in zone 3 of $F 3$ and phase to phase fault in zone 4 of $F 4$.

\subsubsection{Case I}

A single phase to ground fault occurs in zone 3 of $F 3$. $Z A 3$ and $Z A 5$ isolate the fault and inform $F A 3$ about the fault location. After fault isolation, $F 3$ zones split into upstream zone $(Z 0 \& Z 1)$ and downstream zone $(Z 5)$. Upstream zones will be restored by re-closing the recloser. F3 looks for the alternative restoration path to restore zone 5 using the learning data base or restoration optimization process. The restoration process steps are displayed in Table 7.3.

F3 first tries to find the reconfiguration using the learning knowledge and by using the $Q$-matrix. If $F 3$ finds an action promising with high reward expectation, 
then it starts negotiating with determined $F A$ s to initiate the reconfiguration. Otherwise, $F A 3$ start to solve the restoration optimization problem using the obtained information.

Table 7.3: Proposed MAS FliR procedure (CASE I)

\begin{tabular}{|c|l|}
\hline Step 1 & $F 3$ informs the ZAs before the recloser runs the trials \\
\hline Step 2 & $Z A$ s calculate the introduced indexes \\
\hline Step 3 & analyzing the indexes $Z A 3$ detect the fault \\
\hline Step 4 & $Z A 3$ asks $Z A 5$ to isolate the fault \\
\hline Step 5 & $Z A 3$ and $Z A 5$ lock out and inform $F 3$ \\
\hline Step 6 & $F 3$ restore the upstream zones $(Z 1$ and $Z 0)$ \\
\hline Step 7 & $F 3$ tries to solve the problem using Q-matrix $\left(Q_{F 3}\right)$ \\
\hline Step 8 & $F 3$ decide to asks for help from $F 6$ \\
\hline Step 9 & upon receiving $F 6$ confirmation, $F 3$ asks $F 6$ to close $S W 6$ \\
\hline Step 10 & $F 6$ closes the $S W 6$ and $Z 5$ will be restored \\
\hline
\end{tabular}

Feeder 3 in WVSC is composed of 4 zones and is connected to 4 other feeders through 5 tie switches. $Z 0$ and $Z 1$ will be restored by re-closing the recloser and $Z 5$ needs to be restored through other possible sources. In this case, the amount of required power to restore $Z 5$ is $700 K W$. The calculated $Q$-matrix for $F 3$ at this state $\left(s_{\text {peak }}\right)$, which is considered to be the peak load, is calculated using simulations results in peak condition.

$$
Q_{F A_{3}}\left(s_{k}, a_{i j}\right)=\begin{gathered}
F 4,2 \\
Z 1 \\
Z 1 \\
Z 3 \\
Z 5
\end{gathered}\left[\begin{array}{ccccc}
.98 & .61 & 0 & 0 & 0 \\
.65 & 1 & .06 & 0 & .01 \\
0 & .72 & .22 & .78 & .09 \\
0 & .97 & .85 & .98 & .77
\end{array}\right]
$$

Where $F i, j$ represents the alternative source feeder number $i$ connected to $F A_{3}$ through switch number $j$. The amount of available power from $F 6$ is $900 \mathrm{KW}$. Where .98 represents the degree of suggestion for $F 3$ to receive power from $F 6$ through tie switch number 6 to restore $Z 5$. Each row of the $Q$-Matrix represents the degree of suggestion for receiving power from possible feeder agents. Using the corresponding $Q$-Matrix for the current state, feeder agent 3 can find the optimal reconfiguration for restoration using equation 6.14. The accumulated reward for restoration through tie switch with $F 6$ is .98 for $Z 5$. Since the calculated index 
for receiving power from $F 6,6$ is the largest, $F 3$ starts negotiating with $F 6$ and after confirmation, tie switch number 6 is closed and the power is restored through feeder 6. Fig. 7.4 shows the WVSC one line diagram during the fault and after reconfiguration for cases I and II.

The $Q$-value for the recent transition should be updated using equation 6.12. The updated value for $Q_{44}$ with the learning rate of 0.01 is increased to 0.99, which shows an improvement in the confidence of taking the corresponding action. The updated Q-Matrix will be used for future decision makings and is shown below.

$$
Q_{F A_{3}}\left(s_{k}, a_{i j}\right)=\begin{gathered}
F 4,2 \\
Z 1 \\
Z 1 \\
Z 3 \\
Z 5
\end{gathered}\left[\begin{array}{ccccc}
.98 & .61 & 0 & 0 & 0 \\
.65 & 1 & .06 & 0 & .01 \\
0 & .72 & .22 & .78 & .09 \\
0 & .97 & .85 & .99 & .77
\end{array}\right]
$$

\subsubsection{Case II}

Line to line fault in zone 2 of feeder 4 . The fault location is shown with $(\star)$ in Fig. 7.4. The simulation conditions are the same as case I, except here it is a line to line fault. The $Q$-matrix for $F 4$ in the peak state is:

$$
\begin{aligned}
& \begin{array}{lllll}
F 8,15 & F 8,12 & F 3,2 & F 7,9 & F 3,8
\end{array} \\
& Q_{F A_{4}}\left(s_{k}, a_{i j}\right)=\begin{array}{c}
Z 1 \\
Z 14 \\
Z 11 \\
Z 10
\end{array}\left[\begin{array}{ccccc}
.47 & .61 & 0 & 0 & 0 \\
1 & .97 & .38 & 0 & .08 \\
.32 & .22 & .96 & .27 & .32 \\
.07 & .04 & .49 & 1 & .89
\end{array}\right]
\end{aligned}
$$

In this scenario, using equation $6.14, F 7$ is chosen to restore the power to zone 10. The updated values for $Q_{44}$ is 1 .

It has been demonstrated through the obtained results that the proposed MAS design can find a sub-optimal target configuration in every case, using either mathematical optimization or learning knowledge, if it exists. It should be noted that the learning solutions are more reliable than the mathematical programming approach, because they will be updated over time as the system runs and reflect real-world experiences. 


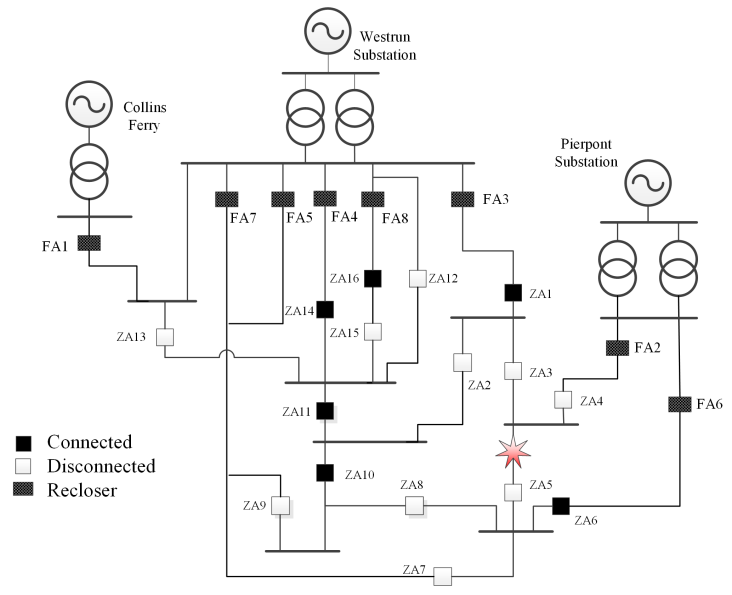

(A) Case I

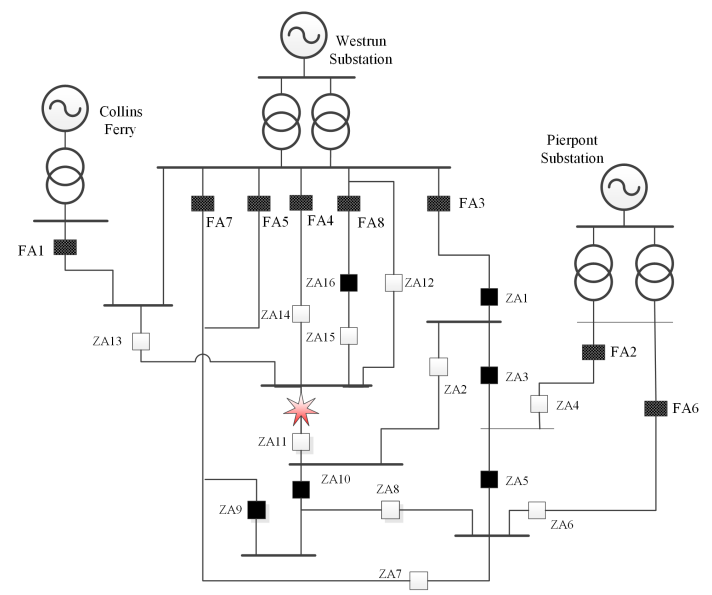

(B) Case II

Figure 7.4: Case I and Case II Reconfigurations

\subsection{Performance Analysis}

The restoration time is a significant index and the time consumed by agents to make restoration decisions matters. Using the proposed MAS framework with learning capability make the restoration faster than similar previous work. The intended MAS structure is for power distribution systems that are technically advanced and where all agents are equipped with communication capabilities, processing units and switches that can be operated automatically. These type of systems can be operated centrally from the control center or can be operated using decentralized, hybrid or hierarchical approaches. Proposed MAS architecture is different from centralized [21, 22], decentralized [24, 67], hybrid [23] approaches presented in previous work for using a hierarchical MAS architecture and learning based decision making.

Table 7.4 shows the number of required communication messages during the restoration for centralized, decentralized, hybrid and proposed approaches, assuming that the fault is located and all the zones are isolated. Fewer number of messages means less delay and computational burden for agents to manage the zones and can lead to a faster service restoration.

Table 7.4: Number of Communication Messages

\begin{tabular}{c|c|c|c|c}
\hline Number of Agents & Centralized & Decentralized & Hybrid & Proposed Method \\
\hline 21 & 20 & 38 & 42 & 16 \\
\hline 37 & 34 & 54 & 62 & 32 \\
\hline 123 & 108 & 76 & 82 & 58 \\
\hline
\end{tabular}


The advantage of the proposed MAS design over hybrid and decentralized approaches is that each agent just transmits its own messages and does not carry the neighbor agents messages. Although with the rise of system scale, number of messages in decentralized and hybrid approaches is less than the centralized approach, they still require more communication messages than the proposed approach.

It should be noted that the proposed MAS design requires less communication messages transmitted than other approaches because the communications are goal driven and learning knowledge helps the agents to prevent useless communication operations. 


\section{Chapter 8}

\section{Communication Performance Analysis}

In multi agents systems synchronized real time information is a key factor for reliable control of power distribution systems. Hence, it is critical to define the communication requirements and design the reliable, secure and cost-efficient communication system. MAS require a two way reliable communication with guaranteed quality of service i.e. low latency and sufficient bandwidth.

\subsection{Latency and Reliability Formulations}

In an engineering context, "inform" and "request" are two common types of messages in data transferring among the agents. After "inform" and sending each data packet, agents wait for acknowledgement. If no acknowledgment is received within a timeout period, the sender will resend the data. The process is repeated up to a predefined number of times depending to application. This method ensures that either messages are delivered to the destination with reliability 1 , or the sender will be alerted of the loss of communication with a specific receiver. Such reliable communication is needed because agents need the synchronized data of the neighbors to make the decisions. This reliability comes at the cost of higher delay (due to retransmission). 


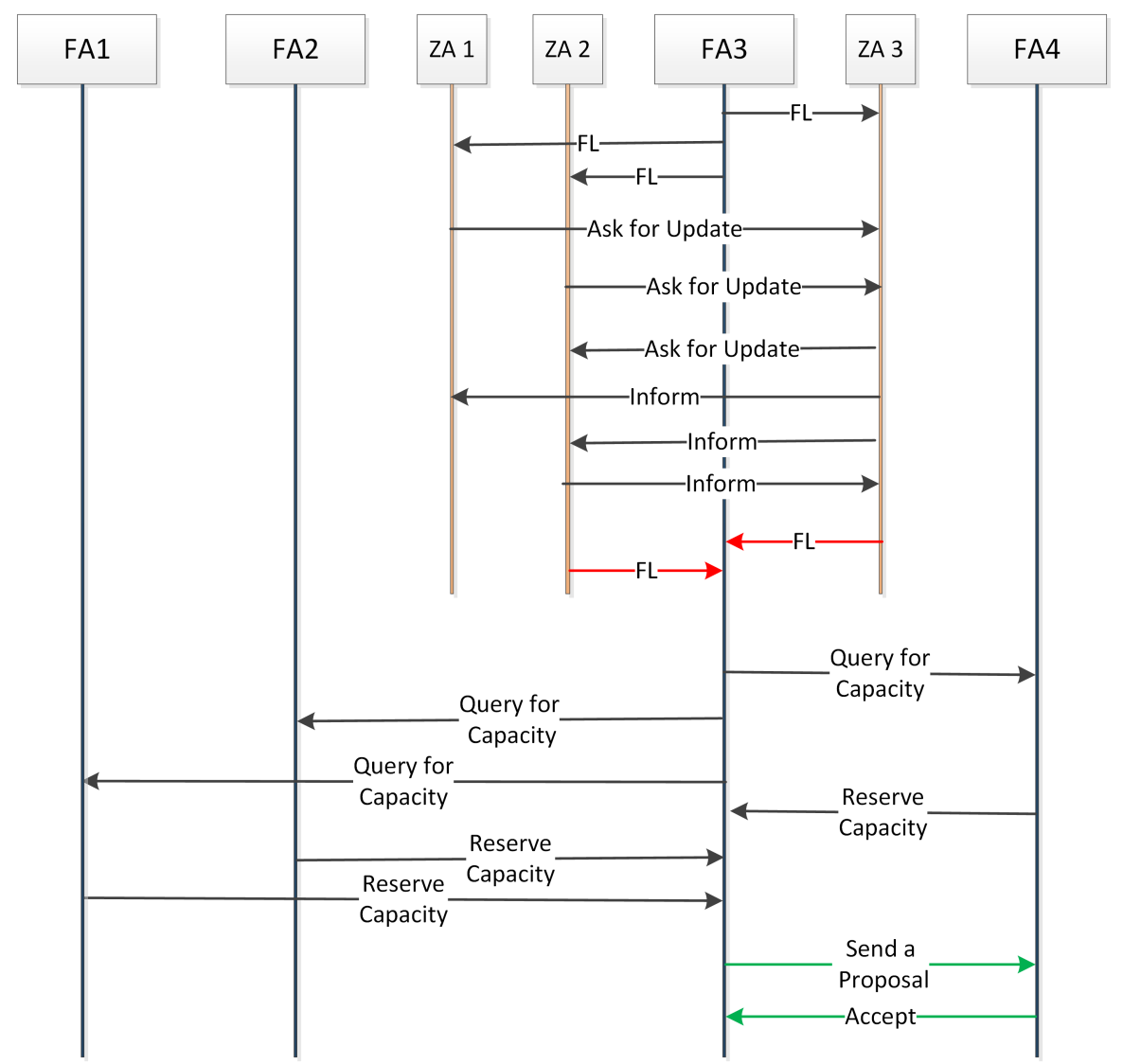

Figure 8.1: Communications Between Agents for FLiR

The communication messages vocabulary or "ontology" for messages depends on application. In the application considered in this work, information packet contains the voltage and current Phasor and the times tag for each phase i.e.

$$
\left(V, I, \psi_{v}, \psi_{i}, t\right)
$$

Where $V$ and $I$ represent the voltage, current and $\psi$ represent the phase angle for $\mathrm{V}$ and $\mathrm{I}$ at time t.

\subsubsection{Latency}

Real-time data communication in MAS includes online metering of data and reading messages within a very short time interval. Depending on the type of application, time requirements are different. In case of fault isolation, and from protection point of view, the fault current should be disconnected in approximately $100 \mathrm{~ms}$. The total signal latency may be represented as [68]: 


$$
\begin{gathered}
T=T_{\text {com }}+T_{c c} \\
T_{\text {com }}=T_{s}+T_{p}+T_{r} \\
T_{c c}=T_{\text {process }}+T_{b}
\end{gathered}
$$

Table 8.1: Time Delays for Latency Calculations

\begin{tabular}{|c|c|c|c|}
\hline$T_{\text {com }}$ & Communication delay & $T_{c c}$ & Processing delay \\
\hline$T_{s}$ & Serial delay & $T_{b}$ & Inter packet delay \\
\hline$T_{p}$ & Propagation delay & $T_{r}$ & Routing delay and media access \\
\hline
\end{tabular}

Propagation delay can be calculated by dividing the length of transmission media by the velocity of transmission media.

$$
\frac{L o M}{\nu}
$$

Where $L o M$ is the length of media and $\nu$ is the data transmission velocity. (e.g., $0.6 c$ to $c$, where $c$ is the speed of light). The routing and media access delay for a node can be calculated by adding all the routing and media access delays in between the node and the control center. Router and media access delay represent the latency introduced by the Network and MAC layer devices en route the message:

$$
T_{r}=\sum_{i=1}^{N o R}\left(T_{\text {rounter }}^{\text {th }}+T_{s}+T_{p}\right)
$$

Where NoR is the number of routers. Compared to $T_{r}, T_{s}$ and $T_{p}$ are negligible in the communication scenarios of interest $(<1 \mathrm{~ms})$. Therefore $T_{s}$ and $T_{p}$ are ignored in the rest of this work. For simplification we also assume that $T_{r}$ is same for all the links between sender-transmitter pairs. The value of $T_{r}$ is dependent on the network load in the shared communication medium (like wireless channels) or in 
the routing devices. The main factor in networks such as WiMAX is how much of the bandwidth is consumed by users and whether a part of the bandwidth is dedicated for MAS communication (using strict scheduling algorithms).

\subsubsection{Bandwidth}

The required bandwidth depends to the size of power system network and number of messages. Power system communication may be carried over a dedicated network, or a dedicated part of an existing network (sharing resources with existing traffic from other sources). In either case, as long as the amount of available bandwidth is sufficiently larger than the required BW (e.g., 20\% or more), when the amount of load becomes close to the available BW, the expected latency starts to grow quickly. Therefore, the effect of BW can be to a good extent accounted for by considering different levels of latency as long as the assumption of available BW being more than the offered load is correct.

\subsubsection{Reliablility}

Providing the system reliability has become one of the high priorities of utility companies. Power distribution system reliability is tied up to MAS communication system reliability and communication system malfunction can lead to huge problems in power system control and operation. However, wireless links are inherently unreliable. Reliability can be achieved through higher layer protocols and retransmission of data. Reliability calculations for communication system can be done based on the assumption that two corresponding agents can be in contact if and only if all the links and nodes in between work properly. Assuming that reliability can be described as the probability of a link or router successfully delivering a message, and assuming independence of links and routers, we can write:

$$
R_{\text {Agent }}=\coprod_{i=1}^{N o R} R_{i_{\text {rounter }}^{\text {th }}} \times \coprod_{i=1}^{N o L} R_{j_{\text {rounter }}^{\text {th }}}
$$

Where $R_{\text {Agent }}$ is the reliability of the reception for the considered agent and $N_{o} R$, $N o L$ represent number of routers and links correspondingly. $R_{i_{\text {rounter }}^{\text {th }}}$ and $R_{j_{\text {rounter }}^{\text {th }}}$ 
are the reliability of the routers and links in between two communicating agents. For simplification the reliability of routers and links are considered to be constant.

\subsection{Co-simulation of Power System and Commu- nication Network}

The simulation model has two parts. The first part is for simulating power system distribution network which is the WVSC by MATLAB Simpower Toolbox. The second part is the multi agent system which is implemented in Simulink by using user defined S-functions. The important issue of power/communication co-simulation is the accurately synchronization of two models in time. In this framework power system is modeled as continues time system which changes the system state variables with respect to time. However the communication network simulation model works using a discrete method. Matlab engine calls the output and derivative portions of the S-functions (agents) at minor time steps to compute the states for agents. More detail about the power/communication models is presented in the following. The multi agent system is implemented using the S-function blocks of MATLAB Simulink. Each agent is modeled by an S-function. An S-function is a computer language description of a Simulink block written in MATLABß. S-functions use a special calling syntax called the S-function API that enables you to interact with the Simulink engine. They follow a general form and can accommodate continuous, discrete, and hybrid systems. Since the MAS is mainly including agents communication, and communications are intrinsically discrete in time, MAS works in discrete mode and it also provide the base to model communication delays and latency. The measured data resolution is 16 samples per cycle, and agents can access to power system model simulation data with 16 samples per cycle resolution, the MAS discrete step simulation time is fixed on 0.001 second.

We assumed that the message reception rate in this specific application should be more than 0.999 percent. Based on this assumption the expected time delay for each technology is described in Table 8.2.

If the reception probability can be calculated by:

$$
P_{\text {reception }}=1-(1-P)^{N}
$$




$$
P_{\text {reception }}>.999
$$

Where $P$ is the probability of successful communication for each trial, $N$ is the number of send attempts for each message and $T_{L}$ is the required communication time for each link.

Table 8.2: Expected Delay for Each Communication Link

\begin{tabular}{|c|c|c|}
\hline Technology & $P$ & Expected delay for each link \\
\hline Wired & .999 & $2 T_{L}$ \\
\hline Wireless & .8 & $5 T_{L}$ \\
\hline Wireless & .9 & $3 T_{L}$ \\
\hline Wireless & .99 & $2 T_{L}$ \\
\hline
\end{tabular}

Table 8.3 shows the processing time and communication delay for each considered scenario. The total delay is calculated by summation of communication and processing delays range in different simulation trials. The processing time in the simulation is assumed related to the number of messages processed by each agent.

Table 8.3: Best and Worst Case Latency for MaS Structures with Wired and Wireless Communication Media (MS)

\begin{tabular}{|c|c|c|c|c|c|c|c|c|}
\hline \multirow{2}{*}{ MAS Architecture } & \multirow{2}{*}{ Number of Messages } & \multicolumn{3}{|c|}{$T_{\text {com }}$} & \multirow{2}{*}{$T_{c c}$} & \multicolumn{3}{|c|}{ Total Delay } \\
\hline & & Wired & Wireless $(.8)$ & Wireless $(.9)$ & & Wired & Wireless $(.8)$ & Wireless (.99) \\
\hline Centralize & 48 & $28-3323$ & $78-7500$ & $45-4527$ & 16 & 44-3339 & $94-7516$ & $61-4588$ \\
\hline Hierarchical & 37 & $49-5000$ & $125-12500$ & $75-7500$ & 4 & $57-5008$ & $133-12508$ & $83-7508$ \\
\hline Distributed & 92 & $40-4154$ & $102-10457$ & $69-6006$ & 3 & $46-4160$ & $108-10463$ & $75-6012$ \\
\hline
\end{tabular}

Length of communication media has a direct relationship with the latency and reliability of communication system. Length of Communication media in centralized structure is longer than the hierarchical and in hierarchical it's longer than the distributed one. Therefore the distributed architecture could be the optimal structure from reliability point of view.

In a centralized architecture, all the messages will be transmitted to control center in parallel. After the processing the data, control center sends the command signals 
to the corresponding agents. In a hierarchical structure, messages are transmitted in parallel in each hierarchy, but between the hierarchies, messages are transmitted in series (Fig. 8.2). In the distributed architecture there is no hierarchy and each agent's communication are done in parallel, therefore when the number of agents rise, number of transmitted messages per agent does not rise as much.

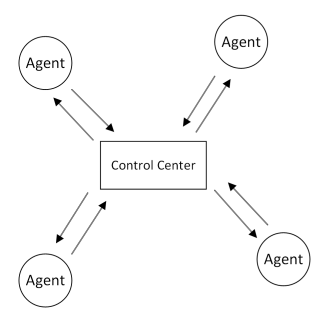

(A) Centralized

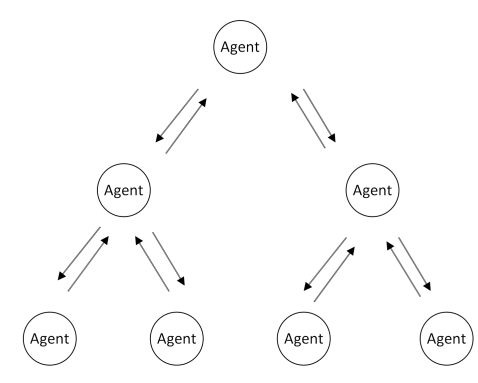

(B) Hierarchical

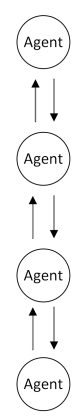

(C) Distributed

Figure 8.2: SERIES AND PARALlel COMmunicAtions in EACH ARCHiteCture

The investigated MAS in this work just contain 16 agents. That is why the centralized approach has the least latency. In case of larger networks the efficiency of distributed MAS structure won't be affected much while the other structures will be affected. Because in distributed architecture agents just talk to their neighbors, but in other architectures number of messages and consequently processing time will increase as the network size increase. In the simulated WVSC both centralized and distributed approaches have close latencies and could be the optimal choice.

In a sample simulation scenario a single phase to ground fault is created in the WVSC model and the MAS structure is considered to be hierarchical. Fig. 8.3 shows the fault voltage and current data before and after communication for this scenario, the first part shows the simulated power system waveforms, the second shows the corresponding agent sampled data and the third one shows the received signal by the neighbor agent through communication. 

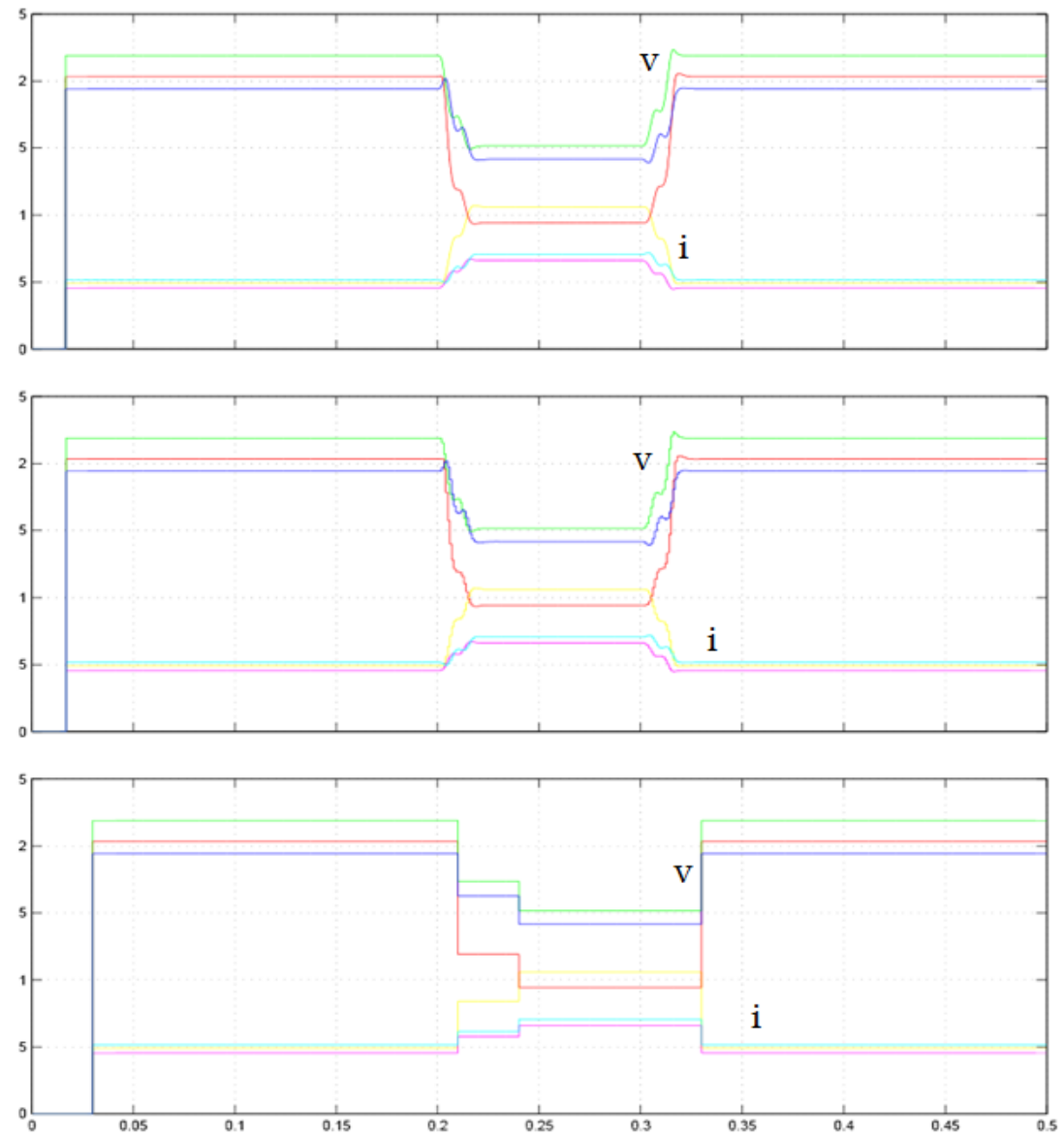

Time (ms)

Figure 8.3: Measurement Data $(V \& I)$ and Transmitted Data to Control Center for DisTRIBUted ARCHITECTURE OF MAS 


\section{Chapter 9}

\section{Load Shedding}

Power outages in distribution systems can create power mismatch between generation and loads. Power balance in an electricity grid must be maintained on a second by second basis since electricity cannot be stored easily in a cost effective manner. When a fault occurs, firstly the fault should be located to be able to isolate the faulted zone. Secondly, the service should be restored to the loads which are in the fault-free areas.

The objective of this work is to develop a distributed Multi Agent System (MAS) to restore the power to high priority loads after the outages happen. After fault location and isolation using the available techniques [12, 13], MAS tries to find the optimal reconfiguration to re-energize the fault-free zones. In general there are three ways to restore the service for fault free zones,

1. Direct feeder restoration: the zones upstream the fault zone can be restored by reclosing the recloser at the top of each feeder.

2. Load transfer restoration: If other feeders have enough extra power to provide for out-of-service areas, and there are tie switches that can transfer the power.

3. Load-shedding restoration: load shedding restoration is when there is not enough available power to restore all the out-of-service zones. If there are Distributed Generation (DG) sources in the out of service zones, they can be used to restore some loads. If the sum of DG capacity and available power from other feeders is less than the zone's power demand, load shedding can be done to restore the service to as many loads as possible. 
It is difficult to determine the amount of the load to be shed when the system loads change dynamically and has significant penetration of generations that are stochastic in nature, such as wind turbines, solar cells, etc. In this chapter, authors presented a distributed MAS-based system that finds the near-optimal configuration for restoration using the available local data. Proposed MAS agents in the bottom-up order are Load Agent (LA), Distributed Generation Agent (DGA), Zone Agent (ZA), Feeder Agent (FA) and Substation Agent (SA). A new fuzzybased load prediction methodology has been used in this work that allows agents (LA and DGA) to predict their future load profile and update their knowledge with the online monitoring data. In proposed framework, FAs are responsible for restoration and load shedding decision makings and use a nonlinear programming technique to obtain the optimal reconfiguration for restoration.

\subsection{Multi-Agent System}

This section introduces the proposed MAS architecture, the agents responsibilities and function design for load shedding and restoration application in PDS.

\subsubsection{Agent Architecture}

The proposed MAS comprises five types of agents shown in Fig. 9.1. Their responsibilities are defined below,

- Load Agent (LA):

A LA resides at any bus that has connected load. LA monitors load parameters, such as voltage, current, and active power consumption. Due to the fact that loads have different priorities, LA will need to know its own priority as well. During an outage, if there is not enough power available to serve all loads, ZA's will send a control signal to lower priority loads to disconnect.

- Distributed Generation Agent (DGA):

A DGA monitors the DG parameters, such as DG voltage and status. DGA provides its information to the corresponding ZA, including maximum capacity of DG and power generated by DG. During an outage ZA will negotiate 
with its DGAs to see if there is enough power available with DGs to supply the requested loads.

- Zone Agent (ZA):

Zone Agent act as a coordinator between LAs, DGAs and FAs, it informs the FA about the amount of the power it requires and its loads priority and communicates the restoration decisions to its LAs and DGAs. ZA is also responsible for load prediction, detecting and isolating the fault zone when there is a fault in its zone.

- Feeder Agent (FA):

Makes the reconfiguration, restoration and load shedding decisions based on the information it gathered from its ZAs and also the neighbor FAs. After decision making, FA sends the decisions to ZAs and they perform the required switchings with respect to FA commands.

- Substation Agent (SA):

$\mathrm{SA}$ is responsible for negotiations with other SAs when there are tie switches with other SAs and also for SCADA level communications.

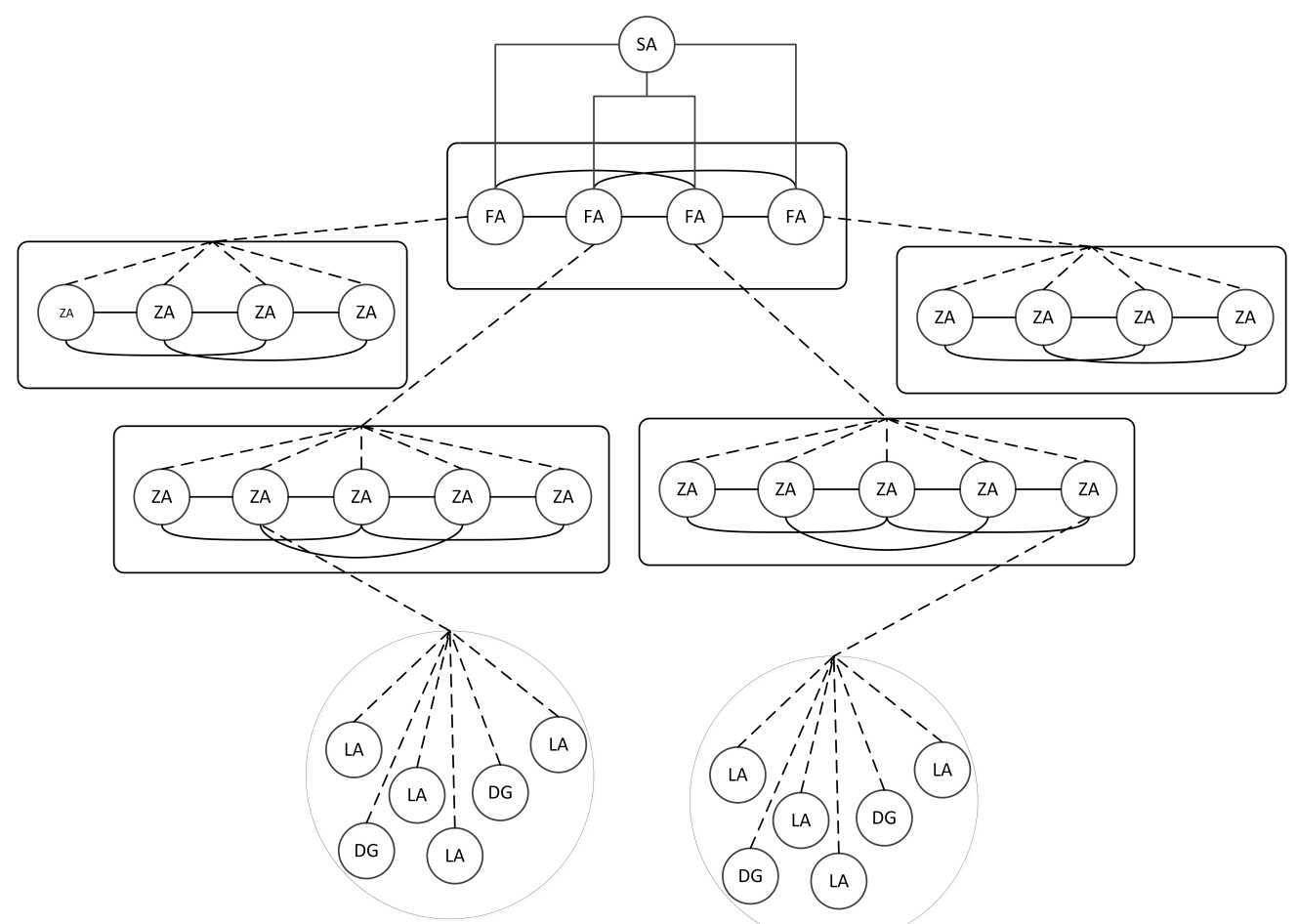

Figure 9.1: MAS ARChitecture 


\subsubsection{Agent Application Design}

Each type of agent processes different type of data. The knowledge for each type of agent is summarized in Table 9.1. The term fact is used to represent the agent's knowledge. Facts contain information either of the associated agent itself or the agent external environment.

Table 9.1: Agent's Facts

\begin{tabular}{|c|l|c|}
\hline Agent & Fact & Value \\
\hline \multirow{4}{*}{ LA } & Priority & Vital/Semi-vital/Non-vital \\
\cline { 2 - 3 } & Bus Voltage & p.u \\
\cline { 2 - 3 } & Load status & on/off \\
\cline { 2 - 3 } & Required Power & MW \\
\cline { 2 - 3 } & Load Priority & integer \\
\hline \multirow{4}{*}{ DGA } & Bus Voltage & p.u \\
\cline { 2 - 3 } & DG status & on/off \\
\cline { 2 - 3 } & Capacity & MW \\
\cline { 2 - 3 } & type & Solar/Wind/... \\
\hline \multirow{5}{*}{ ZA } & Bus Voltage & p.u \\
\cline { 2 - 3 } & Bus Current & A \\
\cline { 2 - 3 } & Top Breaker status & on/off \\
\cline { 2 - 3 } & End Breaker status & on/off \\
\cline { 2 - 3 } & Power Consumption & MW \\
\cline { 2 - 3 } & Predicted Demand & MW \\
\cline { 2 - 3 } & Connected LAs & ID \\
\cline { 2 - 3 } & Connected DGs & ID \\
\cline { 2 - 3 } & Fault Zone & 1/0 \\
\hline \multirow{5}{*}{ FA } & Feeder Voltage & p.u \\
\cline { 2 - 3 } & Feeder Current & A \\
\cline { 2 - 3 } & Recloser Status & on/off \\
\cline { 2 - 3 } & Neighbor FAs & ID \\
\cline { 2 - 3 } & Connected ZAs & ID \\
\hline
\end{tabular}

\subsection{Active Learning Method}

Active Learning Method (ALM) is a universal fuzzy modeling method. This method has two main features that make it very similar to the way that human mind processes different phenomena. ALM mainly deals with phenomena 
modeled as Multi Input Single Output (MISO) systems and assumes that the proposed MISO systems comprise several Single Input Single output (SISO) systems, so ALM only deals with one input and one output at a time. This feature makes ALM very similar to the human brain. In addition, ALM uses a novel fuzzy interpolation method called Ink Drop Spread (IDS) [69] to construct the individual SISO systems. To model and estimate a MISO system, ALM decomposes it into many SISO systems. Then as shown in Fig. 9.2, each SISO system is expressed in a data plane created by gathering data from system when it is fed by different inputs. Each data plane provides two distinct features of the given data; the first feature is a curve called narrow path that is extracted from IDS method which will be discussed in the next section. The proposed narrow path indicates output behavior regarding to only one input; In other words, each narrow path determines the best function describing the relation between the output and one specific input. The other feature is called the spread of the points around the narrow path. Spread demonstrates the deviation of the data points from the narrow path. In other words, spread determines the degree of the dependency of the output on each specific input. As discussed later, this degree can be used to obtain the firing rate of each fuzzy rule $[69,70]$.

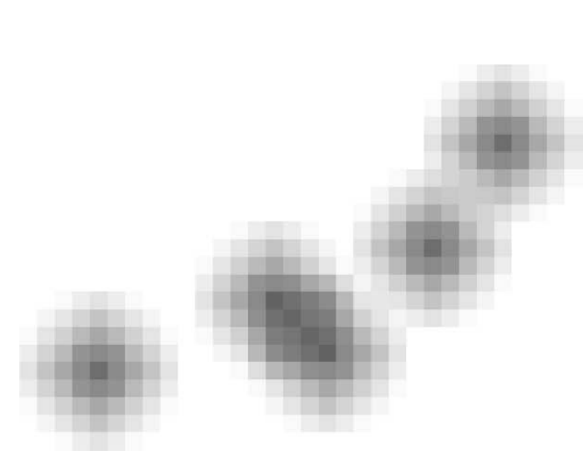

(a)

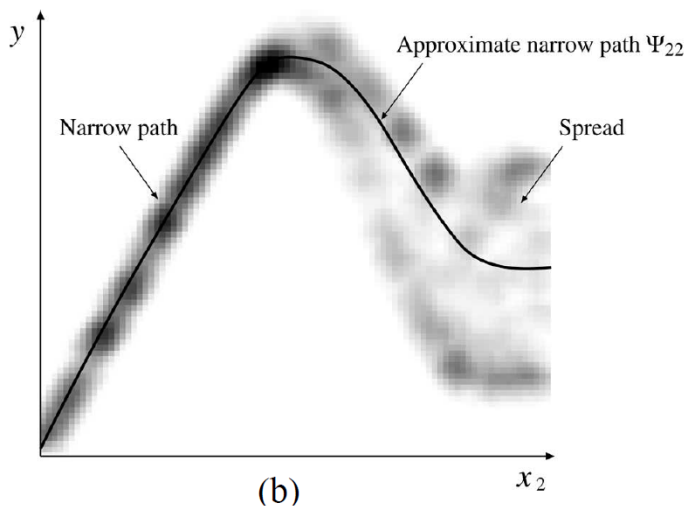

Figure 9.2: IDS plane: (A) Five Data points Spread on a Plane (B) InK Spreads and Narrow PATH

\subsubsection{Ink Drop Spread (IDS)}

IDS is the core part of ALM which extracts narrow paths and spreads [71, 72]. In IDS we assume that every point in the input-output plane acts as a light source. The illuminating pattern of the light source has conical shape and is considered as a spatial fuzzy membership function. The illuminating patterns of different 
points are superposed forming new bright areas [71]. This concept is illustrated in Fig. 9.3. Finally, the narrow path is extracted by applying the center of gravity defuzzification method. After the extraction of narrow path, the spread value for every point in the narrow path is obtained by measuring the deviation of data points around the narrow path.
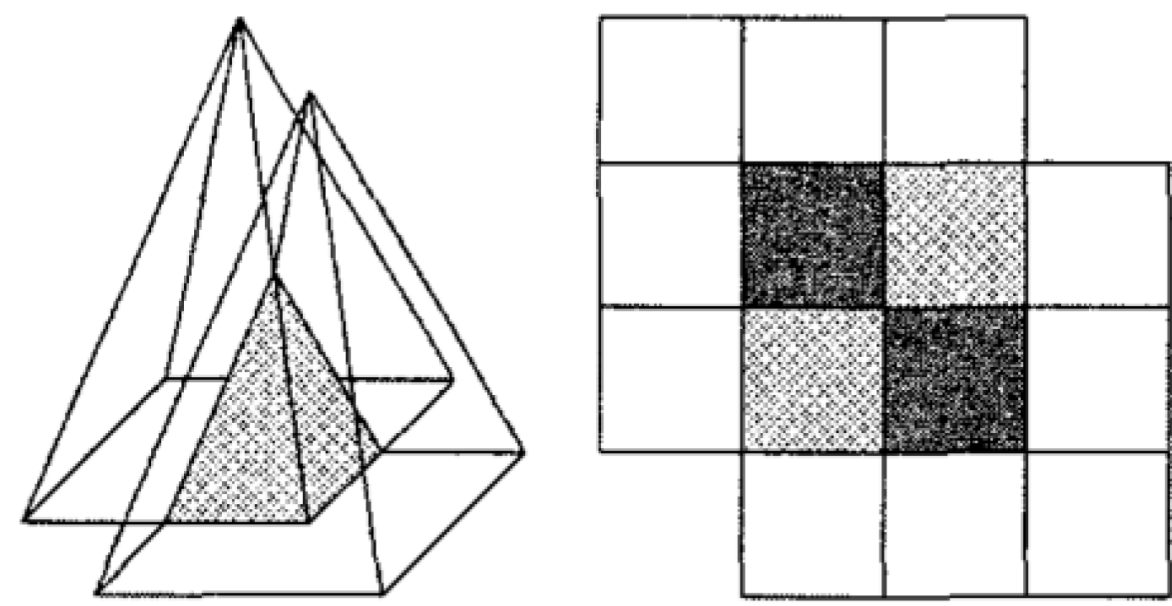

Figure 9.3: EACH DATA POINT HAS A PYRAMIDAL FUZZY MEMBERShIP

\subsubsection{Combination of Fuzzy Rules}

According to Shouraki et al. [73] ALM fuzzy rules have a general form shown below and the fuzzy scaling factors for the rules will be obtained by using the difference between the membership degree of the points on the narrow path and the membership degree of their neighbor points on the plane:

$$
\begin{aligned}
& \text { If }: x_{1} \text { is } A_{1 i}, \cdots \text { AND } x_{n} \text { is } A_{n m} \\
& \text { Then : y is } \beta_{1 i} U_{1 i}, \cdots \text { OR } \beta_{n m} U_{n m}
\end{aligned}
$$

Where,

$A_{n m}:$ is the $m^{\text {th }}$ domain of $n^{\text {th }}$ input

$U_{n m}$ : is the narrow path related to $n^{\text {th }}$ input in its $m^{\text {th }}$ domain

$\beta_{n m}$ : is the fuzzy number representing the deviation of the data points from the narrow path 
The IDS method may be used as a processing engine for the combination rule which can also be represented mathematically as follows:

$$
\begin{aligned}
& \text { If }: x_{1} \text { is } A_{1 i}, \cdots A N D x_{n} \text { is } A_{n m} \\
& \text { Then : } y \text { is } \frac{\frac{1}{\alpha_{1 i}} U_{1 i}}{\frac{1}{\alpha_{1 i}}+\cdots+\frac{1}{\alpha_{n m}}}, \cdots, O R \frac{\frac{1}{\alpha_{n m}} U_{n m}}{\frac{1}{\alpha_{1 i}}+\cdots+\frac{1}{\alpha_{n m}}}
\end{aligned}
$$

Where $\alpha_{n m}$ is the average of the spread function in $m^{\text {th }}$ domain of $n^{\text {th }}$ input calculated by center of area method.

\subsection{ALM-Based Load Prediction}

In the recent times, machine learning methods have proved to be reliable nonlinear function approximators [74]. Climate variables and power consumption of a load has been used for the prediction. Table 9.2 shows the list of variables used for the models evaluation.

\section{TABLE 9.2: VARIABLES USED IN LOAD PREDICTION MODELS}

\begin{tabular}{|l|c|}
\hline Variable & Description \\
\hline Day & Day of the month \\
\hline Month & Month \\
\hline Time & Time of day \\
\hline OT & Outside temperature $\left(C^{\circ}\right)$ \\
\hline RH & Relative humidity $(\%)$ \\
\hline SR & Solar radiation $\left(W / m^{2}\right)$ \\
\hline WD & Working day \\
\hline AP & Active power $(k W / h)$ \\
\hline
\end{tabular}

ALM splits a MISO system into several SISO systems as shown in Fig. 9.4. There is a SISO system for each of the variables mentioned in Table 9.2. The key idea here is to observe the output behavior of a MISO system with respect to each input variable, which limits the variation range of other effective inputs. Therefore, SISO systems model the MISO system output in a specific partition of 

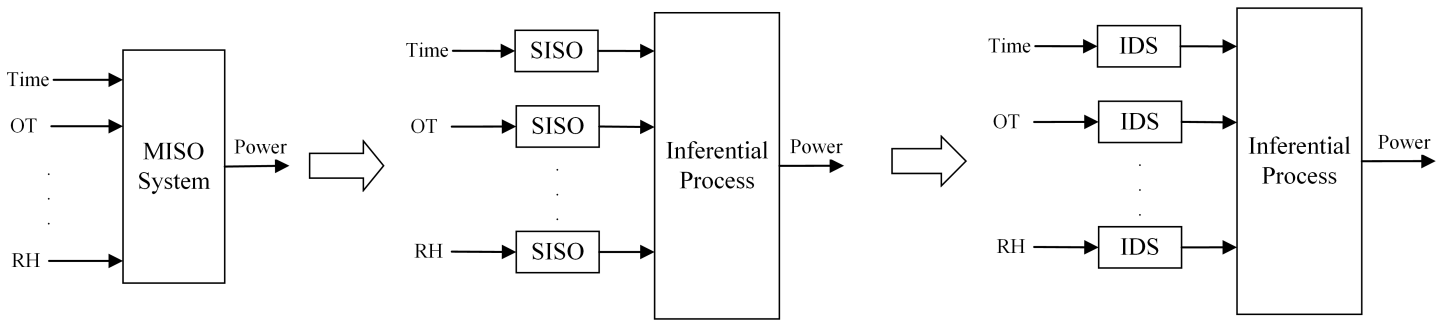

Figure 9.4: ALM splits a MISO System into SEVERAL SISO Systems, Each SISO System IS MODELED BY AN IDS PLANE

the feature space with respect to the unpartitioned input variables. Each SISO system is then modeled by an IDS unit.

In order to extract the two important features i.e. narrow path and fuzzy spread interpolation operator, IDS method is used. Fig. 9.5 shows the IDS plane for the annual daily power consumption vs. season of the year for a residential zone.

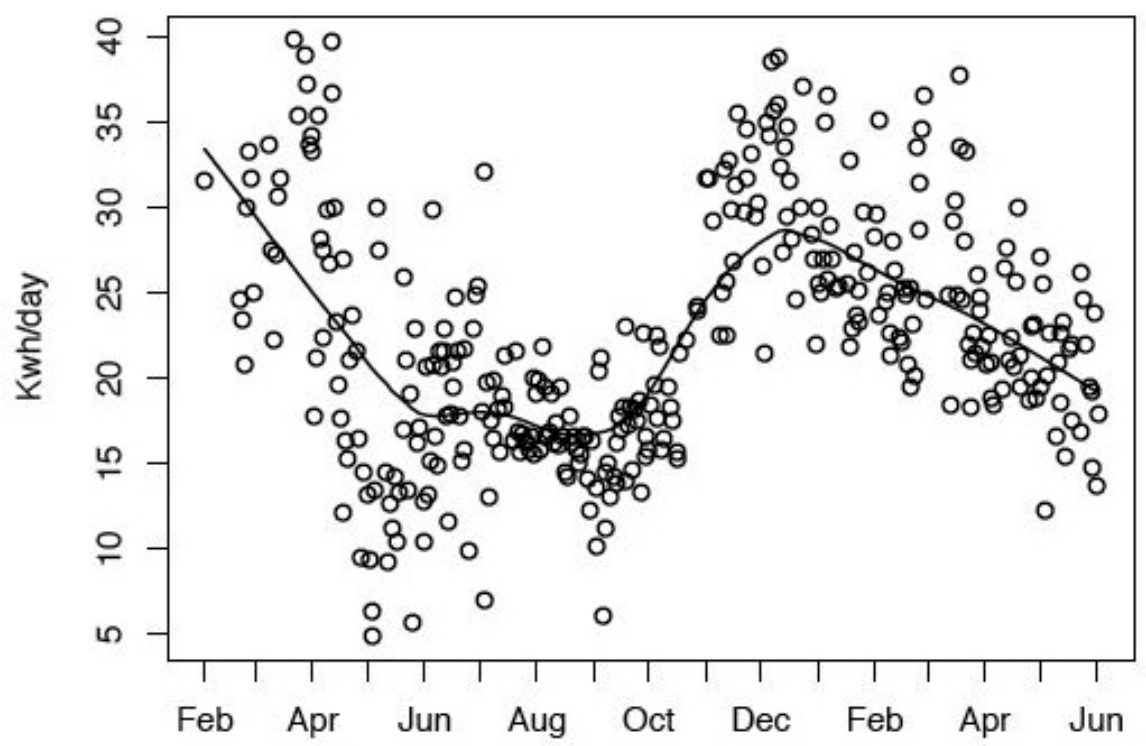

FiguRE 9.5: IDS PLANE FOR SEASONAL EFFECT ON POWER CONSUMPTION [3]

When the data for a specific month are more spread around the narrow path, it means that the confidence level of obtained knowledge is less than when the data are closer to the trajectory. This is similar to the notion of fuzzy membership functions. The spread functions, which show the amount of spread of data on each plane resulting from the effects of other variables, can be calculated using a method presented in [75] by Shouraki and Honda. Then the output of the system can be calculated by: 


$$
P \operatorname{Demand}(\text { day, } \text { month }, \cdots)=\frac{\frac{1}{\alpha_{d}} U_{d}+\frac{1}{\alpha_{m}} U_{m}+\cdots}{\frac{1}{\alpha_{d}}+\frac{1}{\alpha_{m}}+\cdots}
$$

Where $\alpha$ represent the spread value for each SISO system output $(U)$. Here $U_{m}$ represents the output of SISO system for the month feature and $\alpha_{m}$ represent the spread function value.

\subsection{Load Shedding Optimization}

In this section the service restoration process is described when there are DG's available in the network.

\subsubsection{LS algorithm flow}

When there is a fault in a PDS feeder, after the fault location and isolation process, the fault upstream zones can be restored by re-closing the recloser. Restoration for fault downstream zones is more challenging. The downstream restoration zone refers to the area between downstream fault zone to the normal open tie switches to neighboring feeders. There are two possible sources to restore the downstream zones, available power from DGs and transferable power from neighboring feeders. If the sum of the available power from DGs and supporting feeders could absorb all incoming loads, no load shedding will be done, and the restoration optimization process determines to what feeders loads should be transferred. Otherwise, lower priority loads should be shed to avoid overloading the supporting feeders and DGs, while maximizing the service recovery. Fig. 9.6 shows the flowchart for the service restoration process.

\subsubsection{LS Formulations}

When fault isolation process is completed, FA starts communicating with neighboring FAs to restore the service to zones downstream the fault location. Considering various operating conditions of PDS, load shedding should be treated as a dynamic problem. Since the loads connect to, or disconnect from the power system continuously, it is necessary to dynamically update the information on load 


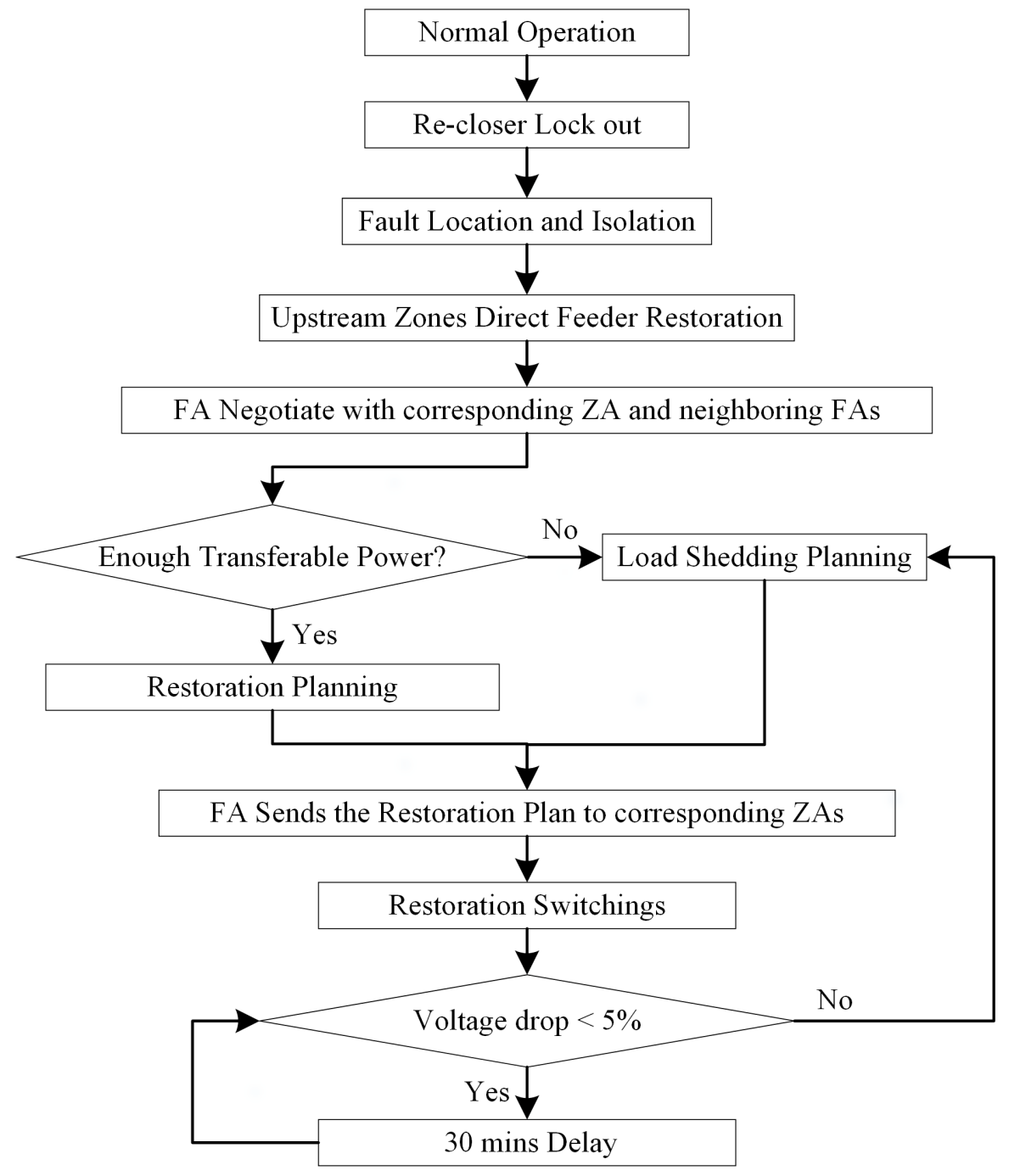

Figure 9.6: Restoration Flowchart

connection so as to avoid insufficient or excessive load shedding. Therefore, FAs update their knowledge about the existing loads in their zones periodically (every 5 mins). Through communication with its ZAs, FA is aware of its zones demand and also available power from DGs. Total net demand active power $P_{\text {net }}$ for a zone can be calculated using:

$$
P_{n e t}=\sum_{i=1}^{m} P_{D G_{i}}-\sum_{j=1}^{n} P_{L_{j}}
$$

Where $P_{D G_{i}}$ is the active power generation from $i^{t h}$ DGA and $P_{L_{j}}$ is the $j^{\text {th }}$ LA's load. Considering the available power, requested demand and PDS network constraints, FA will tries to find the optimal configuration for each phase $(a, b, c)$ using nonlinear programming. 


$$
\begin{aligned}
& \text { Objective function: } \quad \operatorname{Max}\left\{\sum_{i=1}^{n} J(s)\right\} \\
& J(s)=\gamma_{i_{v}}\left(\rho_{v} \cdot P_{i_{v}}\right)+\gamma_{i_{s v}}\left(\rho_{s v} \cdot P_{i_{s v}}\right)+\gamma_{i_{n v}}\left(\rho_{n v} \cdot P_{i_{n v}}\right)
\end{aligned}
$$

Where $\rho_{v}, \rho_{s v}, \rho_{n v}$ are the priority values for vital, semi-vital and non-vital loads in the $i^{\text {th }}$ zone and $\gamma_{i_{v}}, \gamma_{i_{s v}}, \gamma_{i_{n v}}$ are the binary values determining whether the $i^{\text {th }}$ zone vital, semi-vital and non-vital loads should be restored or not (1: restored; 0: not restored). $P_{i_{v}}, P_{i_{s v}}$ and $P_{i_{n v}}$ represent the maximum net predicted vital, semi-vital and non-vital power demands for the next 4 hours window for $i^{\text {th }}$ zone loads using the method described above. The Maximum predicted value is used to prevent short time service restorations ( $\preceq 4$ hours) for out of service loads. The fulfillment of the objective should be obtained satisfying the following constraints.

\section{Constraints:}

1. Loads Priority: Semi-vital loads can be restored in case that all the vital loads are restored and respectively non-vital loads can be restored only if all the semi-vital loads are restored,

$$
\gamma_{i_{v}} \succeq \gamma_{i_{s v}} \succeq \gamma_{i_{n v}}
$$

2. Zone Constraint: The sum of power flows into any zone should be equal to the sum of power flows leaving the zone, plus the zone's power demand.

$$
\sum_{i \in \text { in }_{i}} P_{i-f}=\sum_{i \in \text { out }_{i}} P_{i-f}+P_{i f}
$$

where $\mathrm{f}$ is the phase $(a, b$ and $c)$. $i n_{i}$ and $o u t_{i}$ are the set of input and output power flows into and out of zone $i$. $P_{i f}$ represents the net power consumption by zone $i$ itself. 
3. Source Capacity Constraint: The sum of the power flows going out of a zone should not exceed the total available capacity of the respective zone.

$$
\sum_{i \in \text { out }_{i}} P_{i-f} \leq P_{\text {cap-if }}
$$

where $P_{c a p-i f}$ is the available capacity in phase $f$ of $i^{\text {th }}$ zone.

4. Radial configuration Constraint: The radiality constraint ensures that only one branch feeds each zone.

$$
\sum_{i \in i n_{i}} S_{i-f} \leq 1
$$

where $S$ is the status of switches at the feeding side of the zone and is defined as follows: $S_{i}=1$ if switch $i$ is closed and $S_{i}=0$, otherwise.

5. Voltage Drop Constraint: Voltages at end of zones should be within standard limits. The voltage drop is calculated by the $K-d r o p$ value

$$
K_{\text {drop }}=\frac{\text { Percent_Voltage_Drop }}{k V A \_ \text {mile }}
$$

$K$ - factor is the voltage drop percent down a line that is one mile long and serving a balanced three-phase load of $1 k V A$. $l$ is the one way distance and $k V A$ is the actual load of the line [61].

The voltage drop along a line segment is computed by:

$$
V_{\text {drop }}=K_{\text {drop }} \cdot k V A \cdot \text { mile }
$$

Voltage drop should be within the limits i.e. $V_{d} \leq 5 \%$. These constraints will ensure that while optimizing the restoration, the voltage constraints are also satisfied.

\subsection{Case Studies}

In order to study the performance of proposed schemes, introduced in previous sections, a real power system, West Virginia Super Circuit (WVSC), is simulated. 
The simulation model has two parts. The first part is the simulation of the West Virginia Super Circuit (WVSC) in MATLAB Simpower Toolbox [65]. The second part is the MAS which is implemented in Simulink by using user defined S-functions. Since both MAS and power system model are implemented in MATLAB Simulink there is no need for an interface between the two models. This means MAS and power system model can work in real time, which provides a simpler and more accurate simulation model.

Two case studies results are presented in this section. In each scenario, after the fault occurrence and recloser operation all the feeder zones are deprived of power. It is assumed that the fault has been located and isolated by the fault location and isolation algorithm and the fault upstream zones are restored by reclosing the recloser.

\subsubsection{Test System}

There are 16 switches installed in two feeders of West Virginia Super Circuit (WVSC) to enable the system (Fig. 9.7). Switches are Cooper DAS-15 type three-phase vacuum switches with $15 \mathrm{KV}, 630 \mathrm{~A}$ ratings. 7 switches are normally closed and the other 9 switches will be used for reconfiguration and restoration applications. Transformers in each feeder are 138/12.5 KV and 33.6 MVA.

\subsubsection{Case I: Load Shedding Restoration}

A single phase to ground fault is applied to zone 3 (ZA3) of feeder 3. To restore downstream zones, FA3 will start negotiating with the neighbor feeder agents which are FA6, FA4 and FA7. F3 knows the predicted required power to restore zone 5 and asks for the required power for restoration. Both FA6 and FA7 respond with the capability of transferring the required power to feeder 3. By solving the optimization problem using the proposals and circuit's data, FA6 proposal was decided to be accepted without need for any load shedding. Fig. 9.8 shows the voltage profile from the ZA1, ZA3 and ZA5 point of view, when ZA5 gets the power from FA6. The green and blue colors represent the voltage profile for ZA1 and ZA3, red line shows the voltage profile for ZA5. Since the ZA1 and ZA3 are restored by direct feeder restoration, they have a quicker restoration than ZA5. 


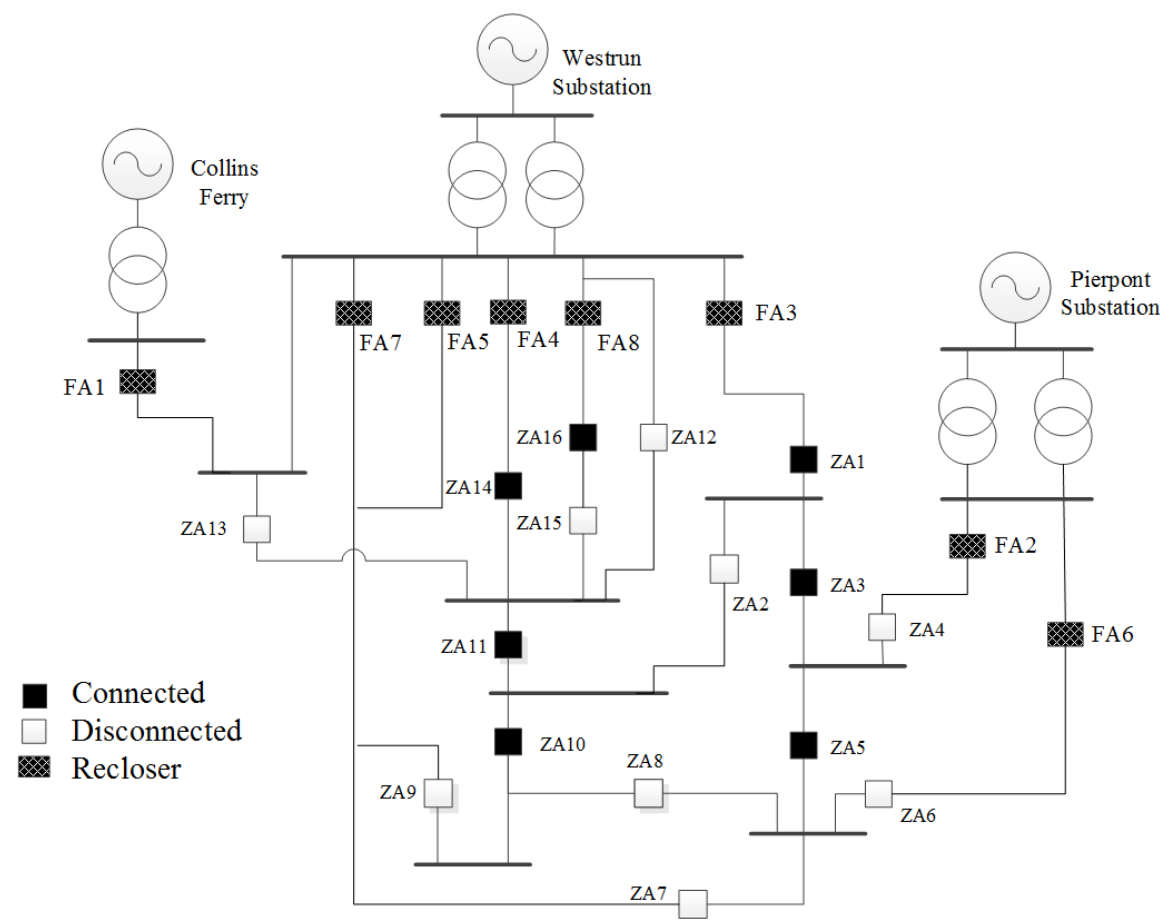

Figure 9.7: West Virginia Super Circuit

ZA5 is restored after 3.5 seconds and at $4^{\text {th }}$ second, ZA5 sheds more loads to improve the voltage profile.

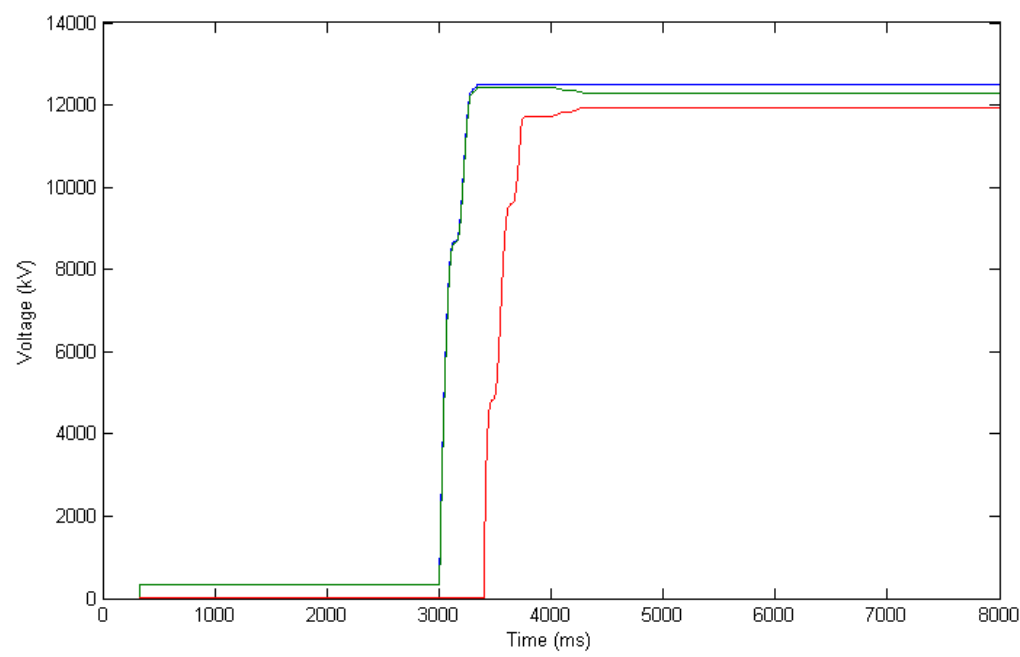

Figure 9.8: Voltage Simulation Result for Case I

\subsubsection{Case II: Including DG's}

In this case a similar scenario like case I is studied, but there are DGs installed in Zone 1 and 5 of feeder 3. After the fault location and isolation, FA3 negotiations 
with neighboring feeders determine despite the availability of power from DG's, there is not enough power available from neighboring feeders to restore the out of service loads through tie switches. Therefore, FA3 decides to run the loads shedding program and shed some of the Non-vital loads before initiating the restoration switching. Fig. 9.9 shows the voltage profile from the ZA1, ZA3 and ZA5 point of view. DG's are connected to network after 5 seconds, and improve the voltage profile.

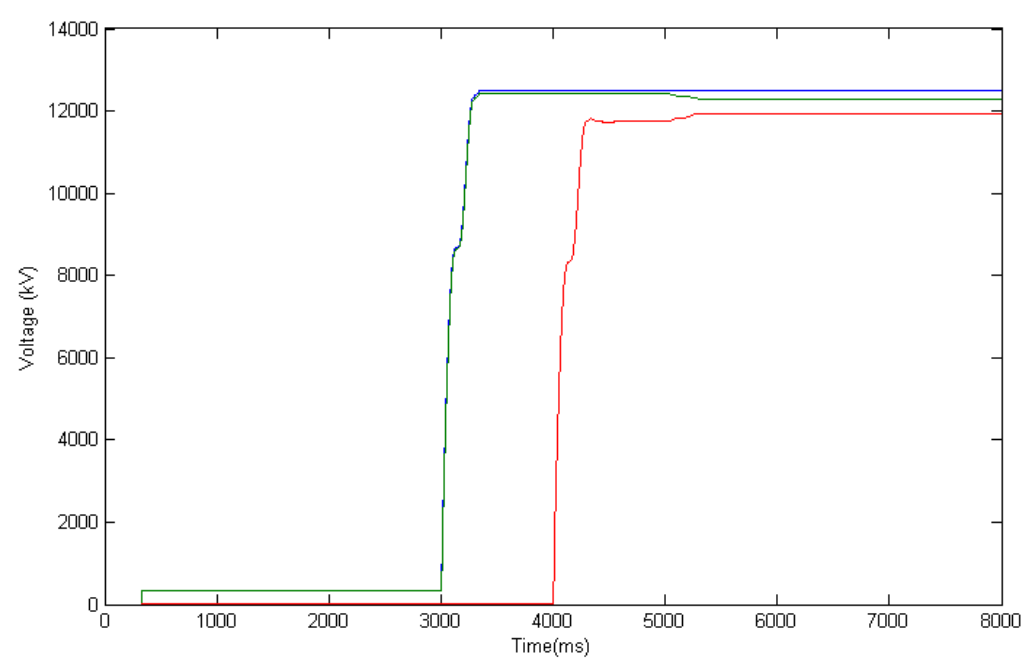

Figure 9.9: Voltage Simulation Result for Case II 


\section{Chapter 10}

\section{Hardware Implementation}

Fault location approaches can be deployed by utilities to estimate the location of electrical faults in a Power Distribution System (PDS) so as to expedite service restoration and improve power quality. For fault location applications, utilities in the past have relied primarily on customer phone calls and supervisory control and data acquisition (SCADA) data to find out what was going on with their systems. Rarely could the utility automatically and accurately determine outage extent and location.

A key driver and measurement of utility effectiveness is the reliability of provided power to its customers. When permanent faults occur on overhead lines, having automated feeders can greatly enhance distribution grid reliability and reduce restoration of fault-free zones from several hours to a few seconds or minutes. More-accurate locating of fault zone will shorten the fault investigation (patrol) time, which in turn can reduce the total restoration time and duration of the outage experienced by the customers [76, 77].

Considering the existence of multiple laterals and sub laterals tapped off the main feeder in different locations of PDS, time varying unbalanced load profiles, power injection at different location of system by distributed generation, fault locating in PDS is a challenging task. Existing Fault Location and Isolation (FLI) algorithms could be grouped in three main categories;

1. Impedance based methods; these methods usually calculate the apparent impedance sequences using measurement points data and estimate the possible fault locations based on iterative algorithms. Considering the multiple 
fault locations estimation in these methods and existence of many laterals in Power Distribution System (PDS) is the drawback of impedance based methods $[28,30]$.

2. Transient based methods, in which discrete Fourier transform or wavelet transform are used to analyze the fault waveform. It is difficult to guarantee the reliability of these methods because of variety of load characteristics and fault causes in PDS [31].

3. Intelligent methods consist of artificial neutral networks (ANN), Expert systems and etc., ANN based methods need to be trained after any change in system and update the network weights, the other drawback with ANN based methods is that in case of complicated networks they become slow and also need to be trained after changes in network [32, 33].

These approaches are mostly centralized and try to locate the exact fault location which is not the case for this research. Recently, with the development of digital sensors and information communication technologies, new FLI techniques can be applied to PDS. Distributed methods such as Multi-Agent Systems (MAS) have received significantly increased attention recently in the community to handle the complex FLI research and development. In [1], D. M. Staszesky et al. presented an implemented FLI system (IntelliTeam $\mathbb{R}$ SG) with team-based structure that use fault current thresholds for fault detection. Presented FLI system requires threshold setting for each installed switch and only support radial power distribution networks.

Authors proposed a distributed multi agent algorithm in previous works, in which agents use local information to locate and isolate the fault in PDS with the presence of Distributed Generation Sources (DGS) [12, 13] . In proposed method, agents are classified as Zone Agents (ZA), Feeder Agents (FA) and Substation Agents (SA), in the bottom-up order and ZAs are responsible for locating the fault and isolating it. Each ZA, according to the rms value of the measured current waveform of itself as well as its neighbors, determines whether there is a fault in its zone or not by monitoring the changes in the current rms values.

The proposed FLI algorithms have been tested through simulation studies and the results are discussed in previous works by the authors, however, its application in a real world power system poses a lot of questions and problems with it. There are various issues such as control and communication between agents and 
power system which were not taken into consideration in the simulation studies. Development of a test system platform, capable of emulating a present day power system and MAS will help in dealing with these challenges.

This chapter presents the development of a platform compromising of meters, agent components, loads integrated with a power system platform to verify applicability of proposed method in locating and isolating the fault using a real world case study. Agents are implemented using power quality meters and rugged computers which communicate with MAS through serial and TCP/IP communication links.

\subsection{Multi-Agent Based Fault Location}

In this section a brief description of multi agent system structure along with the proposed fault location and isolation algorithm are presented.

\subsubsection{MAS Architecture}

As shown in Fig. 10.1, there are three types of agents in the proposed MAS, i.e., ZA, FA and SA. These agents are intelligent units that have problem solving capabilities and can communicate, resolve, coordinate and debate with other agents and make decisions. Zone agents are the lowest level agents which are in contact with their neighbors. Each feeder agent is in charge of a number of zone agents and communicates with the other feeder agents. Substation agent just has a communication link to its feeder agents and also to neighboring SAs.

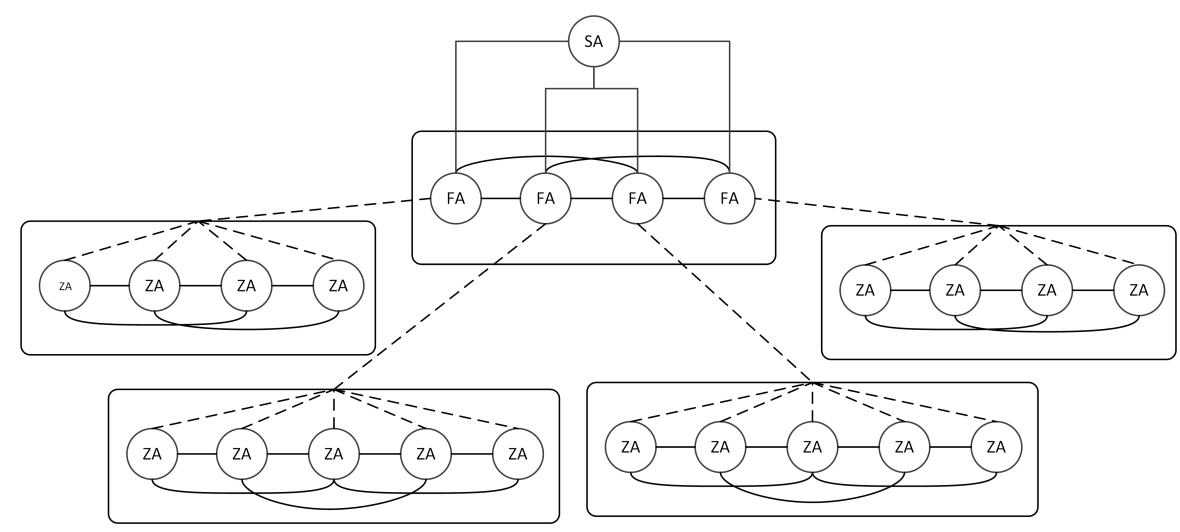

Figure 10.1: MAS ArChitecture 
Distribution feeders are divided into sections by the installed switches along the feeder. The physical switch at the top of each section represents the corresponding zone agent. ZAs can directly communicate with their neighbor ZAs and also corresponding FA. A ZA monitors the status of the corresponding zone voltage, current waveforms and calculates its current usage. ZA contains a load profile estimation function that provides a mechanism for them to predict the future zone load which is required for the Fault Location, Isolation and Restoration (FLIR) algorithms. In Fig. 10.2 the procedure of a ZA operation is illustrated.

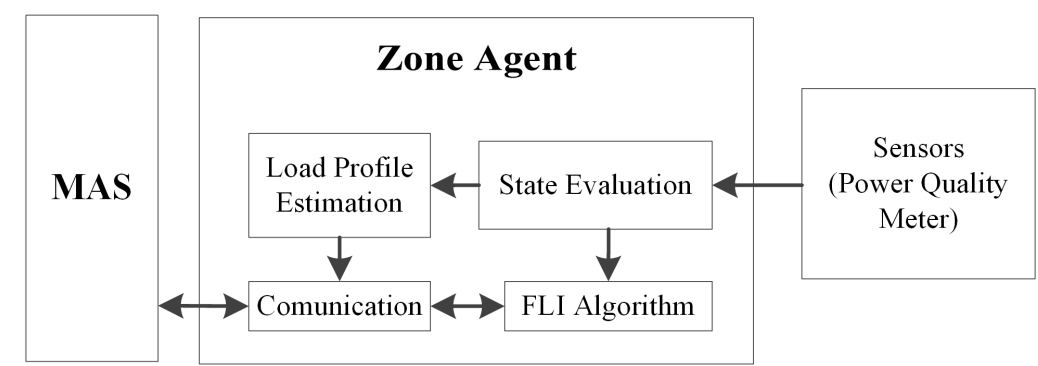

Figure 10.2: Zone Agent (ZA)

\subsubsection{Fault Location Equations}

Each agent has access to its neighbor ZA's data and can calculate the current injected to area between itself and upstream or downstream agents by using the Kirchhoff's law, i.e.

$$
I_{Z_{k}}=\sum_{i=1}^{n} I_{\text {Enterance }}-\sum_{i=1}^{m} I_{\text {Exit }}
$$

where $I_{Z_{k}}$ is the current usage of zone $k$, defined as the difference between the sum of currents entering the zone $\left(I_{\text {Entrance }}\right)$ and currents leaving the zone $\left(I_{\text {Exit }}\right)$. ZAs communicate with their direct neighbors regularly (every 5 minutes) and update this value. By monitoring the trend for $I_{Z}$ changes, each ZA can determine whether there is a fault in its zone or not. Changes are calculated using

$$
I_{Z_{C}}=\left(\frac{\left|I_{Z_{\text {new }}}\right|-\left|I_{Z_{\text {old }}}\right|}{\left|I_{Z_{\text {old }}}\right|}\right) * 100
$$

\subsubsection{Fault Location and Isolation Algorithm}

Fig. 10.3 shows the fault location, isolation algorithm flowchart. In normal operation, agents update their load profile data and also update their knowledge about 
their neighbors. During the fault location process, agents pull out the meters recorded data during the last recloser trial with highest resolution and compare the data with recent normal operation data.

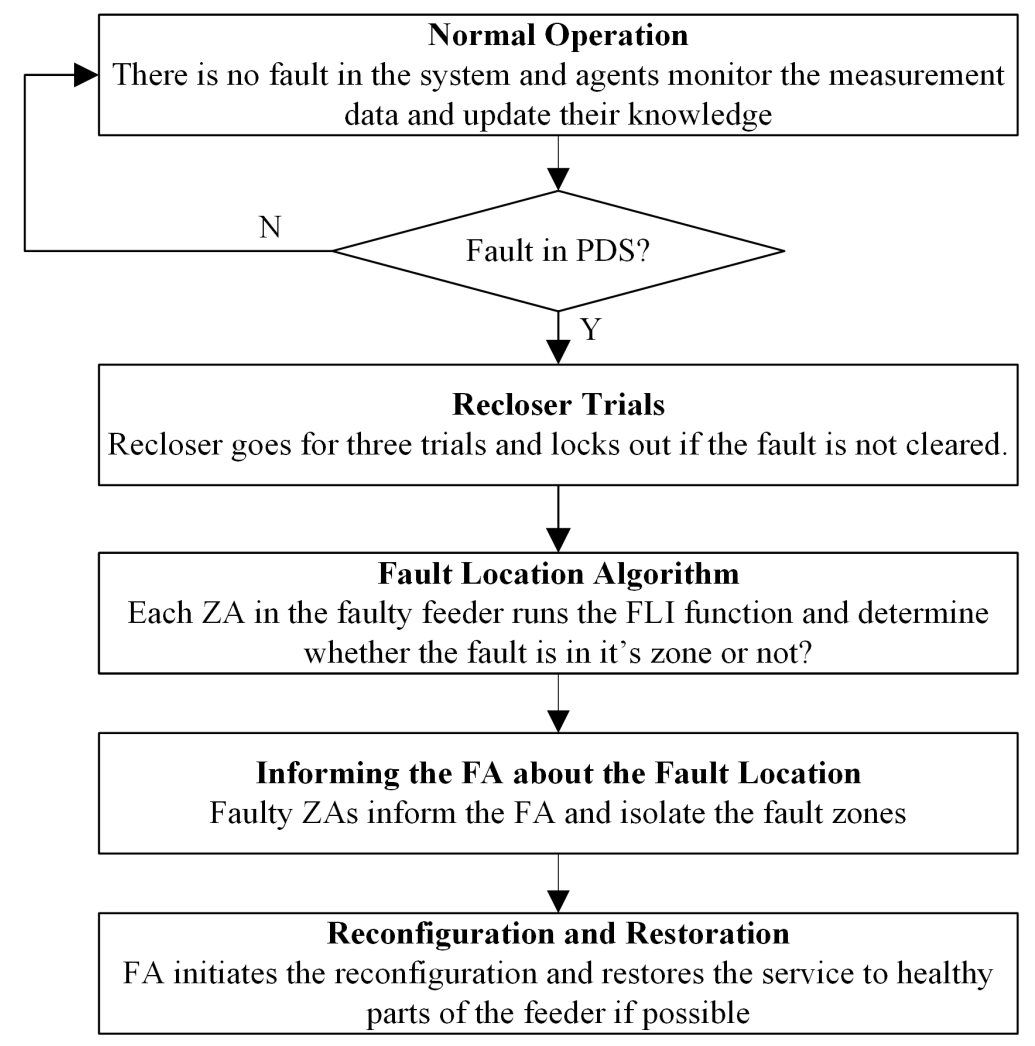

Figure 10.3: FAult locAtion, isolation ALgORITHM FLOWCHART

As a decentralized approach, ZAs just have access to local information and they have no information about the topology. The following algorithm specifies the fault location and isolation steps for ZAs.

(1) Each ZA is aware of its current usage using equation (10.1) and updates it sequentially along with informing its neighbors.

(2) When there is a fault in the feeder and recloser is going for its three trials, FA informs the ZAs to be aware of the trials.

(3) ZAs pull out the higher resolution measured data during the recloser trials period and update their drawn current value using equation (10.1).

(4) After the recloser locks out, agents exchange the recent measured data during trials with their neighbors.

(5) Next, using equation (10.2) ZAs analyze the changes in their drawn current before and during the recloser trials. 
(6) The $Z A_{i}$ with fault sees the highest change in the index, while other zones either see negative or much lower percentage changes in their load profile.

(7) $Z A$ starts communicating to the downstream ZA to confirm isolating the fault.

(8) This process is stopped by $Z A_{i}$, when it confirms with its next neighbor $\left(Z A_{i+1}\right)$ and knows that the fault is between itself and its downstream neighbor.

\subsection{MAS Application Design}

Application design involves a process of modeling the knowledge that will be used by each agent. Agent's responsibilities and ontology are discussed below.

\subsubsection{Zone Agent Responsibilities}

The zone agent represents the zone and communicates with its immediate neighbor zone agents to locate the fault. Each zone agent records the voltage and current waveform with the resolution of 16 samples per cycle and calculates the phasor values. Zone agents calculate and update the indices values introduced in previous section using the current Phasor values (Every minute).

\section{ZA Responsibilities:}

- Register to corresponding FA

- Monitoring system parameters e.g. (V, I, f, ...)

- Communicating to neighbor ZAs

- Running FLI algorithm upon FA request

- Accept incoming registrations from FA \& ZAs

- Responding to registrations from FA \& ZAs

- Storing the information (load profile) 


\subsubsection{Interaction Between Agents}

This subsection describes the general processes of the MAS and the messages used.

\subsubsection{Ontology}

Ontologies are content theories about objects, their properties, and relationships among them that are possible in a specific domain of knowledge. In knowledgebased systems an ontology is that part of the system which specifies what things exist and what is true about them and clarifies the structure of knowledge. The knowledge of each agent is defined by Facts, which represent statements that an agent believes to be true, either about itself or its external environment [78]. Table 10.1 gives a general idea about how these facts are defined.

TABle 10.1: FACTS IN The IMPLEMENTED MAS

\begin{tabular}{|l|c|}
\hline Fact & Description \\
\hline ID & IP Address \\
\hline Switch Status & Open/Closed \\
\hline Corresponding FA IP & IP Address \\
\hline Upstream Neighbors IP & IP Address \\
\hline Downstream Neighbors IP & IP Address \\
\hline Zone's Power Consumption & Watts \\
\hline
\end{tabular}

Each agent type has its own responsibilities and settings. The agent creation process involves the creation of generic agent of each type i.e. (ZA, FA, SA). This is done by attributing certain tasks, rules, coordination protocols and status of each agent. In this work, FIPA compliant coordination protocols are attributed to all agents [58].

The data packets that ZAs send to their corresponding neighbors have 13 elements. These elements are time tagged three phases voltage and current phasors, i.e.

$$
\left(V_{a b c}, I_{a b c}, \varphi_{a b c}^{V}, \varphi_{a b c}^{I}, t\right)
$$

Where $\varphi$ is the phase angle. 


\subsubsection{Communication}

When a fault situation occurs and before the recloser runs for the three trials, FA sends a REQUEST message to its ZAs and asks them to monitor their zones for faults. When the ZA receives the REQUEST message, it pulls out the higher resolution data continuously (up to 512 samples per cycle) and updates the index values during the recloser trials.

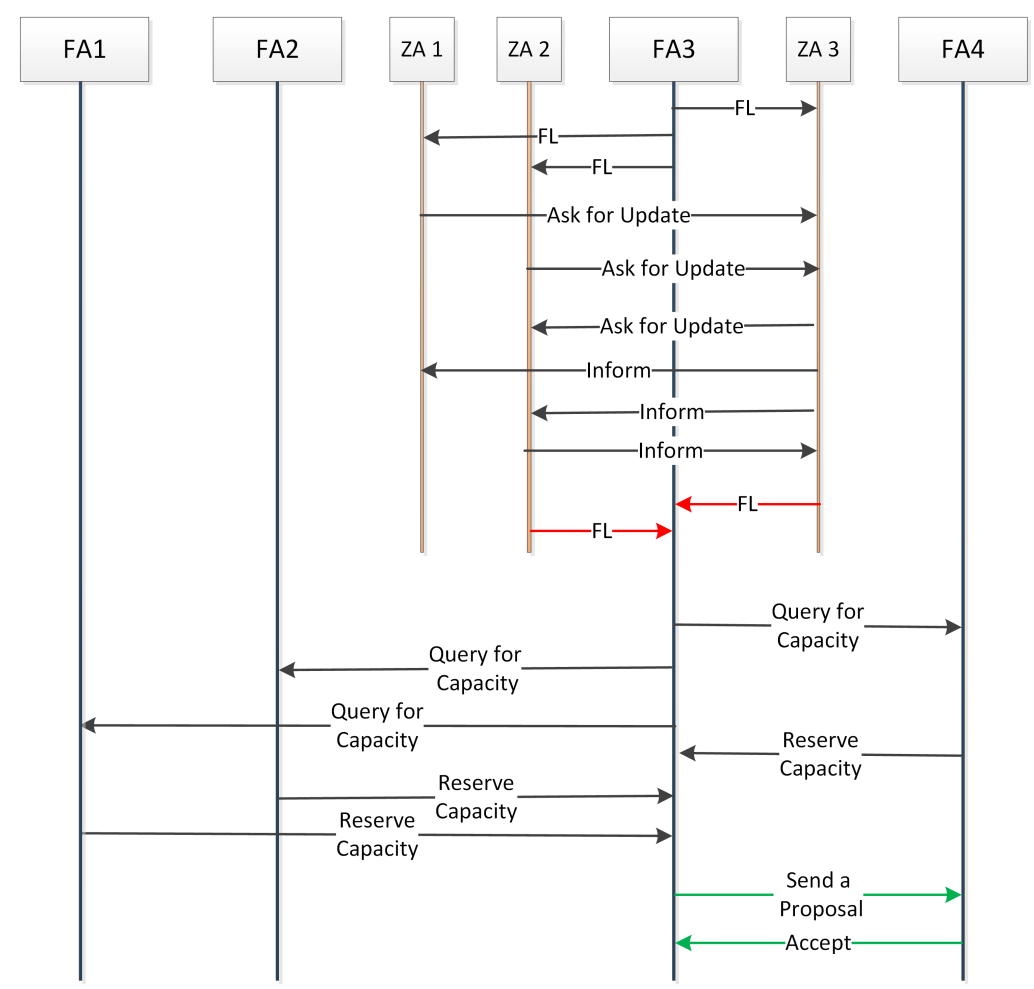

Figure 10.4: Agents Communication for FLi

After the recloser locks out, ZAs running the FLI algorithm can decide whether the fault is in their zones or not and inform the FA about their zone status. Fig. 10.4 shows the communications when there is a fault between ZA2 and ZA3. FLI process starts upon FA's request and terminates as ZAs report the fault location. FA will start the restoration process after receiving the fault location signal.

\subsection{Implementation of Proposed FLI System}

The MAS is deployed on a real hardware testbed built by the Advanced Power and Electricity Research Center (APERC) group. The testbed provides a validation and demonstration platform for the distributed MAS-based fault location and 
isolation algorithm. The implementation details on components, communication media and software programs are discussed below.

\subsubsection{Components}

An agent is a computational entity that perceives its environment through sensors. MAS is composed of several of this kind of agents interacting with each other to achieve a global goal. Fig 10.5 shows the implementation of an agent consisting of a SEL-735 as a sensor and a Nuvo-1000 as a computation unit described in this section.

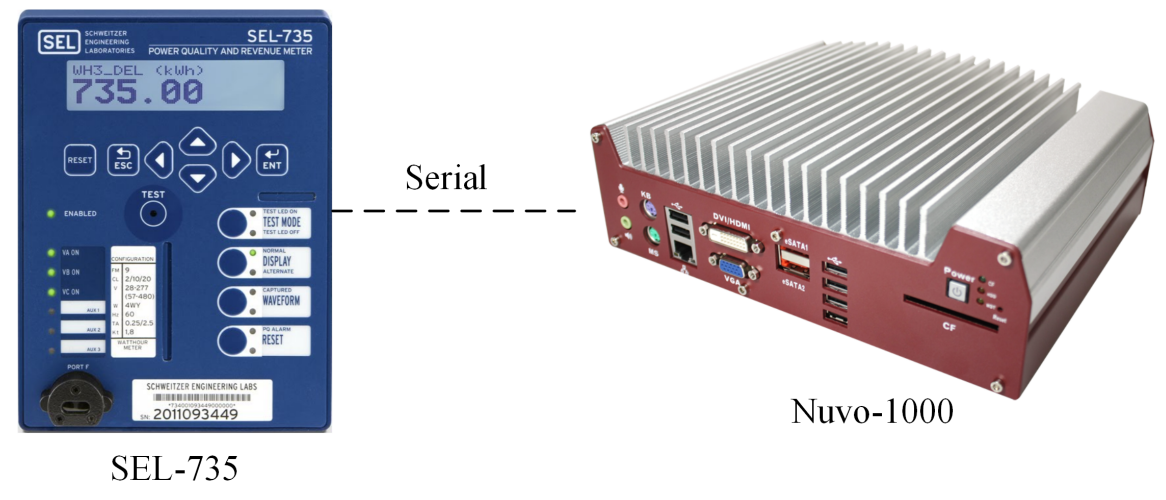

Figure 10.5: Agent Implementation

\subsubsection{SEL}

SEL-734 and 735 are 32-bit microprocessor-based power quality meters with a combination of recording, automation, and communications capabilities. Meter shall meet IEC 61000-4-30 power quality accuracy for voltage, current, power, harmonics, and flicker. It simultaneously captures six-channel 512 samples-per-cycle waveforms with programmable logic triggers. Table 10.2 provides more information about the device specification [79].

The SEL-735 has three normal access levels: Access Level 1 (ACC), Access Level 2 (2AC), and Access Level E (EAC). Access level 1 is to view meter settings and quantities, Access level 2 is for configuring all settings and control functions of the SEL-735. From these three access levels, the user can fully configure and 
Table 10.2: Specification of SEL 735 Power Quality Meters

\begin{tabular}{|l|l|}
\hline Accuracy & ANSI and IEC Class 0.2 accuracy \\
\hline Recording & at least for 140 days at five-minute intervals \\
\hline Waveform Capture & 512 samples-per-cycle \\
\hline Inputs/Outputs & 4 digital inputs / 4 solid-state, or analog outputs \\
\hline Communication & up to 10 simultaneous communications sessions \\
\hline Protocols & Modbus $\AA$, IEC 61850, DNP3, MV-90, ASCII \\
\hline Temperature & $-40^{\circ} \mathrm{C} \sim 85^{\circ} \mathrm{C}$ \\
\hline
\end{tabular}

operate the meter. User can gain access to the access levels through certain communications protocols. For example, user can write to some registers via DNP and Modbus ${ }^{\circledR}$ after gaining EAC access through the same communications channel. Table 10.3 shows some sample commands to communicate with the SEL-735 meter.

Table 10.3: SEL-735 Command Summary

\begin{tabular}{|c|c|l|}
\hline Access Level & Command & Description \\
\hline 0 & ACC & Move to Access Level 1 \\
0 & ID & Compressed ASCII Fast Meter ID \\
\hline 1 & 2 AC & Move to Access Level 2 \\
1 & EVE & Latest event report, 16 samples per cycle \\
1 & MET & Display instantaneous metering data \\
1 & MET RMS & Display rms metering data \\
1 & TIM & Show time \\
\hline E & $\ldots$ & is for setting the meter parameters \\
\hline C & $\ldots$ & is for diagnosing troublesome installations \\
\hline
\end{tabular}

\subsubsection{Processing Units}

Nuvo-1000 controller offers extraordinary performance for arithmetic-intensive applications, while its fan-less design provides superb reliability and durability. Nuvo1000 integrates versatile I/O interfaces in a compact chassis. It provides up to 5 Gigabit Ethernet ports and 2 eSATA ports, which are particularly useful for multi-agent applications. The specification of Nuvo-1003S-620M, which are used to handle the agent responsibilities is shown in Table 10.4 [80]. 
TABle 10.4: Specification of Nuvo-1003S

\begin{tabular}{|c|c|}
\hline Processor & Intel (R) Core ${ }^{\mathrm{TM}} \mathrm{i} 7-620 \mathrm{M}(2.66 \mathrm{GHz}, 4 \mathrm{MB})$ \\
\hline Memory & up to 4GB DDR3 $1066 \mathrm{MHz}$ SDRAM \\
\hline Power Consumption & Maximal: 3.1A @ 19V (58.9W) \\
\hline Operating Temp & $-25^{\circ} \mathrm{C} \sim 70^{\circ} \mathrm{C}$ \\
\hline Ethernet & 5x Intel (R) 82574L GbE ports \\
\hline Serial Port & 1x P RS-232 \& 3x RS-232 (COM1-COM4) \\
\hline USB & 6x USB 2.0 ports \\
\hline
\end{tabular}

\subsubsection{Communication Media}

Serial and TCP/IP communication media have been used to implement the MAS communication. Serial connection is implemented to establish the connection between each SEL-735 and the Nuvo controller. All messaging exchanges among agents are established via the Transmission Control Protocol/Internet Protocol or TCP/IP. The TCP/IP server is implemented in MATLAB [65] to connect agents to their neighboring agents. Table 10.5 shows the setting for each of the implemented communication media.

Table 10.5: Communication Ports Settings

\begin{tabular}{|l|c||l|c|}
\hline \multicolumn{2}{|c||}{ Serial } & \multicolumn{2}{c|}{ TCP/IP } \\
\hline Port & $C O M_{n}$ & RemotePort & remote port \\
\hline BaudRate & 4800 & RemoteHost & remote host \\
\hline Terminator & $\#$ & Terminator & 'LF' \\
\hline Status & open/closed & NetworkRole & client/server \\
\hline TransferStatus & idle & TransferStatus & idle \\
\hline BytesAvailable & $\mathrm{X}$ & BytesAvailable & $\mathrm{X}$ \\
\hline ValuesReceived & $\mathrm{X}$ & ValuesReceived & $\mathrm{X}$ \\
\hline ValuesSent & $\mathrm{X}$ & ValuesSent & $\mathrm{X}$ \\
\hline
\end{tabular}

FA operates as TCP/IP server for its ZAs and ZAs are FA's clients. Also, each ZA operates as a TCP/IP server for its downstream neighbor.

\subsubsection{Software Program}

MATLAB is installed on Nuvo-1000 machine for all agents. Agents tasks and behaviors are programmed into several functions. These function are responsible 
for running the agents tasks and negotiations. Software program functions are summarized below:

- Establishing serial connection to the meter

- Establishing the TCP/IP connection to corresponding FA and neighboring ZAs

- Writing registers to SEL meter (To achieve access to SEL and load settings)

- Reading the Serial port buffer continuously

- Filtering the received data

- Communicating (Send/Receive data) to neighboring ZAs

- Running the FLI algorithm upon FA request

These functions are programmed on each agent and run independently.

\subsubsection{Results and Discussion}

The proposed multi-agent system is implemented on a test bed as a simplified distribution circuit that comprises: a $4 \mathrm{~kW}$ source, a $2 \mathrm{~kW}$ auto transformer, 9 Solid State Relays (25 A), and 3 agents (one FA and two ZAs). Relays are connected to SEL meters with wires. In Figs. ?? a 3 phase fault is applied between ZA1 and ZA2.

Figs. 10.8 shows the rms currents measured by the ZA1 for the period of fault occurrence and re-closer operations.

Recloser sees an increase in feeder current and goes for three trials. The calculated index $\left(I_{Z_{1}}\right)$ for the ZA1 in the implemented circuit rise from 0 to $50 \%$ after the fault occurs at $t=9 s$, the index for ZA2 decreases $25 \%$. ZA1 and ZA2 both see this changes in their zones current and decide to isolate the fault zone along with informing the FA about fault location. 


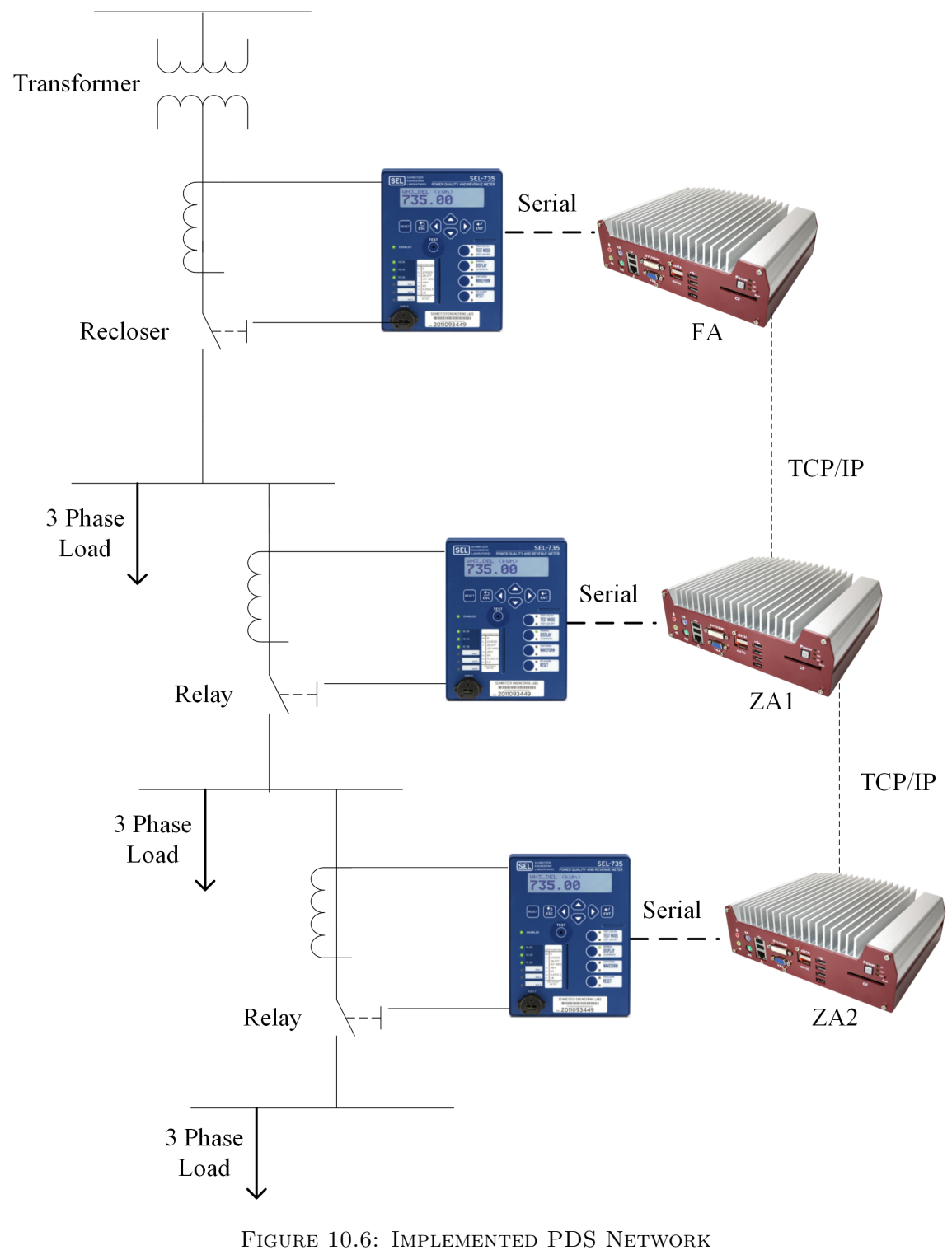




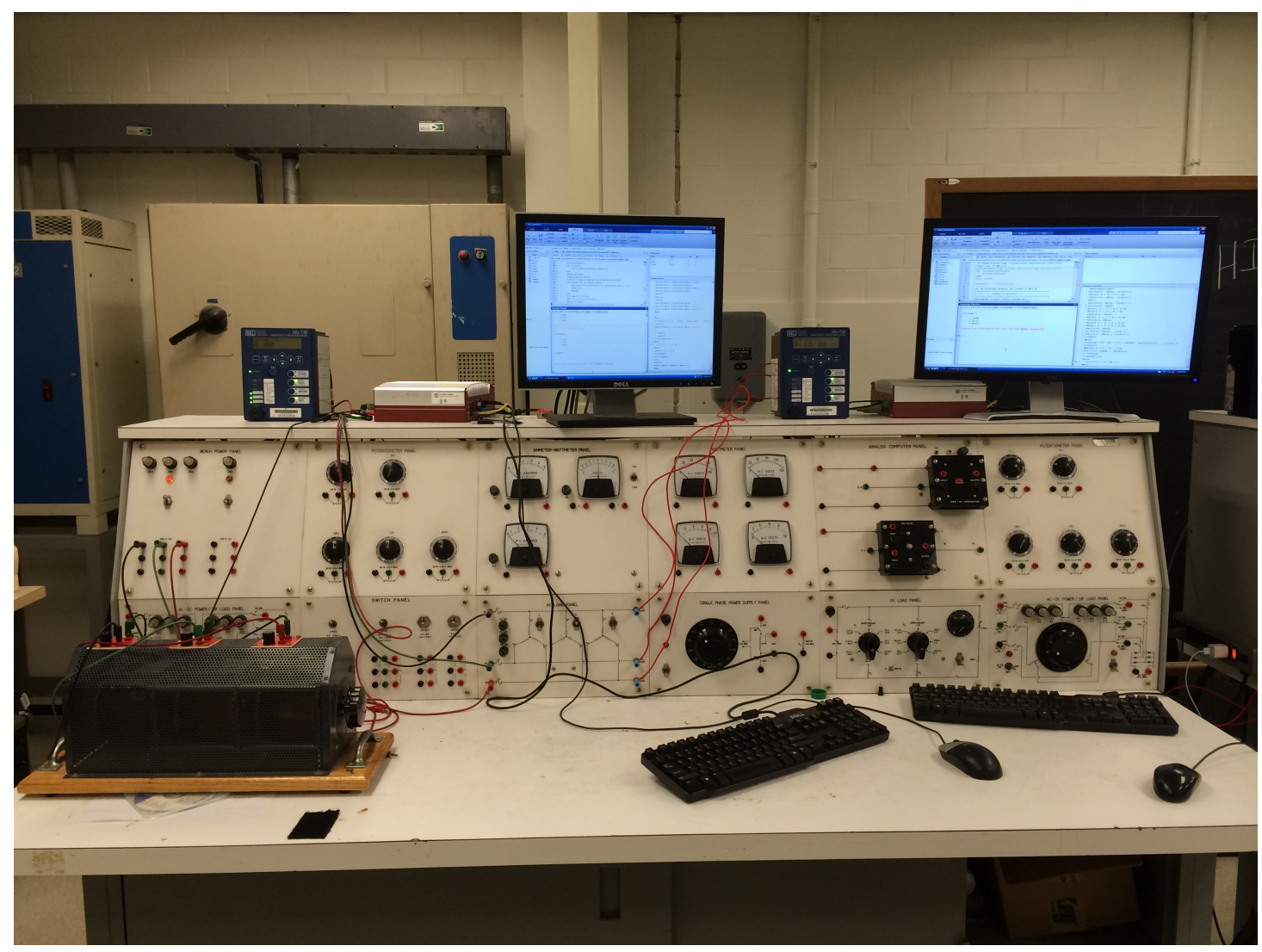

Figure 10.7: LABORATORY Implementations

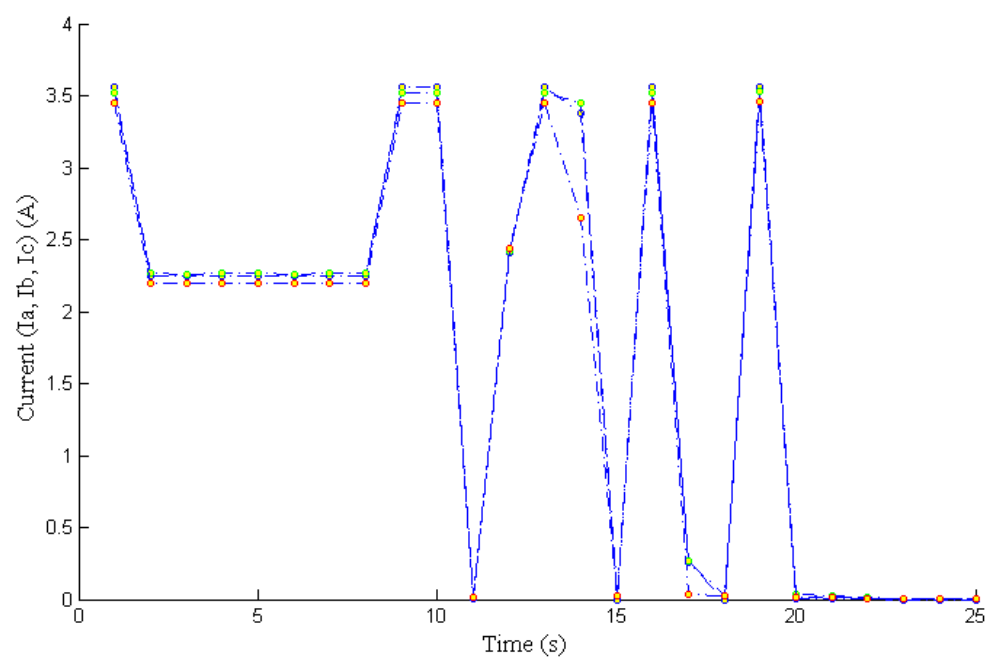

Figure 10.8: Measured Fault current and recloser Operation 


\section{Chapter 11}

\section{Conclusions and Future Work}

\subsection{Conclusions}

In this dissertation, a new multi-agent system design with learning capability $(Q$-Learning) for power distribution systems automation is presented. The main contributions of this work are:

- Distributed fault location and isolation algorithm:

Fault location and isolation is the first step of PDS automation system and faster FLI means faster service restoration and shorter outage time. Restoration process cannot be initiated unless the fault location is determined and faulty section is isolated. Therefore, it is important to locate and isolate the fault as fast as possible and preferably using a non-centralized approach. Proposed FLI algorithm in this dissertation is totally distributed and does not require any threshold setting and adjustments. It is important to perform the FLI using a distributed method so that no single point of failure exist in the system. With increasing penetration of distributed generation sources into power distribution systems, it is necessary to consider the effect of DG penetration on FLI applications too. Proposed FLI algorithm is one of the few available algorithms that can locate the fault in power distribution systems with DG penetrations.

- Hybrid approach for reconfiguration and service restoration:

Once the fault is isolated, the optimal load restoration decision can be obtained using different techniques. Multiagent systems is one of the most 
popolar approaches for restoration optimization. Both centralized and distributed MAS have been used to find the optimal configuration for restoration. Due to complexity and expansion of present-day distribution systems, centralized systems tend to be inadequate because of dependency on powerful central computing facilities to handle huge amount of data with high communication capabilities, which can lead to a single point of failure. But centralized methods have the advantage of having an overview on all the system parameters and can find the optimal reconfiguration. On the other hand, distributed MAS can overcome the disadvantages of centralized approaches because of their decentralized data processing, which leads to efficient task distribution and eventually causing faster operation and decision making process, but they have the disadvantage of local optima because of availability of only local data. This work presented a hybrid MAS for restoration that takes the advantages of both centralized and decentralized approaches. While agents are designed to make local decisions for restoration, they use the control center data in case of availability. Using the proposed hybrid topology, agents can make the optimal decisions based on the available local data and the data from control center. In the designed MAS framework, agents communicate fewer number of messages and use their learning knowledge for decision making which can reduce the computational burden significantly and improve both cost and speed of distribution automation.

- Real-time MAS and power system simulation:

Existing electric power simulation tools accurately model power systems of the past, which were controlled without significant communication elements. It has not been necessary to simulate communication in order to accurately model these electric power systems. However, over the last decade, power systems have begun to conclude that the use of network communication based on Internet standards is a natural choice to improve on the protection and control systems. Multi agent system frameworks can hide the complexity involved in the combined simulation of power system and its communications, making it easy for users to design new power scenarios involving communication.

The combined simulation of electric power and communication systems is rare in the literature. In this work authors developed a module written in 
MATLAB S-functions to allow agents to model behavior in a power system and to communicate over a network. The advantage of developed CoSimulation model is that both power system model and MAS model softwares run in real-time. Therefore, there is no need to interface and synchronize two different softwares. In our model, the amount of time between synchronization points between the two models has a fixed length.

- Learning based decision making for restoration:

Although the agents in MAS can be programmed with behaviors designed in advance, it is also necessary that they learn new behaviors online from thier experiences, such that the performance of the agents and MAS gradually improves. In this dissertation, the novel concept and detailed learning methodology is introduced to help agents in their decision making process. Although agents have the learning capability, they need to take actions to learn from them. In a real world power system fault events do not happen frequently and therefore it takes a long time for agents to gain knowledge about different scenarios. One possible solution to help agents learn faster is initialization of the agents knowledge by simulating different possible scenarios in a software environment and initialize agents knowledge about the consequences of possible actions.

Using the learning technology, agents in PDS will be expert decision makers over time and can make more accurate and comprehensive decisions for a self-healing distribution system.

- Distributed load shedding algorithm:

Load shedding is the term used to describe the deliberate switching off of electrical supply to parts of the electricity network, and hence to the customers in those areas. This practice is rare, but is a core part of the emergency management of all electricity networks. When there is a shortfall in the electricity supply, there can be a need to reduce demand very quickly to an acceptable level so that the power can be restored to as many higher priority customers as possible.

In this dissertation a novel automatic distributed load shedding algorithm is presented that operates based on the predicted load profiles. In proposed design load shedding decisions are made locally and in the ZA location. The advantage of the proposed design is that it sheds the minimum amount of 
the load while it considers the predicted load profiles for a 4 hours window to prevent short term restorations.

To predict the future load profiles, active learning based load prediction method which works based on fuzzy inferential rules is used that can predicts the load using the available data and learns to improve the prediction accuracy over time using the real-time data.

- Hardware implementation of a distributed FLI in a laboratory platform:

Although the designed MAS was tested through a few simulation case studies and the validity and effectiveness of the proposed design have been demonstrated by applying it to a practical distribution network (WVSC). Application of the developed algorithms always poses as many problems and questions as it may solve.

There are various issues such as synchronization and coordination of agents behavior, which can not be taken into consideration in the current power system FLIR studies. Also, there are many issues related to control and communication between agents and power system.

To verify the applicability of the proposed fault location and isolation algorithms, a distributed MAS platform has been implemented in the APERC laboratory. The developed test system platform is capable of emulating a present day power system and implemented agents will help in dealing with the real-world challenges. It will also help in conducting research on the various technical issues pertaining to the FLIR, distributed generation integration, communications and power system. This platform also can help educate future engineers and make them more knowledgeable about these new system operation concepts.

\subsection{Future Work}

While this dissertation has discussed the design of a MAS for power distribution system automation, many opportunities for extending the scope of this dissertation remain. The following main items can be suggested as future work:

- Using multi agent system technology for Micro Grids control and management: 
Microgrid is a possible future energy system paradigm, formed by the interconnection of small, modular generation (micro-turbines, fuel cells, PV, etc.), together with storage devices (flywheels, energy capacitors and batteries) and controllable loads at low voltage distribution systems. Such systems can be operated interconnected to the power grid, or islanded, if disconnected from the grid.

Operation of modern power systems becomes extremely complex with the introduction of distributed power generation, load control, market operation, increasing complexity of distribution networks, and a large number of interconnections. Consequently, real-time control and management of modern power systems has become more complex. Therefore, smart control and energy management systems that are different from those commonly used in the past are necessary for efficient operation of modern power systems. The distributed nature and potential for modeling autonomous decision making entities in solving complex problems motivates the use of multiagent system for the control and management of microgrids. Proposed multiagent system has the autonomous decision making agents that can be modified for real-time control and management of a microgrid.

- Developing a Demand Side Management (DSM) system into the proposed MAS system:

Demand side management plays an important role in smart grid operation. Controlling energy demand can reduce the peak demand, and reshape the load profile, which increases the grid sustainability by reducing the overall cost and carbon emission level. This will lead to the avoidance of construction of an under-utilized electrical infrastructure in terms of generation capacity, transmission lines and distribution networks. Generation scheduling determines the schedule of generation units within the microgrid subject to operational constraints of the microgrid and generation units. Significant cost savings can be achieved by optimizing the generation schedules over the planning horizon. Intelligent agents with forecasting and communication capabilities can be a possible solution for DSM.

- Hardware implementation of the proposed restoration and load shedding algorithms in a real-world application:

Although proposed restoration and load shedding algorithms have been tested through simulation studies and the results are discussed by the authors, 
however, its application in a real world implementation always poses a lot of questions and problems with it. There are various issues such as control and communication between agents and power system which were not always taken into consideration in the simulation studies. Development of a test system platform, capable of emulating a present day power system and MAS will help in dealing with these challenges. 


\section{Bibliography}

[1] D.M. Staszesky, D. Craig, and C. Befus. Advanced feeder automation is here. Power and Energy Magazine, IEEE, 3(5):56-63, Sept 2005.

[2] J.R. Aguero. Applying self-healing schemes to modern power distribution systems. In Power and Energy Society General Meeting, 2012 IEEE, pages $1-4,2012$.

[3] Revelle W. and Galland E. Lakeside Court: An Energy-efficient House. http://revelle.net/lakeside, California, 2008.

[4] National Energy Technology Laboratory. "a vision for the modern grid". 2007. URL Available:http://www.netl.doe.gov/smartgrid/.

[5] S.M. Amin and B.F. Wollenberg. Toward a smart grid: power delivery for the 21st century. IEEE Power and Energy Magazine, 3(5):34-41, 2005.

[6] European Commission. "strategic research agenda for europe's electricity networks of the future". 2007. URL Available:http://www.smartgrids. $\mathrm{eu} /$.

[7] L. Xu, Mo-Yuen Chow, J. Timmis, and L.S. Taylor. Power distribution outage cause identification with imbalanced data using artificial immune recognition system (airs) algorithm. IEEE Trans. Power Syst., 22(1):198-204, 2007.

[8] Disturbance Analysis Working Group (DAWG) group. "north american electric reliability council (nerc)". URL Available:http://www.nerc.com/.

[9] J. Ghorbani, Y. Fallah, M.A. Choudhry, and A. Feliachi. Investigation of communication media requirements for self healing power distribution systems. In Energytech, 2013 IEEE, 2013. 
[10] M.J. Ghorbani, M.A. Choudhry, and A. Feliachi. Multi agent system design for self-healing power distribution systems. IEEE Trans. Smart Grids, Submitted on Oct 2013.

[11] J. Ghorbani, Y. Fallah, M.A. Choudhry, and A. Feliachi. Hybrid multi agent approach for power distribution system restoration. In Energytech, 2013 IEEE, 2013.

[12] J. Ghorbani, M.A. Choudhry, and A. Feliachi. Real-time multi agent system modeling for fault detection in power distribution systems. In North American Power Symposium (NAPS), 2012, pages 1-6, 2012.

[13] J. Ghorbani, M.A. Choudhry, and A. Feliachi. Fault location and isolation using multi agent systems in power distribution systems with distributed generation sources. In Innovative Smart Grid Technologies (ISGT), 2013 IEEE PES, pages 1-6, 2013.

[14] J. Ghorbani, M.A. Choudhry, and A. Feliachi. A mas learning framework for power distribution system restoration. In IEEE PES Transmission \&6 Distribution Conference, 2014, pages 1-6, Submitted on July 2013.

[15] L.H. Chiang, E.L. Russell, and R.D. Braatz. Fault Detection and Diagnosis in Industrial Systems. Springer publications, 2001.

[16] K.M. Hopkinson, K.P. Birman, R. Giovanini, D.V. Coury, X. Wang, and J.S. Thorp. Epochs: integrated commercial off-the-shelf software for agentbased electric power and communication simulation. In Simulation Conference, 2003. Proceedings of the 2003 Winter, volume 2, pages 1158-1166 vol.2, 2003.

[17] K. Hopkinson, Xiaoru Wang, R. Giovanini, J. Thorp, K. Birman, and D. Coury. Epochs: a platform for agent-based electric power and communication simulation built from commercial off-the-shelf components. IEEE Trans. Power Syst., 21(2):548-558, 2006.

[18] D. Cartes G. Morejon, L. Sun. Corba implementation for interfacing software agents with virtual test bed. In ASNE Reconfiguration and Survivability Symposium 2005, Atlantic Beach, Florida, 2005.

[19] I. Zabet and M. Montazeri. Implementing cooperative agent-based protection and outage management system for power distribution network control. In 
Power Engineering and Optimization Conference (PEOCO), 2010 4th International, pages 318-324, 2010.

[20] J.G. Gomez-Gualdron, M. Velez-Reyes, and L.J. Collazo. Self-reconfigurable electric power distribution system using multi-agent systems. In Electric Ship Technologies Symposium, 200\%. ESTS '0\%. IEEE, pages 180-187, 2007.

[21] T. Nagata and H. Sasaki. A multi-agent approach to power system restoration. IEEE Trans. Power Syst., 17(2):457-462, 2002.

[22] T. Nagata, Y. Tao, K. Kimura, H. Sasaki, and H. Fujita. A multi-agent approach to distribution system restoration. In Circuits and Systems, 2004. MWSCAS '04. The 2004 47th Midwest Symposium on, volume 2, pages II333-II-336 vol.2, 2004.

[23] Dayong Ye, Minjie Zhang, and D. Sutanto. A hybrid multiagent framework with q-learning for power grid systems restoration. IEEE Trans. Power Syst., 26(4):2434-2441, 2011.

[24] J.M. Solanki, S. Khushalani, and N.N. Schulz. A multi-agent solution to distribution systems restoration. IEEE Trans. Power Syst., 22(3):1026-1034, 2007.

[25] Fenghui Ren, Minjie Zhang, D. Soetanto, and XiaoDong Su. Conceptual design of a multi-agent system for interconnected power systems restoration. IEEE Trans. Power Syst., 27(2):732-740, 2012.

[26] Li Liu, Kevin P. Logan, D.A. Cartes, and S.K. Srivastava. Fault detection, diagnostics, and prognostics: Software agent solutions. Vehicular Technology, IEEE Transactions on, 56(4):1613-1622, 2007.

[27] S. Alwala, A. Feliachi, and M.A. Choudhry. Multi agent system based fault location and isolation in a smart microgrid system. In Innovative Smart Grid Technologies (ISGT), 2012 IEEE PES, pages 1-4, 2012.

[28] R.H. Salim, M. Resener, A.D. Filomena, K. Rezende Caino de Oliveira, and A.S. Bretas. Extended fault-location formulation for power distribution systems. IEEE Trans. Power Del., 24(2):508-516, 2009.

[29] R.H. Salim, K. de Oliveira, A.D. Filomena, M. Resener, and A.S. Bretas. Hybrid fault diagnosis scheme implementation for power distribution systems automation. IEEE Trans. Power Del., 23(4):1846-1856, 2008. 
[30] Yun-Seok Ko, Tae-Ku Kang, Hak-Yeol Park, Ho-Yong Kim, and Hyo-Seong Nam. The frtu-based fault-zone isolation method in the distribution systems. IEEE Trans. Power Del., 25(2):1001-1009, 2010.

[31] M. Michalik, W. Rebizant, M. Lukowicz, Seung-Jae Lee, and Sang-Hee Kang. High-impedance fault detection in distribution networks with use of waveletbased algorithm. IEEE Trans. Power Del., 21(4):1793-1802, 2006.

[32] D. Thukaram, H. P. Khincha, and H. P. Vijaynarasimha. Artificial neural network and support vector machine approach for locating faults in radial distribution systems. IEEE Trans. Power Del., 20(2):710-721, 2005.

[33] Shyh-Jier Huang. Application of immune-based optimization method for fault-section estimation in a distribution system. IEEE Trans. Power Del., 17(3):779-784, 2002.

[34] Yann-Chang Huang. Fault section estimation in power systems using a novel decision support system. IEEE Trans. Power Syst., 17(2):439-444, 2002.

[35] Wen-Hui Chen, Shun-Hung Tsai, and Hsien-I Lin. Fault section estimation for power networks using logic cause-effect models. Power Delivery, IEEE Transactions on, 26(2):963-971, 2011.

[36] Yinliang $\mathrm{Xu}$ and Wenxin Liu. Novel multiagent based load restoration algorithm for microgrids. IEEE Trans. Smart Grids, 2(1):152-161, 2011.

[37] R. Perez-Guerrero, G.T. Heydt, N.J. Jack, B.K. Keel, and A.R. Castelhano. Optimal restoration of distribution systems using dynamic programming. IEEE Trans. Power Del., 23(3):1589-1596, 2008. ISSN 0885-8977. doi: 10.1109/TPWRD.2007.916112.

[38] T. Taylor and D. Lubkeman. Implementation of heuristic search strategies for distribution feeder reconfiguration. IEEE Trans. Power Del., 5(1):239-246, 1990 .

[39] K.L. Butler-Purry and N. D R Sarma. Self-healing reconfiguration for restoration of naval shipboard power systems. IEEE Trans. Power Syst., 19(2): 754-762, 2004. ISSN 0885-8950. doi: 10.1109/TPWRS.2003.821431.

[40] Qin Zhou, D. Shirmohammadi, and W.-H.E. Liu. Distribution feeder reconfiguration for service restoration and load balancing. IEEE Trans. Power Syst., 12(2):724-729, 1997. ISSN 0885-8950. doi: 10.1109/59.589664. 
[41] C.P. Nguyen and A.J. Flueck. Agent based restoration with distributed energy storage support in smart grids. Smart Grid, IEEE Transactions on, 3(2): 1029-1038, 2012.

[42] Chen-Ching Liu, Seung-Jae Lee, and S.S. Venkata. An expert system operational aid for restoration and loss reduction of distribution systems. Power Systems, IEEE Transactions on, 3(2):619-626, 1988.

[43] A.L. Morelato and A. Monticelli. Heuristic search approach to distribution system restoration. Power Delivery, IEEE Transactions on, 4(4):2235-2241, 1989.

[44] S. Das, S. Bose, S. Pal, N.N. Schulz, C.M. Scoglio, and B. Natarajan. Dynamic reconfiguration of shipboard power systems using reinforcement learning. IEEE Trans. Power Syst., 28(2):669-676, 2013.

[45] R. Perez-Guerrero, G.T. Heydt, N.J. Jack, B.K. Keel, and A.R. Castelhano. Optimal restoration of distribution systems using dynamic programming. IEEE Trans. on Power Delivery, 23(3):1589-1596, 2008.

[46] Ye Yan, Yi Qian, H. Sharif, and D. Tipper. A survey on smart grid communication infrastructures: Motivations, requirements and challenges. Communications Surveys Tutorials, IEEE, 15(1):5-20, 2013.

[47] F. Bouchhima, M. Briere, G. Nicolescu, M. Abid, and E.-M. Aboulhamid. A systemc/simulink co-simulation framework for continuous/discrete-events simulation. In Behavioral Modeling and Simulation Workshop, Proceedings of the 2006 IEEE International, pages 1-6, 2006.

[48] Hua Lin, S.S. Veda, S.S. Shukla, L. Mili, and J. Thorp. Geco: Global eventdriven co-simulation framework for interconnected power system and communication network. Smart Grid, IEEE Transactions on, 3(3):1444-1456, 2012.

[49] Kun Zhu, M. Chenine, and L. Nordstrom. Ict architecture impact on wide area monitoring and control systems' reliability. Power Delivery, IEEE Transactions on, 26(4):2801-2808, 2011.

[50] Christopher JCH Watkins and Peter Dayan. Q-learning. Machine learning, 8(3-4):279-292, 1992. 
[51] R. Faranda, A. Pievatolo, and E. Tironi. Load shedding: A new proposal. Power Systems, IEEE Transactions on, 22(4):2086-2093, 2007.

[52] S. A. Nirenberg, D. A. McInnis, and K. D. Sparks. Fast acting load shedding. Power Systems, IEEE Transactions on, 7(2):873-877, 1992.

[53] M. Parniani and A. Nasri. Scada based under frequency load shedding integrated with rate of frequency decline. In Power Engineering Society General Meeting, 2006. IEEE, pages 6 pp.--, 2006.

[54] H. Seyedi and M. Sanaye-Pasand. New centralised adaptive load-shedding algorithms to mitigate power system blackouts. Generation, Transmission Distribution, IET, 3(1):99-114, 2009.

[55] H. Keshtkar, J. Solanki, and S.K. Solanki. Application of phev in load frequency problem of a hybrid microgrid. In North American Power Symposium (NAPS), 2012, pages 1-6, 2012.

[56] $S$ \& $C$ Electric Company, IntelliTeam® $S G$ Automatic Restoration System. http://www.sandc.com/products/automation-control/ intelliteam.asp.

[57] S.D.J. McArthur, E.M. Davidson, V.M. Catterson, A.L. Dimeas, N.D. Hatziargyriou, F. Ponci, and T. Funabashi. Multi-agent systems for power engineering applications 2014;part i: Concepts, approaches, and technical challenges. Power Systems, IEEE Transactions on, 22(4):1743-1752, 2007.

[58] FIPA ACL Specifications. Standards Committee of the IEEE Computer Society. available at http:www.fipa.org, 2013.

[59] V.C. Gungor, D. Sahin, T. Kocak, S. Ergut, C. Buccella, C. Cecati, and G.P. Hancke. Smart grid technologies: Communication technologies and standards. IEEE Trans. on Industrial Informatics, 7(4):529-539, 2011.

[60] D.M. Staszesky. Use of virtual agents to effect intelligent distribution automation. In IEEE Power Engineering Society General Meeting, 2006., pages 6 pp.-, 2006. doi: 10.1109/PES.2006.1709593.

[61] William H . Kersting. Distribution system modeling and analysis. In Second Edition (Electric Power Engineering Series), page 45, Boca Raton, Florida, 2006. Taylor \& Francis Group. 
[62] L. Busoniu, R. Babuska, and B. De Schutter. A comprehensive survey of multiagent reinforcement learning. IEEE Trans. Systems, Man, and Cybernetics, Part C: Applications and Reviews, 38(2):156-172, 2008. ISSN 1094-6977. doi: 10.1109/TSMCC.2007.913919.

[63] L. Busoniu, R. Babuska, and B. De Schutter. Multi-agent reinforcement learning: A survey. In Control, Automation, Robotics and Vision, 2006. ICARCV '06. 9th International Conference on, pages 1-6, 2006.

[64] M. Hemmati, N. Sadati, and M. Nili. Towards a bounded-rationality model of multi-agent social learning in games. In Intelligent Systems Design and Applications (ISDA), 2010 10th International Conference on, pages 142-148, 2010.

[65] MATLAB Simulink. version 7.14 .0 (R2012a). The MathWorks Inc., Natick, Massachusetts, 2012.

[66] International T CYME (CYMDIST) and D. version 2012. Cooper Power Systems, Houston, Texas, 2013.

[67] M.M. Nordman and M. Lehtonen. An agent concept for managing electrical distribution networks. IEEE Trans. Power Del., 20(2):696-703, 2005.

[68] M. Shahraeini, M.H. Javidi, and M.S. Ghazizadeh. Comparison between communication infrastructures of centralized and decentralized wide area measurement systems. IEEE Trans. on Smart Grid, 2(1):206-211, 2011.

[69] N. Honda M. Murakami and J. Nishino. A study on the modeling ability of the ids method: A soft computing technique using patternbased information processing. Int. Journal of Approximate Reasoning, 45(3):470-487, 2007.

[70] F. Doostmohammadi, A. Esmaili, S. H H Sadeghi, A. Nasiri, and H.A. Talebi. A model predictive method for efficient power ramp rate control of wind turbines. In IECON 2012 - 38th Annual Conference on IEEE Industrial Electronics Society, pages 881-885, 2012.

[71] M. Murakami, M. Murakami, and N. Honda. Performance of the ids method as a soft computing tool. Fuzzy Systems, IEEE Transactions on, 16(6):15821596, 2008. ISSN 1063-6706. doi: 10.1109/TFUZZ.2008.2005693. 
[72] Mohammad Jawad Ghorbani, M. Akhbari, and H. Mokhtari. Direct torque control of induction motor by active learning method. In Power Electronic Drive Systems Technologies Conference (PEDSTC), 2010 1st, pages 267-272, 2010. doi: 10.1109/PEDSTC.2010.5471817.

[73] Y. Sakurai, N. Honda, and J. Nishino. Acquisition of control knowledge of nonholonomic system by active learning method. In Systems, Man and Cybernetics, 2003. IEEE International Conference on, volume 3, pages 24002405 vol.3, 2003. doi: 10.1109/ICSMC.2003.1244243.

[74] S. Haykin. Neural networks and leaning machines. In Prentice Hall, 3rd Edition, 1998. doi: 10.1109/PEDSTC.2010.5471817.

[75] N. Honda M. Murakami. Recursive fuzzy modeling based on fuzzy interpolation. Journal of Advanced Computational Intelligence and Intelligent Informatics, 3(2):114-125, 1999.

[76] Sridhar Chouhan, Jawad Ghorbani, Hakan Inan, Ali Feliachi, and Muhammad A. Choudhry. Smart mas restoration for distribution system with microgrids. In IEEE Power and Energy Society General Meeting (PES), pages 1-5, 2013. doi: 10.1109/PESMG.2013.6672945.

[77] J. Ghorbani, S. Chouhan, M.A. Choudhry, and A. Feliachi. Hybrid multi agent approach for power distribution system restoration. In Energytech, 2013 IEEE, pages 1-5, 2013.

[78] M. Pipattanasomporn, H. Feroze, and S. Rahman. Multi-agent systems in a distributed smart grid: Design and implementation. In Power Systems Conference and Exposition, 2009. PSCE '09. IEEE/PES, pages 1-8, 2009.

[79] SEL 735 Power Quality and Revenue Meter, Instruction Manual. Schweitzer Engineering Laboratories, Inc., Pullman, WA, 2011-2013.

[80] Nuvo 1000 Series, User's Manual. Neousys Technology Inc., 2011. 